\title{
Searches for Gravitational Waves from Known Pulsars at Two Harmonics in 2015-2017 LIGO Data
}

B. P. Abbott ${ }^{1}$, R. Abbott ${ }^{1}$, T. D. Abbott ${ }^{2}$, S. Abraham ${ }^{3}$, F. Acernese ${ }^{4,5}$, K. Ackley $^{6}$, C. Adams $^{7}$, R. X. Adhikari ${ }^{1}$, V. B. Adya ${ }^{8,9}$, C. Affeldt ${ }^{8,9}$, M. Agathos ${ }^{10}$, K. Agatsuma ${ }^{11}$, N. Aggarwal ${ }^{12}$, O. D. Aguiar ${ }^{13}$, L. Aiello ${ }^{14,15}$, A. Ain ${ }^{3}$, P. Ajith ${ }^{16}$, G. Allen ${ }^{17}$ A. Allocca ${ }^{18,19}$, M. A. Aloy $^{20}$, P. A. Altin ${ }^{21}$, A. Amato ${ }^{22}$, A. Ananyeva ${ }^{1}$, S. B. Anderson ${ }^{1}$, W. G. Anderson ${ }^{23}$, S. V. Angelova ${ }^{24}$, S. Antier ${ }^{25}$, S. Appert ${ }^{1}$, K. Arai ${ }^{1}$, M. C. Araya ${ }^{1}$, J. S. Areeda ${ }^{26}$, M. Arène ${ }^{27}$, N. Arnaud ${ }^{25,28}$, S. Ascenzi ${ }^{29,30}$, G. Ashton ${ }^{6}$, S. M. Aston ${ }^{7}$, P. Astone ${ }^{31}$, F. Aubin ${ }^{32}$, P. Aufmuth ${ }^{9}$, K. AultONeal ${ }^{33}$, C. Austin ${ }^{2}$, V. Avendano ${ }^{34}$, A. Avila-Alvarez ${ }^{26}$, S. Babak ${ }^{27,35}$, P. Bacon ${ }^{27}$, F. Badaracco ${ }^{14,15}$, M. K. M. Bader ${ }^{36}$, S. Bae ${ }^{37}$, M. Bailes ${ }^{38}$, P. T. Baker ${ }^{39}$, F. Baldaccini ${ }^{40,41}$ G. Ballardin ${ }^{28}$, S. W. Ballmer ${ }^{42}$, S. Banagiri ${ }^{43}$, J. C. Barayoga ${ }^{1}$, S. E. Barclay ${ }^{44}$, B. C. Barish ${ }^{1}$, D. Barker ${ }^{45}$, K. Barkett $^{46}$, S. Barnum ${ }^{12}$, F. Barone ${ }^{4,5}$, B. Barr ${ }^{44}$, L. Barsotti ${ }^{12}$, M. Barsuglia ${ }^{27}$, D. Barta ${ }^{47}$, J. Bartlett ${ }^{45}$, I. Bartos ${ }^{48}$, R. Bassiri ${ }^{49}$, A. Basti ${ }^{18,19}$, M. Bawaj ${ }^{41,50}$, J. C. Bayley ${ }^{44}$, M. Bazzan ${ }^{51,52}$, B. Bécsy ${ }^{53}$, M. Bejger ${ }^{27,54}$, I. Belahcene ${ }^{25}$, A. S. Bell ${ }^{44}$, D. Beniwal ${ }^{55}$, B. K. Berger ${ }^{49}$, G. Bergmann ${ }^{8,9}$, S. Bernuzzi ${ }^{56,57}$, J. J. Bero ${ }^{58}$, C. P. L. Berry ${ }^{59}$, D. Bersanetti ${ }^{60}$, A. Bertolini ${ }^{36}$, J. Betzwieser ${ }^{7}$, R. Bhandare ${ }^{61}$, J. Bidler ${ }^{26}$, I. A. Bilenko ${ }^{62}$, S. A. Bilgili ${ }^{39}$, G. Billingsley ${ }^{1}$, J. Birch ${ }^{7}$, R. Birney ${ }^{24}$, O. Birnholtz ${ }^{58}$, S. Biscans $s^{1,12}$, S. Biscoveanu ${ }^{6}$, A. Bisht ${ }^{9}$, M. Bitossi ${ }^{19,28}$, M. A. Bizouard ${ }^{25}$, J. K. Blackburn ${ }^{1}$, C. D. Blair ${ }^{7}$, D. G. Blair ${ }^{63}$, R. M. Blair ${ }^{45}$, S. Bloemen ${ }^{64}$, N. Bode ${ }^{8,9}$, M. Boer ${ }^{65}$, Y. Boetzel ${ }^{66}$, G. Bogaert ${ }^{65}$, F. Bondu ${ }^{67}$, E. Bonilla ${ }^{49}$, R. Bonnand ${ }^{32}$, P. Booker ${ }^{8,9}$, B. A. Boom ${ }^{36}$, C. D. Booth ${ }^{68}$, R. Bork ${ }^{1}$, V. Boschi ${ }^{28}$, S. Bose ${ }^{3,69}$, K. Bossie ${ }^{7}$, V. Bossilkov ${ }^{63}$, J. Bosveld ${ }^{63}$, Y. Bouffanais ${ }^{27}$, A. Bozzi ${ }^{28}$, C. Bradaschia ${ }^{19}$, P. R. Brady ${ }^{23}$, A. Bramley ${ }^{7}$, M. Branchesi ${ }^{14,15}$, J. E. Brau ${ }^{70}$, T. Briant ${ }^{71}$, J. H. Briggs ${ }^{44}$, F. Brighenti ${ }^{72,73}$, A. Brillet ${ }^{65}$, M. Brinkmann ${ }^{8,9}$, V. Brisson ${ }^{25,191}$, P. Brockill ${ }^{23}$, A. F. Brooks ${ }^{1}$, D. D. Brown ${ }^{55}$, S. Brunett ${ }^{1}$, A. Buikema ${ }^{12}$, T. Bulik ${ }^{74}$, H. J. Bulten ${ }^{36,75}$, A. Buonanno ${ }^{35,76}$, D. Buskulic ${ }^{32}$, C. Buy ${ }^{27}$, R. L. Byer ${ }^{49}$, M. Cabero ${ }^{8,9}$, L. Cadonati ${ }^{77}$, G. Cagnoli ${ }^{22,78}$, C. Cahillane ${ }^{1}$, J. Calderón Bustillo ${ }^{6}$, T. A. Callister ${ }^{1}$, E. Calloni ${ }^{5,79}$, J. B. Camp ${ }^{80}$, W. A. Campbell ${ }^{6}$,

M. Canepa ${ }^{6,81}$, K. C. Cannon ${ }^{82}$, H. Cao ${ }^{55}$, J. Cao ${ }^{83}$, E. Capocasa ${ }^{27}$, F. Carbognani ${ }^{28}$, S. Caride ${ }^{84}$, M. F. Carney ${ }^{59}$, G. Carullo ${ }^{18}$, J. Casanueva Diaz ${ }^{19}$, C. Casentini2 ${ }^{29,30}$, S. Caudill ${ }^{36}$, M. Cavaglià ${ }^{85}$, F. Cavalier ${ }^{25}$, R. Cavalieri ${ }^{28}$, G. Cella ${ }^{19}$, P. Cerdá-Durán ${ }^{20}$, G. Cerretani $^{18,19}$, E. Cesarini ${ }^{30,86}$, O. Chaibi ${ }^{65}$, K. Chakravarti ${ }^{3}$, S. J. Chamberlin ${ }^{87}$, M. Chan ${ }^{44}$, S. Chao ${ }^{88}$, P. Charlton ${ }^{89}$,

E. A. Chase ${ }^{59}$, E. Chassande-Mottin ${ }^{27}$, D. Chatterjee ${ }^{23}$, M. Chaturvedi ${ }^{61}$, B. D. Cheeseboro ${ }^{39}$, H. Y. Chen ${ }^{90}$, X. Chen ${ }^{63}$, Y. Chen $^{46}$, H.-P. Cheng ${ }^{48}$, C. K. Cheong ${ }^{91}$, H. Y. Chia ${ }^{48}$, A. Chincarini ${ }^{60}$, A. Chiummo ${ }^{28}$, G. Cho ${ }^{92}$, H. S. Cho ${ }^{93}$, M. Cho ${ }^{76}$,

N. Christensen ${ }^{65,94}$, Q. Chu ${ }^{63}$, S. Chua ${ }^{71}$, K. W. Chung ${ }^{91}$, S. Chung ${ }^{63}$, G. Ciani ${ }^{51,52}$, A. A. Ciobanu ${ }^{55}$, R. Ciolfi ${ }^{95,96}$, F. Cipriano ${ }^{65}$, A. Cirone ${ }^{60,81}$, F. Clara ${ }^{45}$, J. A. Clark ${ }^{77}$, P. Clearwater ${ }^{97}$, F. Cleva ${ }^{65}$, C. Cocchieri ${ }^{85}$, E. Coccia ${ }^{14,15}$, P.-F. Cohadon ${ }^{71}$, D. Cohen ${ }^{25}$, R. Colgan ${ }^{98}$, M. Colleoni ${ }^{99}$, C. G. Collette ${ }^{100}$, C. Collins ${ }^{11}$, L. R. Cominsky ${ }^{101}$, M. Constancio, Jr. ${ }^{13}$, L. Conti ${ }^{52}$, S. J. Cooper ${ }^{11}$, P. Corban ${ }^{7}$, T. R. Corbitt ${ }^{2}$, I. Cordero-Carrión ${ }^{102}$, K. R. Corley ${ }^{98}$, N. Cornish ${ }^{53}$, A. Corsi ${ }^{84}$, S. Cortese $^{28}$, C. A. Costa ${ }^{13}$, R. Cotesta $^{35}$,

M. W. Coughlin ${ }^{1}$, S. B. Coughlin ${ }^{59,68}$, J.-P. Coulon ${ }^{65}$, S. T. Countryman ${ }^{98}$, P. Couvares ${ }^{1}$, P. B. Covas ${ }^{99}$, E. E. Cowan ${ }^{77}$,

D. M. Coward ${ }^{63}$, M. J. Cowart ${ }^{7}$, D. C. Coyne ${ }^{1}$, R. Coyne ${ }^{103}$, J. D. E. Creighton ${ }^{23}$, T. D. Creighton ${ }^{104}$, J. Cripe ${ }^{2}$, M. Croquette ${ }^{71}$, S. G. Crowder ${ }^{105}$, T. J. Cullen ${ }^{2}$, A. Cumming ${ }^{44}$, L. Cunningham ${ }^{44}$, E. Cuoco ${ }^{28}$, T. Dal Canton ${ }^{80}$, G. Dálya ${ }^{106}$, S. L. Danilishin ${ }^{8,9}$, S. D’Antonio ${ }^{30}$, K. Danzmann ${ }^{8,9}$, A. Dasgupta ${ }^{107}$, C. F. Da Silva Costa ${ }^{48}$, L. E. H. Datrier ${ }^{44}$, V. Dattilo ${ }^{28}$, I. Dave ${ }^{61}$, M. Davier ${ }^{25}$, D. Davis ${ }^{42}$, E. J. Daw ${ }^{108}$, D. DeBra ${ }^{49}$, M. Deenadayalan ${ }^{3}$, J. Degallaix ${ }^{22}$, M. De Laurentis ${ }^{5,79}$, S. Deléglise ${ }^{71}$, W. Del Pozzo ${ }^{18,19^{\prime}}$, L. M. DeMarchi ${ }^{59}$, N. Demos ${ }^{12}$, T. Dent ${ }^{8,9,109}$, R. De Pietri ${ }^{57,10}$, J. Derby ${ }^{26}$, R. De Rosa ${ }^{5,79}$, C. De Rossi ${ }^{22,28}$, R. DeSalvo ${ }^{111}$, O. de Varona ${ }^{8,9}$, S. Dhurandhar ${ }^{3}$, M. C. Díaz ${ }^{104}$, T. Dietrich ${ }^{36}$, L. Di Fiore ${ }^{5}$, M. Di Giovanni ${ }^{96,112}$, T. Di Girolamo ${ }^{5,79}$, A. Di Lieto ${ }^{18,19}$, B. Ding ${ }^{100}$, S. Di Pace ${ }^{31,113}$, I. Di Palma ${ }^{31,113}$, F. Di Renzo ${ }^{18,19}$, A. Dmitriev ${ }^{11}$, Z. Doctor ${ }^{90}$, F. Donovan ${ }^{12}$, K. L. Dooley ${ }^{6,85}$, S. Doravari ${ }^{8,9}$, I. Dorrington ${ }^{68}$, T. P. Downes ${ }^{23}$, M. Drago ${ }^{14,15}$, J. C. Driggers ${ }^{45}$, Z. Du ${ }^{83}$, J.-G. Ducoin ${ }^{25}$, P. Dupej ${ }^{44}$, S. E. Dwyer ${ }^{45}$, P. J. Easter ${ }^{6}$, T. B. Edo ${ }^{108}$, M. C. Edwards ${ }^{94}$, A. Effler ${ }^{7}$, P. Ehrens ${ }^{1}$, J. Eichholz ${ }^{1}$, S. S. Eikenberry ${ }^{48}$, M. Eisenmann ${ }^{32}$, R. A. Eisenstein ${ }^{12}$, R. C. Essick ${ }^{90}$, H. Estelles ${ }^{99}$, D. Estevez ${ }^{32}$, Z. B. Etienne ${ }^{39}$, T. Etzel ${ }^{1}$, M. Evans ${ }^{12}$,

T. M. Evans ${ }^{7}$, V. Fafone ${ }^{14,29,30}$, H. Fair ${ }^{42}$, S. Fairhurst ${ }^{68}$, X. Fan ${ }^{83}$, S. Farinon ${ }^{60}$, B. Farr ${ }^{70}$, W. M. Farr ${ }^{11}$, E. J. Fauchon-Jones ${ }^{68}$, M. Favata ${ }^{34}$, M. Fays ${ }^{108}$, M. Fazio ${ }^{114}$, C. Fee ${ }^{115}$, J. Feicht ${ }^{1}$, M. M. Fejer ${ }^{49}$, F. Feng ${ }^{27}$, A. Fernandez-Galiana ${ }^{2}$, I. Ferrante ${ }^{18,19}$,

E. C. Ferreira ${ }^{13}$, T. A. Ferreira ${ }^{13}$, F. Ferrini ${ }^{28}$, F. Fidecaro ${ }^{18,19}$, I. Fiori ${ }^{28}$, D. Fiorucci' ${ }^{27}$, M. Fishbach ${ }^{90}$, R. P. Fisher ${ }^{42,116}$,

J. M. Fishner ${ }^{12}$, M. Fitz-Axen ${ }^{43}$, R. Flaminio ${ }^{32,117}$, M. Fletcher ${ }^{44}$, E. Flynn ${ }^{26}$, H. Fong ${ }^{118}$, J. A. Font ${ }^{20,119}$, P. W. F. Forsyth ${ }^{21}$, J.-D. Fournier ${ }^{65}$, S. Frasca ${ }^{31,113}$, F. Frasconi ${ }^{19}{ }^{3}$, Z. Frei ${ }^{106}$, A. Freise ${ }^{11}$, R. Frey ${ }^{70}$, V. Frey ${ }^{25}$, P. Fritschel ${ }^{12}$, V. V. Frolov ${ }^{7}$, P. Fulda ${ }^{48}$, M. Fyffe ${ }^{7}$, H. A. Gabbard ${ }^{44}$, B. U. Gadre ${ }^{3}$, S. M. Gaebel' ${ }^{11}$, J. R. Gair ${ }^{120}$, L. Gammaitoni ${ }^{40}$, M. R. Ganija ${ }^{55}$, S. G. Gaonkar ${ }^{3}$, A. Garcia ${ }^{26}$, C. García-Quirós ${ }^{99}$, F. Garufi ${ }^{5,79}$, B. Gateley ${ }^{45}$, S. Gaudio ${ }^{33}$, G. Gaur ${ }^{121}$, V. Gayathri ${ }^{122}$, G. Gemme ${ }^{60}$, E. Genin $^{28}$, A. Gennai ${ }^{19}$, D. George ${ }^{17}$, J. George ${ }^{61}$, L. Gergely ${ }^{123}$, V. Germain ${ }^{32}$, S. Ghonge ${ }^{77}$, Abhirup Ghosh ${ }^{16}$, Archisman Ghosh ${ }^{36}$, S. Ghosh ${ }^{23}$, B. Giacomazzo ${ }^{96,112}$, J. A. Giaime ${ }^{2,7}$, K. D. Giardina ${ }^{7}$, A. Giazotto ${ }^{19,192}$, K. Gill ${ }^{33}$, G. Giordano ${ }^{4,5}$, L. Glover ${ }^{111}$, P. Godwin ${ }^{87}$, E. Goetz ${ }^{45}$, R. Goetz ${ }^{48}$, B. Goncharov ${ }^{6}$, G. González ${ }^{2}$, J. M. Gonzalez Castro ${ }^{18,19}$, A. Gopakumar ${ }^{124}$,

M. L. Gorodetsky ${ }^{62}$, S. E. Gossan ${ }^{1}$, M. Gosselin ${ }^{28}$, R. Gouaty ${ }^{32}$, A. Grado ${ }^{5,125}$, C. Graef ${ }^{44}$, M. Granata ${ }^{22}$, A. Grant ${ }^{44}$, S. Gras $^{12}$, P. Grassia ${ }^{1}$, C. Gray ${ }^{45}$, R. Gray ${ }^{44}$, G. Greco ${ }^{72,73}$, A. C. Green ${ }^{11,48}$, R. Green ${ }^{68}$, E. M. Gretarsson ${ }^{33}$, P. Groot ${ }^{64}$, H. Grote ${ }^{68}$, S. Grunewald ${ }^{35}$, P. Gruning ${ }^{25}$, G. M. Guidi ${ }^{72,73}$, H. K. Gulati ${ }^{107}$, Y. Guo ${ }^{36}$, A. Gupta ${ }^{87}$, M. K. Gupta ${ }^{107}$, E. K. Gustafson ${ }^{1}$, R. Gustafson ${ }^{126}$, L. Haegel ${ }^{99}$, O. Halim ${ }^{14,15}$, B. R. Hall ${ }^{69}$, E. D. Hall ${ }^{12}$, E. Z. Hamilton ${ }^{68}$, G. Hammond ${ }^{44}$, M. Haney ${ }^{66}$, 
M. M. Hanke ${ }^{8,9}$, J. Hanks ${ }^{45}$, C. Hanna ${ }^{87}$, M. D. Hannam ${ }^{68}$, O. A. Hannuksela ${ }^{91}$, J. Hanson ${ }^{7}$, T. Hardwick ${ }^{2}$, K. Haris ${ }^{16}$ J. Harms ${ }^{14,15}$, G. M. Harry ${ }^{127}$, I. W. Harry ${ }^{35}$, C.-J. Haster ${ }^{18}$, K. Haughian ${ }^{44}$, F. J. Hayes ${ }^{44}$, J. Healy ${ }^{58}$, A. Heidmann ${ }^{71}$, M. C. Heintze ${ }^{7}$, H. Heitmann ${ }^{65}$, P. Hello ${ }^{25}$, G. Hemming ${ }^{28}$, M. Hendry ${ }^{4}$, I. S. Heng ${ }^{44}$, J. Hennig ${ }^{8,9}$, A. W. Heptonstall ${ }^{1}$, Francisco Hernandez Vivanco ${ }^{6}$, M. Heurs ${ }^{8,9}$, S. Hild ${ }^{44}$, T. Hinderer ${ }^{36,128,129}$, W. C. G. Ho ${ }^{130}$, D. Hoak ${ }^{28}$, S. Hochheim ${ }^{8,9}$, D. Hofman ${ }^{22}$, A. M. Holgado ${ }^{17}$, N. A. Holland ${ }^{21}$, K. Holt ${ }^{7}$, D. E. Holz ${ }^{90}$, P. Hopkins ${ }^{68}$, C. Horst ${ }^{23}$, J. Hough ${ }^{44}$, E. J. Howell ${ }^{63}$, C. G. $\mathrm{Hoy}^{68}$, A. Hreibi ${ }^{65}$, E. A. Huerta ${ }^{17}$, D. Huet ${ }^{25}$, B. Hughey ${ }^{33}$, M. Hulko ${ }^{1}$, S. Husa ${ }^{99}$, S. H. Huttner ${ }^{44}$, T. Huynh-Dinh ${ }^{7}$, B. Idzkowski ${ }^{74}$, A. Iess ${ }^{29,30}$, C. Ingram ${ }^{55}$, R. Inta ${ }^{84}$, G. Intini ${ }^{31113}$, B. Irwin ${ }^{115}$, H. N. Isa ${ }^{44}$, J.-M. Isac ${ }^{71}$, M. Isi ${ }^{1}$, B. R. Iyer ${ }^{16}$, K. Izumi ${ }^{45}$, T. Jacqmin ${ }^{71}$, S. J. Jadhav ${ }^{131}$, K. Jani ${ }^{77}$, N. N. Janthalur ${ }^{131}$, P. Jaranowski ${ }^{132}$, A. C. Jenkins ${ }^{133}$, J. Jiang ${ }^{48}$,

D. S. Johnson ${ }^{17}$, A. W. Jones ${ }^{11}$, D. I. Jones ${ }^{134}$, R. Jones ${ }^{44}$, R. J. G. Jonker ${ }^{36}$, L. Ju ${ }^{63}$, J. Junker ${ }^{8,9}$, C. V. Kalaghatgi ${ }^{68}$, V. Kalogera ${ }^{59}$, B. Kamai ${ }^{1}$, S. Kandhasamy ${ }^{85}$, G. Kang ${ }^{37}$, J. B. Kanner ${ }^{1}$, S. J. Kapadia ${ }^{23}$, S. Karki ${ }^{70}$, K. S. Karvinen ${ }^{8,9}$, R. Kashyap ${ }^{16}$, M. Kasprzack ${ }^{1}$, S. Katsanevas ${ }^{28}$, E. Katsavounidis ${ }^{12}$, W. Katzman ${ }^{7}$, S. Kaufer ${ }^{9}$, K. Kawabe ${ }^{45}$, N. V. Keerthana ${ }^{3}$, F. Kéfélian ${ }^{65}$, D. Keitel ${ }^{44}$, R. Kennedy ${ }^{108}$, J. S. Key ${ }^{135}$, F. Y. Khalili ${ }^{62}$, H. Khan ${ }^{26}$, I. Khan ${ }^{14,30}$, S. Khan ${ }^{8,9}$, Z. Khan ${ }^{107}$, E. A. Khazanov ${ }^{136}$, M. Khursheed ${ }^{61}$, N. Kijbunchoo ${ }^{21}$, Chunglee Kim ${ }^{137}$, J. C. Kim ${ }^{138}$, K. Kim ${ }^{91}$, W. Kim ${ }^{55}$, W. S. Kim ${ }^{139}$, Y.-M. Kim ${ }^{140}$, C. Kimball ${ }^{59}$, E. J. King ${ }^{55}$, P. J. King ${ }^{45}$, M. Kinley-Hanlon ${ }^{127}$, R. Kirchhoff ${ }^{8,9}$, J. S. Kissel ${ }^{45}$, L. Kleybolte ${ }^{141}$, J. H. Klika ${ }^{23}$, S. Klimenko ${ }^{48}$, T. D. Knowles ${ }^{39}$, P. Koch ${ }^{8,9}$, S. M. Koehlenbeck ${ }^{8,9}$, G. Koekoek ${ }^{36,142}$, S. Koley ${ }^{36}$, V. Kondrashov ${ }^{1}$, A. Kontos ${ }^{12}$, N. Koper ${ }^{8,9}$, M. Korobko ${ }^{141}$, W. Z. Korth ${ }^{1}$, I. Kowalska ${ }^{74}$, D. B. Kozak ${ }^{1}$, V. Kringel ${ }^{8,9}$, N. Krishnendu ${ }^{143}$, A. Królak ${ }^{144,145}$, G. Kuehn ${ }^{8,9}$, A. Kumar ${ }^{131}$, P. Kumar ${ }^{146}$, R. Kumar ${ }^{107}$, S. Kumar' ${ }^{16}$, L. Kuo ${ }^{88}$, A. Kutynia ${ }^{144}$, S. Kwang ${ }^{23}$, B. D. Lackey ${ }^{35}$, K. H. Lai ${ }^{91}$, T. L. Lam ${ }^{91}$, M. Landry ${ }^{45}$, B. B. Lane ${ }^{12}$, R. N. Lang ${ }^{147}$, J. Lange ${ }^{58}$, B. Lantz ${ }^{49}$, R. K. Lanza ${ }^{12}$, A. Lartaux-Vollard ${ }^{25}$, P. D. Lasky ${ }^{6}$, M. Laxen ${ }^{7}$, A. Lazzarini ${ }^{1}$, C. Lazzaro ${ }^{52}$, P. Leaci ${ }^{31,113}$, S. Leavey,9, Y. K. Lecoeuche ${ }^{45}$, C. H. Lee ${ }^{93}$, H. K. Lee ${ }^{148^{\prime}}$, H. M. Lee ${ }^{149}$, H. W. Lee ${ }^{138}$, J. Lee ${ }^{92}$, K. Lee ${ }^{44}$, J. Lehmann ${ }^{8,9}$, A. Lenon ${ }^{39}$, N. Leroy ${ }^{25}$, N. Letendre ${ }^{32}$, Y. Levin ${ }^{6,98}$, J. Li $^{83}$,

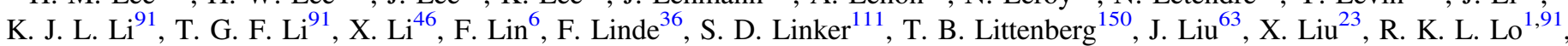
N. A. Lockerbie ${ }^{24}$, L. T. London ${ }^{68}$, A. Longo ${ }^{151,152}$, M. Lorenzini ${ }^{14,15}$, V. Loriette ${ }^{153}$, M. Lormand ${ }^{7}$, G. Losurdo ${ }^{19}$, J. D. Lough ${ }^{8,9}$ ',

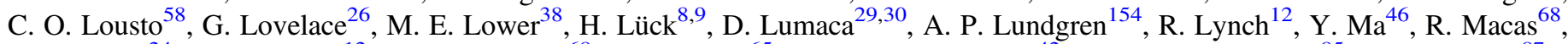
S. Macfoy $^{24}$, M. MacInnis ${ }^{12}$, D. M. Macleod ${ }^{68}$, A. Macquet ${ }^{65}$, F. Magaña-Sandoval ${ }^{42}$, L. Magaña Zertuche ${ }^{85}$, R. M. Magee ${ }^{87}$, E. Majorana ${ }^{31}$, I. Maksimovic ${ }^{153}$, A. Malik ${ }^{61}$, N. Man ${ }^{65}$, V. Mandic ${ }^{43}$, V. Mangano ${ }^{44}$, G. L. Mansell ${ }^{12,45}$, M. Manske ${ }^{21,23}$, M. Mantovani ${ }^{28}$, F. Marchesoni ${ }^{41,50}$, F. Marion ${ }^{32}$, S. Márka ${ }^{98}$, Z. Márka ${ }^{98}$, C. Markakis ${ }^{10,17}$, A. S. Markosyan ${ }^{49}$, A. Markowitz ${ }^{1}$, E. Maros ${ }^{1}$, A. Marquina ${ }^{102}$, S. Marsat ${ }^{35}$, F. Martelli ${ }^{72,73}$, I. W. Martin ${ }^{44}$, R. M. Martin 34 , D. V. Martynov ${ }^{11}$, K. Mason ${ }^{12}$, E. Massera $^{108}$, A. Masserot ${ }^{32}$, T. J. Massinger ${ }^{1}$, M. Masso-Reid ${ }^{44}$, S. Mastrogiovanni ${ }^{31,113}$, A. Matas ${ }^{35,43}$, F. Matichard ${ }^{1,12}$, L. Matone ${ }^{98}$, N. Mavalvala ${ }^{12}$, N. Mazumder ${ }^{69}$, J. J. McCann ${ }^{63}$, R. McCarthy ${ }^{45}$, D. E. McClelland ${ }^{21}$, S. McCormick ${ }^{7}$, L. McCuller ${ }^{12}$, S. C. McGuire ${ }^{155}$, J. McIver ${ }^{1}$, D. J. McManus ${ }^{21}$, T. McRae ${ }^{21}$, S. T. McWilliams ${ }^{39}$, D. Meacher ${ }^{87}$, G. D. Meadors ${ }^{6}$, M. Mehmet ${ }^{8,9}$, A. K. Mehta ${ }^{16}$, J. Meidam ${ }^{36}$, A. Melatos ${ }^{97}$, G. Mendell ${ }^{45}$, R. A. Mercer ${ }^{23}$, L. Mereni ${ }^{22}$, E. L. Merilh ${ }^{45}$, M. Merzougui ${ }^{65}$, S. Meshkov ${ }^{1}$, C. Messenger ${ }^{44}$, C. Messick ${ }^{87}$, R. Metzdorff ${ }^{71}$, P. M. Meyers ${ }^{97}$, H. Miao ${ }^{11}$, C. Michel ${ }^{22}$, H. Middleton ${ }^{97}$, E. E. Mikhailov ${ }^{156}$, L. Milano ${ }^{5,79}$, A. L. Miller ${ }^{48}$, A. Miller ${ }^{31,113}$, M. Millhouse ${ }^{53}$, J. C. Mills ${ }^{68}$, M. C. Milovich-Goff ${ }^{111}$, O. Minazzoli ${ }^{65,157}$, Y. Minenkov ${ }^{30}$, A. Mishkin ${ }^{48}$, C. Mishra ${ }^{158}$, T. Mistry ${ }^{108}$, S. Mitra ${ }^{3}$, V. P. Mitrofanov ${ }^{62}$, G. Mitselmakher ${ }^{48}$, R. Mittleman ${ }^{12}$, G. Mo ${ }^{94}$, D. Moffa ${ }^{115}$, K. Mogushi ${ }^{85}$, S. R. P. Mohapatra ${ }^{12}$, M. Montani ${ }^{72,73}$, C. J. Moore ${ }^{10}$, D. Moraru ${ }^{45}$, G. Moreno $^{45}$, S. Morisaki ${ }^{82}$, B. Mours ${ }^{32}$, C. M. Mow-Lowry ${ }^{11}$, Arunava Mukherjee ${ }^{8,9}$, D. Mukherjee ${ }^{23}$, S. Mukherjee ${ }^{104}$, N. Mukund ${ }^{3}$, A. Mullavey ${ }^{7}$, J. Munch ${ }^{55}$, E. A. Muñiz ${ }^{42}$, M. Muratore ${ }^{33}$, P. G. Murray ${ }^{44}$, A. Nagar ${ }^{86,159,160}$, I. Nardecchia ${ }^{29,30}$, L. Naticchioni ${ }^{31,113}$, R. K. Nayak ${ }^{161}$, J. Neilson ${ }^{111}$, G. Nelemans ${ }^{36,64}$, T. J. N. Nelson ${ }^{7}$, M. Nery ${ }^{8,9}$, A. Neunzert ${ }^{126}$, K. Y. Ng $^{12}$, S. $\mathrm{Ng}^{55}$, P. Nguyen ${ }^{70}$, D. Nichols ${ }^{36,128}$, S. Nissanke ${ }^{36,128}$, F. Nocera ${ }^{28}$, C. North $^{68}$, L. K. Nuttall ${ }^{154}$, M. Obergaulinger ${ }^{20}$, J. Oberling ${ }^{45}$, B. D. O’Brien ${ }^{48}$, G. D. O’Dea ${ }^{111}$, G. H. Ogin ${ }^{162}$, J. J. Oh ${ }^{139}$, S. H. Oh ${ }^{139}$, F. Ohme ${ }^{8,9}$, H. Ohta ${ }^{82}$, M. A. Okada ${ }^{13}$, M. Oliver ${ }^{99}$, P. Oppermann ${ }^{8,9}$, Richard J. Oram ${ }^{7}$, B. O'Reilly ${ }^{7}$, R. G. Ormiston ${ }^{43}$, L. F. Ortega ${ }^{48}$, R. O'Shaughnessy ${ }^{58}$,

S. Ossokine ${ }^{35}$, D. J. Ottaway ${ }^{55}$, H. Overmier ${ }^{7}$, B. J. Owen ${ }^{84}$, A. E. Pace ${ }^{87}$, G. Pagano ${ }^{18,19}$, M. A. Page ${ }^{63}$, A. Pai ${ }^{122}$, S. A. Pai ${ }^{61}$, J. R. Palamos ${ }^{70}$, O. Palashov ${ }^{136}$, C. Palomba ${ }^{31}$, A. Pal-Singh ${ }^{141}$, Huang-Wei Pan ${ }^{88}$, B. Pang ${ }^{46}$, P. T. H. Pang ${ }^{91}$, C. Pankow ${ }^{59}$, F. Pannarale ${ }^{31,113}$, B. C. Pant ${ }^{61}$, F. Paoletti ${ }^{19}$, A. Paoli ${ }^{28}$, A. Parida ${ }^{3}$, W. Parker ${ }^{7,155}$, D. Pascucci ${ }^{44}$, A. Pasqualetti ${ }^{28}$,

R. Passaquieti ${ }^{18,19}$, D. Passuello ${ }^{19}$, M. Patil ${ }^{145}$, B. Patricelli ${ }^{18,19}$, B. L. Pearlstone ${ }^{44}$, C. Pedersen ${ }^{68}$, M. Pedraza ${ }^{1}$, R. Pedurand ${ }^{22,163}$, A. Pele ${ }^{7}$, S. Penn ${ }^{164}$, C. J. Perez ${ }^{45}$, A. Perreca ${ }^{96,112}$, H. P. Pfeiffer ${ }^{35,118}$, M. Phelps ${ }^{8,9}$, K. S. Phukon ${ }^{3}$, O. J. Piccinni ${ }^{31,113}$, M. Pichot ${ }^{65}$, F. Piergiovanni ${ }^{72,73}$, G. Pillant ${ }^{28}$, L. Pinard ${ }^{22}$, M. Pirello ${ }^{45}$, M. Pitkin ${ }^{44}$, R. Poggiani ${ }^{18,19}$, D. Y. T. Pong ${ }^{91}$,

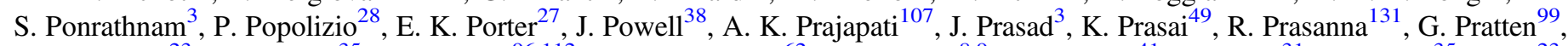
T. Prestegard ${ }^{23}$, S. Privitera ${ }^{35}$, G. A. Prodi ${ }^{96,112}$, L. G. Prokhorov ${ }^{62}$, O. Puncken ${ }^{8,9}$, M. Punturo ${ }^{41}$, P. Puppo ${ }^{31}$, M. Pürrer $^{35}$, H. Qi $^{23}$, V. Quetschke ${ }^{104}$, P. J. Quinonez ${ }^{33}$, E. A. Quintero ${ }^{1}$, R. Quitzow-James ${ }^{70}$, F. J. Raab ${ }^{45}$, H. Radkins ${ }^{45}$, N. Radulescu ${ }^{65}$, P. Raffai ${ }^{106}$, S. Raja ${ }^{61}$, C. Rajan ${ }^{61}$, B. Rajbhandari ${ }^{84}$, M. Rakhmanov ${ }^{104}$, K. E. Ramirez ${ }^{104}$, A. Ramos-Buades ${ }^{99}$, Javed Rana ${ }^{3}$, K. Rao ${ }^{59}$, P. Rapagnani ${ }^{31,113}$, V. Raymond ${ }^{68}$, M. Razzano ${ }^{18,19}$, J. Read ${ }^{26}$, T. Regimbau ${ }^{32}$, L. Rei $^{60}$, S. Reid $^{24}$, D. H. Reitze ${ }^{1,48}$, W. Ren ${ }^{17}$, F. Ricci ${ }^{31,113}$, C. J. Richardson ${ }^{33}$, J. W. Richardson ${ }^{1}$, P. M. Ricker ${ }^{17}$, K. Riles ${ }^{126}$, M. Rizzo ${ }^{59}$, N. A. Robertson ${ }^{1,44}$, R. Robie ${ }^{44}$, F. Robinet ${ }^{25}$, A. Rocchi ${ }^{30}$, L. Rolland ${ }^{32}$, J. G. Rollinss ${ }^{1}$, V. J. Roma ${ }^{70}$, M. Romanelli ${ }^{67}$, R. Romano ${ }^{4,5}$, C. L. Romel ${ }^{45}$, J. H. Romie ${ }^{7}$, K. Rose $^{115}$, D. Rosińska ${ }^{54,165}$, S. G. Rosofsky ${ }^{17}$, M. P. Ross $^{166}$, S. Rowan $^{44}$, A. Rüdiger ${ }^{8,9,193}$, P. Ruggi ${ }^{28}$, G. Rutins ${ }^{167}$, K. Ryan $^{45}$, S. Sachdev ${ }^{1}$, T. Sadecki ${ }^{45}$, M. Sakellariadou ${ }^{133}$, L. Salconi ${ }^{28}$, M. Saleem ${ }^{143}$, A. Samajdar ${ }^{36}$, L. Sammut ${ }^{6}$, E. J. Sanchez ${ }^{1}$, L. E. Sanchez ${ }^{1}$, N. Sanchis-Gual ${ }^{20}$, V. Sandberg ${ }^{45}$, J. R. Sanders ${ }^{42}$, K. A. Santiago ${ }^{34}$, N. Sarin ${ }^{6}$, B. Sassolas ${ }^{22}$, P. R. Saulson ${ }^{42}$, 
O. Sauter ${ }^{126}$, R. L. Savage ${ }^{45}$, P. Schale ${ }^{70}$, M. Scheel ${ }^{46}$, J. Scheuer ${ }^{59}$, P. Schmidt ${ }^{64}$, R. Schnabel ${ }^{141}$, R. M. S. Schofield ${ }^{70}$, A. Schönbeck ${ }^{141}$, E. Schreiber ${ }^{8,9}$, B. W. Schulte ${ }^{8,9}$, B. F. Schutz ${ }^{68}$, S. G. Schwalbe ${ }^{33}$, J. Scott ${ }^{44}$, S. M. Scott ${ }^{21}$, E. Seidel ${ }^{17}$, D. Sellers ${ }^{7}$, A. S. Sengupta ${ }^{168}$, N. Sennett ${ }^{35}$, D. Sentenac ${ }^{28}$, V. Sequino ${ }^{14,29,30}$, A. Sergeev ${ }^{136}$, Y. Setyawati ${ }^{8,9}$, D. A. Shaddock ${ }^{21}$, T. Shaffer ${ }^{45}$, M. S. Shahriar ${ }^{59}$, M. B. Shaner ${ }^{11}$, L. Shao ${ }^{35}$, P. Sharma ${ }^{61}$, P. Shawhan ${ }^{76}$, H. Shen ${ }^{17}$, R. Shink ${ }^{169}$, D. H. Shoemaker ${ }^{12}$, D. M. Shoemaker ${ }^{77}$, S. ShyamSundar ${ }^{61}$, K. Siellez ${ }^{77}$, M. Sieniawska ${ }^{54}$, D. Sigg ${ }^{45}$, A. D. Silva ${ }^{13}$, L. P. Singer ${ }^{80}$, N. Singh $^{74}$, A. Singhal ${ }^{14,31}$, A. M. Sintes ${ }^{99}$, S. Sitmukhambetov ${ }^{104}$, V. Skliris ${ }^{68}$, B. J. J. Slagmolen ${ }^{21}$, T. J. Slaven-Blair ${ }^{63}$, J. R. Smith ${ }^{26}$, R. J. E. Smith ${ }^{6}$, S. Somala ${ }^{170}$, E. J. Son ${ }^{139}$, B. Sorazu ${ }^{44}$, F. Sorrentino ${ }^{60}$, T. Souradeep ${ }^{3}$, E. Sowell ${ }^{84}$, A. P. Spencer ${ }^{44}$, A. K. Srivastava ${ }^{107}$, V. Srivastava ${ }^{42}$, K. Staats ${ }^{59}$, C. Stachie $^{65}$, M. Standke ${ }^{8,9}$, D. A. Steer ${ }^{27}$, M. Steinke ${ }^{8,9}$, J. Steinlechner ${ }^{44,141}$, S. Steinlechner ${ }^{141}$, D. Steinmeyer ${ }^{8,9}$, S. P. Stevenson ${ }^{38}$, D. Stocks ${ }^{49}$, R. Stone ${ }^{104}$, D. J. Stops ${ }^{11}$, K. A. Strain ${ }^{44}$, G. Stratta ${ }^{72,73}$, S. E. Strigin ${ }^{62}$, A. Strunk ${ }^{45}$, R. Sturani ${ }^{171}$, A. L. Stuver ${ }^{172}$, V. Sudhir ${ }^{12}$, T. Z. Summerscales ${ }^{173}$, L. Sun ${ }^{1}$, S. Sunil ${ }^{107}$, J. Suresh $^{3}$, P. J. Sutton ${ }^{68}$, B. L. Swinkels ${ }^{36}$, M. J. Szczepańczyk ${ }^{33}$, M. Tacca ${ }^{36}$, S. C. Tait ${ }^{44}$, C. Talbot ${ }^{6}$, D. Talukder ${ }^{70}$, D. B. Tanner ${ }^{48}$, M. Tápai ${ }^{123}$, A. Taracchini ${ }^{35}$, J. D. Tasson ${ }^{94}$, R. Taylor ${ }^{1}$, F. Thies ${ }^{8,9}$, M. Thomas ${ }^{7}$, P. Thomas ${ }^{45}$, S. R. Thondapu ${ }^{61}$, K. A. Thorne ${ }^{7}$, E. Thrane ${ }^{6}$, Shubhanshu Tiwari ${ }^{96,112}$, Srishti Tiwari ${ }^{124}$, V. Tiwari ${ }^{68}$, K. Toland $^{44}$, M. Tonelli ${ }^{18,19}$, Z. Tornasi $^{44}$, A. Torres-Forné ${ }^{174}$, C. I. Torrie ${ }^{1}$, D. Töyrä ${ }^{11}$, F. Travasso ${ }^{28,41}$, G. Traylor ${ }^{7}$, M. C. Tringali ${ }^{74}$, A. Trovato ${ }^{27}$, L. Trozzo ${ }^{19,175}$, R. Trudeau ${ }^{1}$, K. W. Tsang ${ }^{36}$, M. Tse ${ }^{12}$, R. Tso ${ }^{46}$, L. Tsukada ${ }^{82}$, D. Tsuna ${ }^{82}$, D. Tuyenbayev ${ }^{104}$, K. Ueno ${ }^{82}$, D. Ugolini ${ }^{176}$, C. S. Unnikrishnan ${ }^{124}$, A. L. Urban ${ }^{2}$, S. A. Usman ${ }^{68}$, H. Vahlbruch ${ }^{9}$, G. Vajente ${ }^{1}$, G. Valdes $^{2}$, N. van Bakel ${ }^{36}$, M. van Beuzekom ${ }^{36}$, J. F. J. van den Brand ${ }^{36,75}$,

C. Van Den Broeck ${ }^{36,177}$, D. C. Vander-Hyde ${ }^{42}$, J. V. van Heijningen ${ }^{63}$, L. van der Schaaf ${ }^{36}$, A. A. van Veggel ${ }^{44}$, M. $^{2}$ Vardaro $^{51,52}$, V. Varma ${ }^{46}$, S. Vass ${ }^{1}$, M. Vasúth ${ }^{47}$, A. Vecchio ${ }^{11}$, G. Vedovato ${ }^{52}$, J. Veitch ${ }^{44}$, P. J. Veitch ${ }^{55}$, K. Venkateswara ${ }^{166}$, G. Venugopalan ${ }^{1}$, D. Verkindt ${ }^{32}$, F. Vetrano ${ }^{72,73}$, A. Viceré ${ }^{72,73}$, A. D. Viets ${ }^{23}$, D. J. Vine ${ }^{167}$, J.-Y. Vinet $^{65}$, S. Vitale $^{12}$, T. Vo ${ }^{42}$, H. Vocca $^{40,41}$, C. Vorvick ${ }^{45}$, S. P. Vyatchanin ${ }^{62}$, A. R. Wade ${ }^{1}$, L. E. Wade ${ }^{115}$, M. Wade ${ }^{115}$, R. Walet ${ }^{36}$, M. Walker ${ }^{26}$, L. Wallace ${ }^{1}$, S. Walsh $^{23}$, G. Wang ${ }^{14,19}$, H. Wang ${ }^{11}$, J. Z. Wang ${ }^{126}$, W. H. Wang ${ }^{104}$, Y. F. Wang ${ }^{91}$, R. L. Ward ${ }^{21}$, Z. A. Warden ${ }^{33}$, J. Warner $^{45}$, M. Was $^{32}$, J. Watchi ${ }^{100}$, B. Weaver ${ }^{45}$, L.-W. Wei ${ }^{8,9}$, M. Weinert ${ }^{8,9}$, A. J. Weinstein ${ }^{1}$, R. Weiss ${ }^{12}$, F. Wellmann ${ }^{8,9}$, L. Wen $^{63}$, E. K. Wessel ${ }^{17}$, P. Weßels ${ }^{8,9}$, J. W. Westhouse ${ }^{33}$, K. Wette ${ }^{21}$, J. T. Whelan ${ }^{58}$, B. F. Whiting ${ }^{48}$, C. Whittle ${ }^{12}$, D. M. Wilken ${ }^{8,9}$, D. Williams ${ }^{44}$, A. R. Williamson ${ }^{36,128}$, J. L. Willis ${ }^{1}$, B. Willke ${ }^{8,9}$, M. H. Wimmer ${ }^{8,9}$, W. Winkler ${ }^{8,9}$, C. C. Wipf ${ }^{1}$, H. Wittel ${ }^{8,9}$, G. Woan ${ }^{44}$, J. Woehler ${ }^{8,9}$, J. K. Wofford ${ }^{58}$, J. Worden ${ }^{45}$, J. L. Wright ${ }^{44}$, D. S. Wu ${ }^{8,9}$, D. M. Wysocki ${ }^{58}$, L. Xiao ${ }^{1}$, H. Yamamoto ${ }^{1}$,

C. C. Yancey ${ }^{76}$, L. Yang ${ }^{114}$, M. J. Yapp ${ }^{21}$, M. Yazback ${ }^{48}$, D. W. Yeeles ${ }^{68}$, Hang Yu ${ }^{12}$, Haocun Yu ${ }^{12}$, S. H. R. Yuen ${ }^{91}$, M. Yvert ${ }^{32}$, A. K. Zadrożny ${ }^{104,144}$, M. Zanolin ${ }^{33}$, T. Zelenova ${ }^{28}$, J.-P. Zendri ${ }^{52}$, M. Zevin ${ }^{59}$, J. Zhang ${ }^{63}$, L. Zhang ${ }^{1}$, T. Zhang ${ }^{44}$, C. Zhao ${ }^{63}$, M. Zhou ${ }^{59}$, Z. Zhou ${ }^{59}$, X. J. Zhu ${ }^{6}$, M. E. Zucker ${ }^{1,12}$, J. Zweizig ${ }^{1}$

The LIGO Scientific Collaboration and the Virgo Collaboration,

Z. Arzoumanian ${ }^{178}$, S. Bogdanov ${ }^{179}$, I. Cognard ${ }^{180,181}$, A. Corongiu ${ }^{182}$, T. Enoto $^{183}$, P. Freire ${ }^{184}$, K. C. Gendreau ${ }^{178}$

L. Guillemot ${ }^{180,181}$, A. K. Harding ${ }^{185}$, F. Jankowski ${ }^{186}$, M. J. Keith ${ }^{186}$, M. Kerr ${ }^{187}$, A. Lyne ${ }^{186}$, J. Palfreyman ${ }^{188}$, A. Possenti ${ }^{182,189}$ A. Ridolfi ${ }^{184}$, B. Stappers ${ }^{186}$, G. Theureau ${ }^{180,181,190}$, and P. Weltervrede ${ }^{186}$

${ }^{1}$ LIGO, California Institute of Technology, Pasadena, CA 91125, USA; 1sc-spokesperson@ligo.org, virgo-spokesperson@ego-gw.it ${ }^{2}$ Louisiana State University, Baton Rouge, LA 70803, USA

${ }^{3}$ Inter-University Centre for Astronomy and Astrophysics, Pune 411007, India ${ }^{4}$ Università di Salerno, Fisciano, I-84084 Salerno, Italy

${ }^{5}$ INFN, Sezione di Napoli, Complesso Universitario di Monte S.Angelo, I-80126 Napoli, Italy

${ }^{6}$ OzGrav, School of Physics \& Astronomy, Monash University, Clayton 3800, Victoria, Australia ${ }^{7}$ LIGO Livingston Observatory, Livingston, LA 70754, USA

${ }^{8}$ Max Planck Institute for Gravitational Physics (Albert Einstein Institute), D-30167 Hannover, Germany

${ }^{9}$ Leibniz Universität Hannover, D-30167 Hannover, Germany

${ }^{10}$ University of Cambridge, Cambridge CB2 1TN, UK

${ }^{11}$ University of Birmingham, Birmingham B15 2TT, UK

${ }^{12}$ LIGO, Massachusetts Institute of Technology, Cambridge, MA 02139, USA

${ }^{13}$ Instituto Nacional de Pesquisas Espaciais, 12227-010 São José dos Campos, São Paulo, Brazil

${ }^{14}$ Gran Sasso Science Institute (GSSI), I-67100 L'Aquila, Italy

${ }^{15}$ INFN, Laboratori Nazionali del Gran Sasso, I-67100 Assergi, Italy

${ }^{16}$ International Centre for Theoretical Sciences, Tata Institute of Fundamental Research, Bengaluru 560089, India

${ }^{17}$ NCSA, University of Illinois at Urbana-Champaign, Urbana, IL 61801, USA ${ }^{18}$ Università di Pisa, I-56127 Pisa, Italy

${ }^{19}$ INFN, Sezione di Pisa, I-56127 Pisa, Italy

${ }^{20}$ Departamento de Astronomía y Astrofísica, Universitat de València, E-46100 Burjassot, València, Spain

${ }^{21}$ OzGrav, Australian National University, Canberra, Australian Capital Territory 0200, Australia

${ }^{22}$ Laboratoire des Matériaux Avancés (LMA), CNRS/IN2P3, F-69622 Villeurbanne, France

${ }^{23}$ University of Wisconsin-Milwaukee, Milwaukee, WI 53201, USA

${ }^{24}$ SUPA, University of Strathclyde, Glasgow G1 1XQ, UK

${ }^{25}$ LAL, Univ. Paris-Sud, CNRS/IN2P3, Université Paris-Saclay, F-91898 Orsay, France

${ }^{26}$ California State University Fullerton, Fullerton, CA 92831, USA

${ }^{27}$ APC, AstroParticule et Cosmologie, Université Paris Diderot, CNRS/IN2P3, CEA/Irfu, Observatoire de Paris, Sorbonne Paris Cité, F-75205 Paris Cedex 13, France

${ }^{28}$ European Gravitational Observatory (EGO), I-56021 Cascina, Pisa, Italy

${ }^{29}$ Università di Roma Tor Vergata, I-00133 Roma, Italy

${ }^{30}$ INFN, Sezione di Roma Tor Vergata, I-00133 Roma, Italy

${ }^{31}$ INFN, Sezione di Roma, I-00185 Roma, Italy

${ }^{32}$ Laboratoire d'Annecy de Physique des Particules (LAPP), Univ. Grenoble Alpes, Université Savoie Mont Blanc, CNRS/IN2P3, F-74941 Annecy, France

${ }^{33}$ Embry-Riddle Aeronautical University, Prescott, AZ 86301, USA 


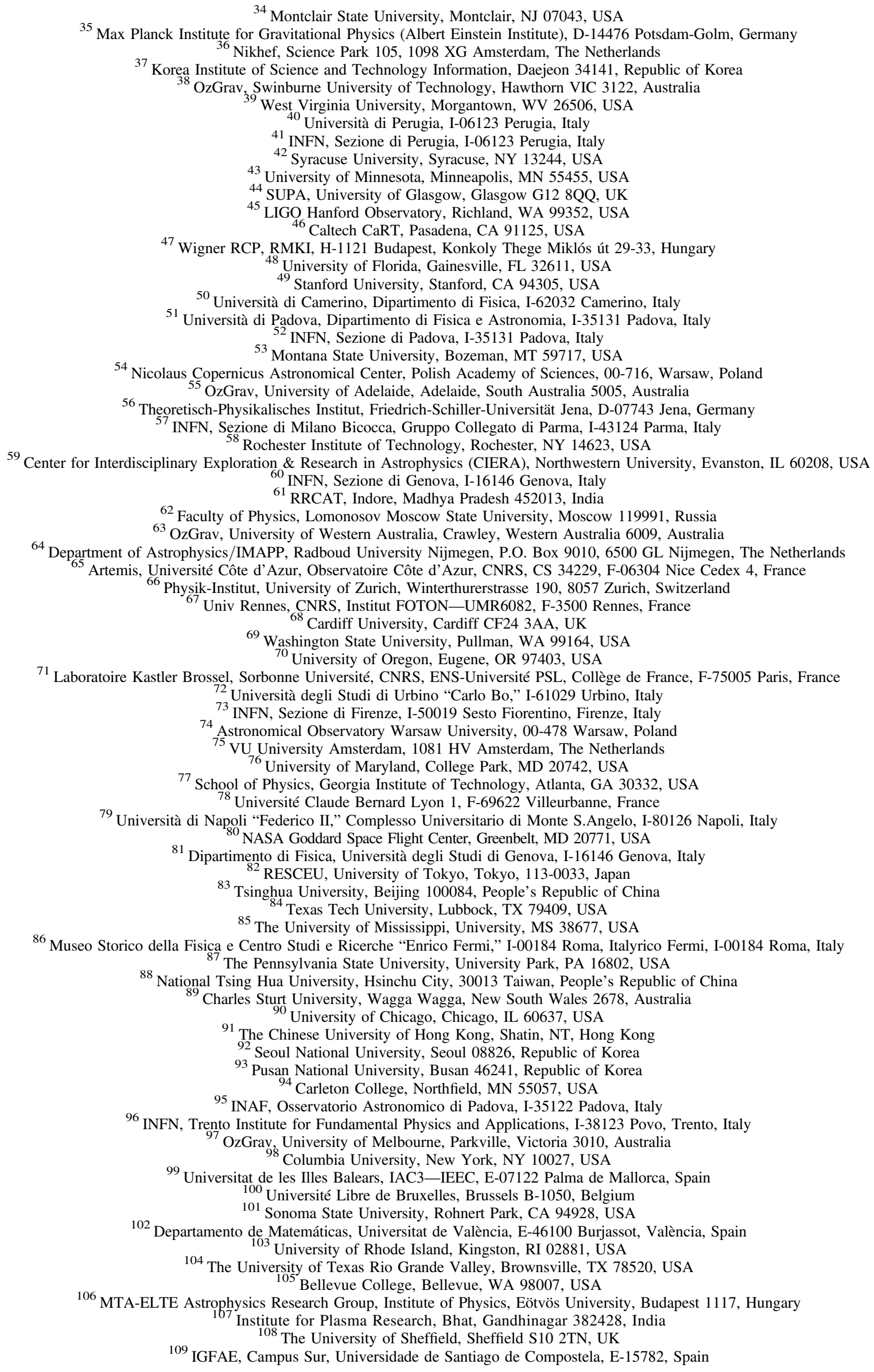




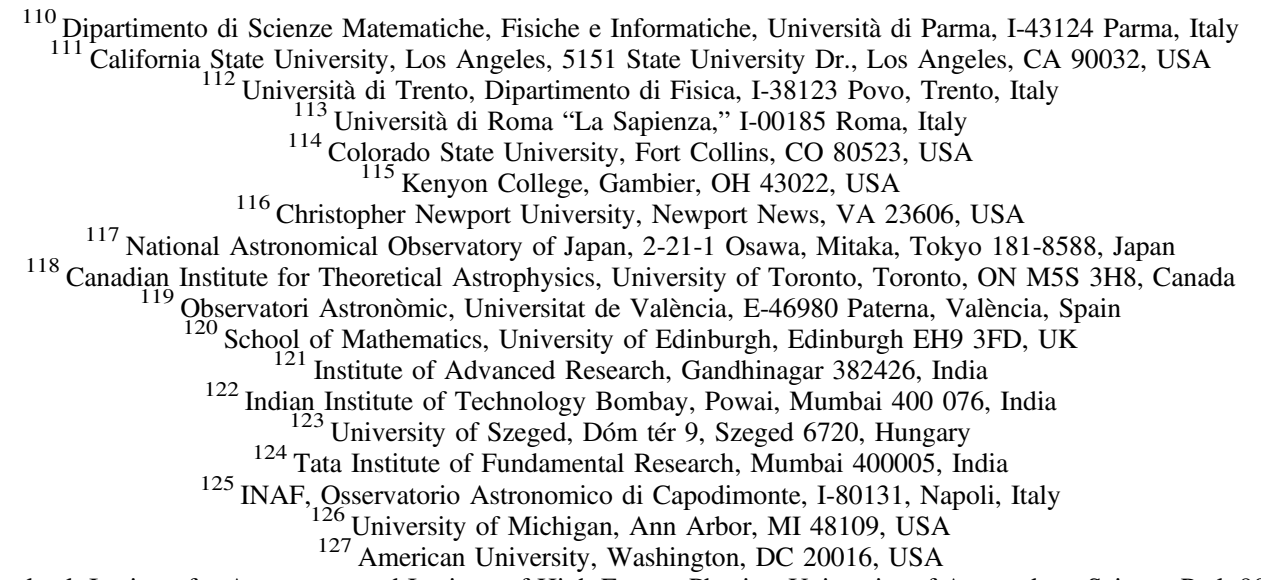

${ }^{128}$ GRAPPA, Anton Pannekoek Institute for Astronomy and Institute of High-Energy Physics, University of Amsterdam, Science Park 904, 1098 XH Amsterdam, The Netherlands

${ }^{129}$ Delta Institute for Theoretical Physics, Science Park 904, 1090 GL Amsterdam, The Netherlands

${ }^{130}$ Department of Physics and Astronomy, Haverford College, 370 Lancaster Avenue, Haverford, PA 19041, USA

${ }^{131}$ Directorate of Construction, Services \& Estate Management, Mumbai 400094, India

${ }^{132}$ University of Białystok, 15-424 Białystok, Poland

${ }^{133}$ King's College London, University of London, London WC2R 2LS, UK

${ }^{134}$ University of Southampton, Southampton SO17 1BJ, UK

${ }^{135}$ University of Washington Bothell, Bothell, WA 98011, USA

${ }^{136}$ Institute of Applied Physics, Nizhny Novgorod, 603950, Russia

${ }^{137}$ Ewha Womans University, Seoul 03760, Republic of Korea

${ }^{138}$ Inje University Gimhae, South Gyeongsang 50834, Republic of Korea

${ }^{139}$ National Institute for Mathematical Sciences, Daejeon 34047, Republic of Korea

${ }^{140}$ Ulsan National Institute of Science and Technology, Ulsan 44919, Republic of Korea

${ }^{141}$ Universität Hamburg, D-22761 Hamburg, Germany

${ }^{142}$ Maastricht University, P.O. Box 616, 6200 MD Maastricht, The Netherlands

${ }^{143}$ Chennai Mathematical Institute, Chennai 603103, India

${ }^{144}$ NCBJ, 05-400 Świerk-Otwock, Poland

${ }^{145}$ Institute of Mathematics, Polish Academy of Sciences, 00656 Warsaw, Poland

${ }_{146}$ Cornell University, Ithaca, NY 14850 , USA

${ }^{147}$ Hillsdale College, Hillsdale, MI 49242, USA

${ }^{148}$ Hanyang University, Seoul 04763 , Republic of Korea

${ }^{149}$ Korea Astronomy and Space Science Institute, Daejeon 34055, Republic of Korea

${ }^{150}$ NASA Marshall Space Flight Center, Huntsville, AL 35811, USA

${ }^{151}$ Dipartimento di Matematica e Fisica, Università degli Studi Roma Tre, I-00146 Roma, Italy

${ }^{152}$ INFN, Sezione di Roma Tre, I-00146 Roma, Italy ${ }_{153}$ ESPCI, CNRS, F-75005 Paris, France

${ }^{154}$ University of Portsmouth, Portsmouth, PO1 3FX, UK

${ }^{155}$ Southern University and A\&M College, Baton Rouge, LA 70813, USA

${ }^{156}$ College of William and Mary, Williamsburg, VA 23187, USA

${ }^{157}$ Centre Scientifique de Monaco, 8 quai Antoine Ier, MC-98000, Monaco

${ }_{159}^{158}$ Indian Institute of Technology Madras, Chennai 600036, India

${ }^{159}$ INFN Sezione di Torino, Via P. Giuria 1, I-10125 Torino, Italy

${ }^{160}$ Institut des Hautes Etudes Scientifiques, F-91440 Bures-sur-Yvette, France

${ }^{162}{ }^{161}$ IISER-Kolkata, Mohanpur, West Bengal 741252, India

${ }^{162}$ Whitman College, 345 Boyer Avenue, Walla Walla, WA 99362, USA

${ }^{163}$ Université de Lyon, F-69361 Lyon, France

${ }^{164}$ Hobart and William Smith Colleges, Geneva, NY 14456, USA

165 Janusz Gil Institute of Astronomy, University of Zielona Góra, 65-265 Zielona Góra, Poland

${ }^{166}$ University of Washington, Seattle, WA 98195, USA

${ }^{167}$ SUPA, University of the West of Scotland, Paisley PA1 2BE, UK

${ }^{168}$ Indian Institute of Technology, Gandhinagar Ahmedabad Gujarat 382424, India

${ }_{169}$ Université de Montréal/Polytechnique, Montreal, QC H3T 1J4, Canada

${ }^{170}$ Indian Institute of Technology Hyderabad, Sangareddy, Khandi, Telangana 502285, India

${ }^{171}$ International Institute of Physics, Universidade Federal do Rio Grande do Norte, Natal RN 59078-970, Brazil

${ }^{172}$ Villanova University, 800 Lancaster Ave., Villanova, PA 19085, USA

173 Andrews University, Berrien Springs, MI 49104, USA

${ }^{174}$ Max Planck Institute for Gravitationalphysik (Albert Einstein Institute), D-14476 Potsdam-Golm, Germany

${ }^{175}$ Università di Siena, I-53100 Siena, Italy

${ }^{176}$ Trinity University, San Antonio, TX 78212, USA

${ }^{177}$ Van Swinderen Institute for Particle Physics and Gravity, University of Groningen, Nijenborgh 4, 9747 AG Groningen, The Netherlands

${ }^{178}$ X-Ray Astrophysics Laboratory, NASA Goddard Space Flight Center, Greenbelt, MD 20771, USA

${ }^{179}$ Columbia Astrophysics Laboratory, Columbia University, 550 West 120th Street, New York, NY, 10027, USA

${ }^{180}$ Laboratoire de Physique et Chimie de l'Environnement et de l'Espace-Université d'Orléans/CNRS, F-45071 Orléans Cedex 02, France

${ }^{81}$ Station de Radioastronomie de Nançay, Observatoire de Paris, CNRS/INSU, F-18330 Nançay, France

${ }^{182}$ INAF-Osservatorio Astronomico di Cagliari, via della Scienza 5, I-09047 Selargius, Italy

${ }^{183}$ Hakubi Center for Advanced Research and Department of Astronomy, Kyoto University, Kyoto 606-8302, Japan

${ }^{184}$ Max-Planck-Institut für Radioastronomie, Auf dem Hügel 69, D-53121 Bonn, Germany 


\author{
${ }^{185}$ Astrophysics Science Division, NASA Goddard Space Flight Center, Greenbelt, MD 20771, USA \\ 186 Jodrell Bank Centre for Astrophysics, School of Physics and Astronomy, University of Manchester, Manchester, M13 9PL, UK \\ ${ }^{187}$ Space Science Division, Naval Research Laboratory, Washington, DC 20375-5352, USA \\ ${ }^{188}$ Department of Physical Sciences, University of Tasmania, Private Bag 37, Hobart, Tasmania 7001, Australia \\ ${ }^{189}$ Università di Cagliari, Dipartimento di Fisica, I-09042, Monserrato, Italy \\ ${ }^{190}$ LUTH, Observatoire de Paris, PSL Research University, CNRS, Université Paris Diderot, Sorbonne Paris Cité, F-92195 Meudon, France \\ Received 2019 March 18; revised 2019 May 7; accepted 2019 May 8; published 2019 June 26
}

\begin{abstract}
We present a search for gravitational waves from 222 pulsars with rotation frequencies $\gtrsim 10 \mathrm{~Hz}$. We use advanced LIGO data from its first and second observing runs spanning 2015-2017, which provides the highest-sensitivity gravitational-wave data so far obtained. In this search we target emission from both the $l=m=2$ mass quadrupole mode, with a frequency at twice that of the pulsar's rotation, and the $l=2, m=1$ mode, with a frequency at the pulsar rotation frequency. The search finds no evidence for gravitational-wave emission from any pulsar at either frequency. For the $l=m=2$ mode search, we provide updated upper limits on the gravitationalwave amplitude, mass quadrupole moment, and fiducial ellipticity for 167 pulsars, and the first such limits for a further 55. For 20 young pulsars these results give limits that are below those inferred from the pulsars' spin-down. For the Crab and Vela pulsars our results constrain gravitational-wave emission to account for less than $0.017 \%$ and $0.18 \%$ of the spin-down luminosity, respectively. For the recycled millisecond pulsar J0711 -6830 our limits are only a factor of 1.3 above the spin-down limit, assuming the canonical value of $10^{38} \mathrm{~kg} \mathrm{~m}{ }^{2}$ for the star's moment of inertia, and imply a gravitational-wave-derived upper limit on the star's ellipticity of $1.2 \times 10^{-8}$. We also place new limits on the emission amplitude at the rotation frequency of the pulsars.
\end{abstract}

Key words: gravitational waves - pulsars: general - stars: neutron

Supporting material: machine-readable table

\section{Introduction}

There have been several previous searches for persistent (or continuous) quasi-monochromatic gravitational waves emitted by a selection of known pulsars using data from the LIGO, Virgo, and GEO600 gravitational-wave detectors (Abbott et al. 2004, 2005, 2017a, 2007, 2008, 2010; Abadie et al. 2011; Aasi et al. 2014). In the majority of these, the signals that have been searched for are those that would be expected from stars with a nonzero $l=m=2$ mass quadrupole moment $Q_{22}$ and with polarization content consistent with the expectations of general relativity (see, e.g., Zimmermann \& Szedenits 1979; Bonazzola \& Gourgoulhon 1996; Jaranowski et al. 1998). Such signals would be produced at twice the stellar rotation frequencies, and searches have generally assumed that the rotation frequency derived from electromagnetic observations of the pulsars is phase locked to the star's rotation and thus the gravitationalwave signal. Some searches have been performed where the assumption of the phase locking to the observed electromagnetic signal has been slightly relaxed, allowing the signal to be potentially offset over a small range of frequencies $(\sim 10-100 \mathrm{mHz})$ and first frequency derivatives (Abbott et al. 2008, 2017b; Aasi et al. 2015b). A search including the prospect of the signal's polarization content deviating from the purely tensorial modes predicted by general relativity has also been performed in Abbott et al. (2018a). None of these searches have detected a gravitational-wave signal from any of the pulsars that were targeted. Thus, stringent upper limits of the gravitational-wave amplitude, mass quadrupole moment, and ellipticity have been set.

Emission of gravitational waves at a pulsar's rotation frequency from the $l=2, m=1$ harmonic mode, in addition to emission at twice the rotation frequency from the

\footnotetext{
191 Deceased, 2018 February.

192 Deceased, 2017 November.

193 Deceased, 2018 July.
}

$l=m=2$ mode, has long been theorized (Zimmermann \& Szedenits 1979; Zimmermann 1980; Jones \& Andersson 2002). The fiducial emission mechanism would be from a biaxial or triaxial star undergoing free precession. In the case of a precessing biaxial star, or a precessing triaxial star with a small "wobble angle," the electromagnetic pulsar emission frequency would be modulated slightly, with the gravitational-wave emission being emitted at frequencies close to once and twice the time-averaged rotation frequency. There is only weak observational evidence for any pulsar showing precession (see the discussions in, e.g., Jones 2012; Durant et al. 2013, and references therein), and free precession would be quickly damped, but as shown in Jones (2010), the existence of a superfluid interior gives rise to the possibility for gravitational-wave emission at the rotation frequency even for a nonprecessing star. A search for emission at both once and twice the rotation frequency for 43 pulsars using data from LIGO's fifth science run has been performed in Pitkin et al. (2015). That analysis saw no evidence for signals at the rotation frequency and was consistent with the search conducted for signals purely from the $l=m=2$ mode (Abbott et al. 2010).

The searches implemented in this work are specifically designed for the case where the signal's phase evolution is very well known over the course of full gravitational-wave detector observing runs. Therefore, here we will only focus on the assumption that emission occurs at precisely once and twice the observed rotation frequency, as given by the model in Jones (2010), so we do not account for the possibility of any of the sources undergoing free precession.

Previous searches, combining the results given in Aasi et al. (2014) and Abbott et al. (2017a), have included a total of 271 pulsars. The most stringent upper limit on gravitationalwave amplitude from the $l=m=2$ mode was set for PSR J1918-0642 at $1.6 \times 10^{-26}$, and the most stringent upper limit on the fiducial ellipticity (see Appendix A, Equations (4) 
and (6)) was set for PSR J0636+5129 at $1.3 \times 10^{-8}$ (Abbott et al. 2017a). However, for these particular pulsars, both of which are millisecond pulsars (MSPs), the gravitationalwave amplitude limits are above the fiducial spin-down limit (see Appendix A and Equation (9)). In the search described in Abbott et al. (2017a), there were eight pulsars for which their observed gravitational-wave limits were below the fiducial spin-down limits, with the upper limits on emission from the Crab pulsar (PSR J0534+2200) and Vela pulsar (PSR J0835 -4510) being factors of more than 20 and 9 below their respective spin-down limits. ${ }^{194}$

Concurrently with this work, a search has been performed for 33 pulsars using advanced LIGO data from the second observing run in which the assumption of phase locking between the electromagnetically observed signal and gravitational-wave signal is relaxed by allowing the signal model to vary freely over a narrow band of frequencies and frequency derivatives (Abbott et al. 2019). Even with the slight sensitivity decrease compared to the analysis presented here, due to the wider parameter space, that analysis gives limits that are below the spin-down limit for 13 of the pulsars.

\subsection{Signal Model}

Using the formalism shown in Jones (2015) and Pitkin et al. (2015), the gravitational-wave waveform from the $l=2$, $m=1$ harmonic mode can be written as

$$
\begin{array}{r}
h_{21}(t)=-\frac{C_{21}}{2}\left[F_{+}^{D}(\alpha, \delta, \psi ; t) \sin \iota \cos \iota \cos (\Phi(t)\right. \\
\left.\left.+\Phi_{21}^{C}\right)+F_{\times}^{D}(\alpha, \delta, \psi ; t) \sin \iota \sin \left(\Phi(t)+\Phi_{21}^{C}\right)\right],
\end{array}
$$

and that from the $l=m=2$ mode can be written as

$$
\begin{aligned}
& h_{22}(t)=-C_{22}\left[F_{+}^{D}(\alpha, \delta, \psi ; t)\left(1+\cos ^{2} \iota\right) \cos (2 \Phi(t)\right. \\
& \left.\left.\quad+\Phi_{22}^{C}\right)+2 F_{\times}^{D}(\alpha, \delta, \psi ; t) \cos \iota \sin \left(2 \Phi(t)+\Phi_{22}^{C}\right)\right] .
\end{aligned}
$$

Here $C_{21}$ and $C_{22}$ represent the amplitudes of the components, $\Phi_{21}^{C}$ and $\Phi_{22}^{C}$ represent initial phases at a particular epoch, $\Phi(t)$ is the rotational phase of the source, and $\iota$ is the inclination of the source's rotation axis with respect to the line of sight. ${ }^{195}$ The detected amplitude is modulated by the detector response functions for the two polarizations of the signal (" + " and " $\times$ "), $F_{+}^{D}(\alpha, \delta, \psi ; t)$ and $F_{\times}^{D}(\alpha, \delta, \psi ; t)$, which depend on the location and orientation of detector $D$, the location of the source on the sky, defined by the R.A. $\alpha$ and decl. $\delta$, and the polarization angle of the source $\psi$.

As shown in Jones (2015), the waveforms given in Equations (1) and (2) describe a generic signal, but the amplitudes $\left(C_{21}\right.$ and $\left.C_{22}\right)$ and phases $\left(\Phi_{21}^{C}\right.$ and $\left.\Phi_{22}^{C}\right)$ can be related to intrinsic physical parameters describing a variety of source models, e.g., a triaxial star spinning about a principal axis (Abbott et al. 2004), a biaxial precessing star (Jones \& Andersson 2002), or a triaxial star not spinning about a principal axis (Jones 2010). In the standard case adopted for previous gravitational-wave searches of a triaxial star spinning about a principal axis, there is only emission at twice the rotation frequency from the $l=m=2$ mode, so only

\footnotetext{
194 In previous work we have often referred to observed gravitational-wave limits "surpassing," or "beating," the spin-down limits, which just means to say that the limits are lower than the equivalent spin-down limits.

195 For precessing stars the phase evolution $\Phi(t)$ in Equations (1) and (2) will not necessarily be given by the rotational phase, but it can differ by the precession frequency.
}

Equation (2) is nonzero. In this case the $C_{22}$ amplitude can be simply related to the standard gravitational-wave strain amplitude $h_{0}$ via $h_{0}=2 C_{22}{ }^{196}$ We can simply define the phase $\Phi_{22}^{C}$ as relating to the initial rotational phase $\phi_{0}$ via $\Phi_{22}^{C}=2 \phi_{0}$, noting that $\phi_{0}$ actually incorporates the sum of two phase parameters (an initial gravitational-wave phase and another phase offset) that are entirely degenerate and therefore not separately distinguishable (Jones 2015).

Despite Equations (1) and (2) not providing the intrinsic parameters of the source, they do break strong degeneracies between them, which are otherwise impossible to disentangle (see Pitkin et al. 2015, showing this for the case of a triaxial source not rotating about a principal axis).

In this work we adopt two analyses. The first assumes the standard picture of a triaxial star rotating around a principal axis from which we can simply relate the waveform amplitude $C_{22}$ to the gravitational-wave amplitude. In this case we can then compare this to the standard spin-down limit and can calculate each source's mass quadrupole $Q_{22}$ and fiducial ellipticity upper limits (see Appendix A for definitions of these standard quantities.) The second assumes the model of a triaxial star not spinning about a principal axis, for which there could be emission at both once or twice the rotation frequency. In this case we do not attempt to relate the signal amplitudes to any physical parameter of the source.

\subsection{Signal Strength}

For the $l=m=2$ quadrupole mode the strength of the emission is defined by the size of the mass quadrupole moment $Q_{22}$ (see Equations (3) and (5)), which is proportional to the ellipticity of the star and to the star's moment of inertia, and will therefore depend on the star's mass and also on the equation of state of neutron star matter (see, e.g., Ushomirsky et al. 2000; Owen 2005; Johnson-McDaniel \& Owen 2013). This ellipticity could be provided by some physical distortion of the star's crust or irregularities in the density profile of the star. For our purposes the mechanism providing the distortion must be sustained over long periods, e.g., the crust must be strong enough for any (submillimeter high) mountain to be maintained (see Owen 2005; Johnson-McDaniel \& Owen 2013, for discussions of the maximum sustainable ellipticities for various neutron star equations of state), or there must be a persistent strong internal magnetic field (e.g., Bonazzola \& Gourgoulhon 1996; Cutler 2002). Johnson-McDaniel \& Owen (2013) suggest that, assuming a standard set of neutron star equations of state, maximum fiducial ellipticities of a few $\times$ $10^{-6}$ could be sustained. Constraints on the neutron star equation of state are now starting to be probed using gravitational-wave observations from the binary neutron star coalescence observed as GW170817 (Abbott et al. 2017 c, 2018b). These constraints suggest that softer equations of state are favored over stiffer ones, which would imply smaller maximum crustal quadrupoles. An additional caveat to this is that the maximum crustal deformation is also dependent on the star's mass, and less massive stars would allow larger deformations (Horowitz 2010; Johnson-McDaniel \& Owen 2013), so there is still a wide range of uncertainty. Recent work on the strength of neutron star crusts consisting of

\footnotetext{
196 To maintain the sign convention between Equation (2) and the equivalent equation in, e.g., Jaranowski et al. (1998), the transform between $h_{0}$ and $C_{22}$ should more strictly be $h_{0}=-2 C_{22}$
} 
nuclear pasta suggests that these could have larger breaking strains and thus support larger ellipticities (Caplan et al. 2018).

It has recently been suggested by Woan et al. (2018) that the distribution of MSPs in the period-period derivative plane provides some observational evidence that they may all have a limiting minimum ellipticity of $\sim 10^{-9}$. This could be due to some common process that takes place during the recycling accretion stage that spins the pulsar up to millisecond periods. For example, there could be external magnetic field burial (see, e.g., Melatos \& Phinney 2001; Payne \& Melatos 2004), for which the size of the buried field is roughly the same across all stars, or similar levels of spin-up leading to crust breaking (e.g., Fattoyev et al. 2018). If this is true, it provides a compelling reason to look for emission from these objects.

For the model emitting at both $l=2, m=1,2$ modes, and assuming no precession, the signal amplitudes are related to combinations of moment-of-inertia asymmetries and orientation angles between the crust and core of the star (Jones 2010). These are related in a complex way to the $C_{21}$ and $C_{22}$ amplitudes given in Equations (1) and (2) (see Jones 2015). In general, if the $Q_{21}$ and $Q_{22}$ mass moments are equal, then the gravitational-wave strain from the $l=2, m=1$ mode would be roughly four times smaller owing to the fact that it is related to the square of the frequency and that mode is at half the frequency of the $l=m=2$ mode. However, we do not have good estimates of what the actual relative mass moments might be.

Note that one can in principle also obtain limits on a neutron star's deformation if one interprets some features of its timing properties as due to free precession. In this case, the limits involve a combination of the differences between the three principal moments of inertia, together with an angular parameter ("wobble angle") giving the amplitude of the precession. This can be done either for stars that show some periodic structure in their timing properties (see, e.g., Akgün et al. 2006; Ashton et al. 2017) or by assuming that some component of pulsar timing noise is due to precession (Cordes 1993). Note, however, that it is by no means clear whether pulsar timing really does provide evidence for free precession (Jones et al. 2017; Stairs et al. 2019).

\subsection{Search Methods}

As with the previous searches for gravitational waves from known pulsars described in Aasi et al. (2014) and Abbott et al. (2017a), we make use of three semi-independent search methods. We will not describe these methods in detail here, but we refer the reader to Aasi et al. (2014) for more information. Briefly, the three methods are as follows: a search using narrowband time-domain data to perform Bayesian parameter estimation for the unknown signal parameters, and marginal likelihood evaluation, for each pulsar (Dupuis \& Woan 2005; Pitkin et al. 2017); a search using the same narrowband time series, but Fourier-transformed into the frequency domain, to calculate the $\mathcal{F}$-statistic (Jaranowski et al. 1998) (or equivalent $\mathcal{G}$-statistic for constrained orientations; Jaranowski \& Królak 2010), with a frequentist-based amplitude upper limit estimation procedure (Feldman \& Cousins 1998); and a search in the frequency domain that makes use of splitting of any astrophysical signal into five frequency harmonics through the sidereal amplitude modulation given by the detector responses (Astone et al. 2010, 2012). The narrowband time-domain data are produced by heterodyning the raw detector strain data using the expected signal's phase evolution (Dupuis \& Woan 2005). It is then lowpass-filtered with a knee frequency of $0.25 \mathrm{~Hz}$ and downsampled, via averaging, creating a complex time series with one sample per minute, i.e., a bandwidth of $1 / 60 \mathrm{~Hz}$ centered about the expected signal frequency that is now at $0 \mathrm{~Hz}$. We call these approaches the Bayesian, $\mathcal{F}$ - $/ \mathcal{G}$-statistic, and $5 n$ vector methods, respectively. The first of these methods has been applied to all the pulsars in the sample (see Section 2.2), and again following Aasi et al. (2014) and Abbott et al. (2017a), at least two of the above methods have been applied to a selection of 34 high-value targets for which the observed limit is lower than, or closely approaches, the spin-down limit. The results of the 5n-vector analysis only use data from the LIGO O2 run (see Section 2.1).

All these methods have been adapted to deal with the potential for signals at both once and twice the rotation frequency. For the Bayesian method, when searching for such a signal the narrowband time series from both frequencies are included in a coherent manner, with common polarization angles $\psi$ and orientations $\iota$. For the $5 n$-vector and $\mathcal{F}$ - $/ \mathcal{G}$-statistic methods a simpler approach is taken, and signals at the two frequencies are searched for independently. The $\mathcal{F} / \mathcal{G}$-statistic approach for such a signal is described in more detail in Bejger \& Królak (2014). As a consequence, given that $C_{21}=0$ (see Equation (1)) corresponds to the case of a triaxial star rotating around one of its principal axes of inertia, results for the amplitude $C_{22}$ (Equation (2)) from the $5 n$-vector method are not given, as they are equivalent to those for the standard amplitude $h_{0}$.

In the case of a pulsar being observed to glitch during the run (see Section 2.2) the methods take different approaches. For the Bayesian method it is assumed that any glitch may produce an unknown offset between the electromagnetically observed rotational phase and the gravitational-wave phase. Therefore, an additional phase offset is added to the signal model at the time of the glitch, and this is included as a parameter to be estimated, while the gravitational-wave amplitude and orientation angles of the source (inclination and polarization) are assumed to remain fixed over the glitch. This is consistent with the analysis in Abbott et al. (2010), although it differs from the more recent analyses in Aasi et al. (2014) and Abbott et al. (2017a), in which each interglitch period was treated semiindependently, i.e., independent phases and polarization angles were assumed for each interglitch period, but two-dimensional marginalized posterior distributions on the gravitationalwave amplitude and cosine of the inclination angle from data before a glitch were used as a prior on those parameters when analyzing data after the glitch. For both the $\mathcal{F} / \mathcal{G}$-statistic and $5 n$-vector methods, as already done in Aasi et al. (2014) and Abbott et al. (2017a), each interglitch period is analyzed independently, i.e., no parameters are assumed to be coherent over the glitch, and the resulting statistics are incoherently combined.

The prior probability distributions for the unknown signal parameters, as used for the Bayesian and 5n-vector methods, are described in Appendix B.

The 5n-vector method uses a description of the gravitationalwave signal based on the concept of polarization ellipse. The relation of the amplitude parameter $H_{0}$ used by the $5 n$-vector method with both the standard strain amplitude $h_{0}$ and the $C_{21}$ amplitude given in Equation (1) is described in Appendix E. 


\section{Data}

In this section we briefly detail both the gravitationalwave data that have been used in the searches and the electromagnetic ephemerides for the selection of pulsars that have been included.

\subsection{Gravitational-wave Data}

The data analyzed in this paper consist of those obtained by the two LIGO detectors (the LIGO Hanford Observatory, commonly abbreviated to LHO or $\mathrm{H} 1$, and the LIGO Livingston Observatory, abbreviated to LLO or L1) taken during their first (Abbott et al. 2016) and second observing runs (O1 and $\mathrm{O} 2$, respectively) in their advanced detector configurations (Aasi et al. 2015a). ${ }^{197}$

Data from O1 between 2015 September 11 (with start times of 01:25:03 UTC and 18:29:03 UTC for LHO and LLO, respectively) and 2016 January 19 at 17:07:59 UTC have been used. The calibration of these data and the frequencydependent uncertainties on amplitude and phase over the run are described in detail in Cahillane et al. (2017). Over the course of the $\mathrm{O} 1$ run the calibration amplitude uncertainty was no larger than $5 \%$ and $10 \%$, and the phase uncertainty was no larger than $3^{\circ}$ and $4^{\circ}$, for LHO and LLO, respectively, over the frequency range $\sim 10-2000 \mathrm{~Hz}$ (these are derived from the $68 \%$ confidence levels given in Figure 11 of Cahillane et al. 2017). All data flagged as in "science mode," i.e., when the detectors were operating in a stable state, and for which the calibration was behaving as expected, have been used. This gave a total of 79 and 66 days of observing time for LHO and LLO, respectively, equivalent to duty factors of $60 \%$ and $51 \%$.

Data from O2 between 2016 November 30 at 16:00:00 UTC and 2017 August 25 at 22:00:00 UTC, for both LHO and LLO, have been used. An earlier version of the calibrated data for this observing run, as well as the uncertainty budget associated with it, is again described in Cahillane et al. (2017). However, data with an updated calibration have been produced and used in this analysis, with this having an improved uncertainty budget (Cahillane et al. 2018). Over the course of the $\mathrm{O} 2$ run the calibration amplitude uncertainty was no larger than $3 \%$ and $8 \%$ and the phase uncertainty was no larger than $3^{\circ}$ and $4^{\circ}$ for LHO and LLO, respectively, over the frequency range of $\sim 10-2000 \mathrm{~Hz}$. The data used in this analysis were post-processed to remove spurious jitter noise that affected detector sensitivity across a broad range of frequencies, particularly for data from LHO, and to remove some instrumental spectral lines (Davis et al. 2019; Driggers et al. 2019).

The Virgo gravitational-wave detector (Acernese et al. 2015) was operating during the last 25 days of O2 (Abbott et al. 2017d); however, due to its higher noise levels as compared to the LIGO detectors and the shorter observing time, Virgo data were not included in this analysis.

\subsection{Pulsars}

For this analysis we have gathered ephemerides for 222 pulsars based on radio, X-ray, and $\gamma$-ray observations. The observations have used the $42 \mathrm{ft}$ telescope and Lovell

\footnotetext{
197 The O1 and O2 data sets are publicly available via the Gravitational Wave Open Science Center at https://www.gw-openscience.org/O1 and https:// www.gw-openscience.org/O2, respectively (Vallisneri et al. 2015).
}

telescope at Jodrell Bank (UK), the Mount Pleasant Observatory $26 \mathrm{~m}$ telescope (Australia), the Parkes radio telescope (Australia), the Nançay Decimetric Radio Telescope (France), the Molonglo Observatory Synthesis Telescope (Australia), the Arecibo Observatory (Puerto Rico), the Fermi Large Area Telescope, and the Neutron Star Interior Composition Explorer (NICER). As with the search in Abbott et al. (2017a), the criterion for our selection of pulsars was that they have rotation frequencies greater than $10 \mathrm{~Hz}$, so that they are within the frequency band of greatest sensitivity of the LIGO instruments, and for which the calibration is well characterized. There are in fact three pulsars with rotation frequencies just below $10 \mathrm{~Hz}$ that we include (PSR J0117 +5914, PSR J1826-1256, and PSR J2129+1210A); for two of these the spin-down limit was potentially within reach using our data.

The ephemerides have been created using pulse time-ofarrival observations that mainly overlapped with all, or some fraction of, the $\mathrm{O} 1$ and $\mathrm{O} 2$ observing periods (see Section 2.1), so the timing solutions should provide coherent phase models over and between the two runs. Of the 222, we have 168 for which the electromagnetic timings fully overlapped with the full $\mathrm{O} 1$ and $\mathrm{O} 2$ runs. There are 12 pulsars for which there is no overlap between electromagnetic observations and the $\mathrm{O} 2$ run. These include two pulsars, J1412+7922 (known as Calvera) and J1849-0001, for which we only have X-ray timing observations from after O2 (Bogdanov et al. 2019). ${ }^{198}$ For these we have made the reasonable assumption that timing models are coherent for our analysis and that no timing irregularities, such as glitches, are present.

In all previous searches a total of 271 pulsars had been searched for, with 167 of these being timed for this search. For the other sources ephemerides were not available to us for our current analysis. In particular, we do not have up-to-date ephemerides for many of the pulsars in the globular clusters 47 Tucanae and Terzan 5, or the interesting young X-ray pulsar J0537-6910.

\subsubsection{Glitches}

During the course of the $\mathrm{O} 2$ period, five pulsars exhibited timing glitches. The Vela pulsar (J0835-4510) glitched on 2016 December 12 at 11:36 UTC (Palfreyman 2016; Palfreyman et al. 2018), and the Crab pulsar (J0534+2200) showed a small glitch on 2017 March 27 at around 22:04 UTC (Espinoza et al. 2011). ${ }^{199}$ PSR J1028-5819 glitched some time around 2017 May 29, with a best-fit glitch time of 01:36 UTC. PSR J1718-3825 experienced a small glitch around 2017 July 2. PSR J0205+6449 experienced four glitches over the period between the start of $\mathrm{O} 1$ and the end of $\mathrm{O} 2$, with glitch epochs of 2015 November 19, 2016 July 1, 2016 October 19, and 2017 May 27. Two of these glitches occurred in the period between $\mathrm{O} 1$ and $\mathrm{O} 2$, and as such any effect of the glitches on discrepancies between the electromagnetic and gravitationalwave phase would not be independently distinguishable, meaning that effectively only three glitches need to be accounted for.

\footnotetext{
198 Subsequent to the search performed here, Bogdanov et al. (2019) revised their initial timing model of J1849-0001 so that it now overlaps partially with O2. The revised model is consistent with the initial model used here, and thus the results presented here remain valid.

199 http://www.jb.man.ac.uk/pulsar/glitches.html
} 


\subsubsection{Timing Noise}

Timing noise is low-frequency noise observed in the residuals of pulsar pulse arrival times after subtracting a loworder Taylor expansion fit (see, e.g., Hobbs et al. 2006a). As shown in Cordes \& Helfand (1980), Arzoumanian et al. (1994) timing noise is strongly correlated with pulsar period derivative, so "young," or canonical, pulsars generally have far higher levels than MSPs. If not accounted for in the timing model, the Crab pulsar's phase, for example, could deviate by on the order of a cycle over the course of our observations, leading to decoherence of the signal (see Jones 2004; Pitkin \& Woan 2007; Ashton et al. 2015). In our gravitationalwave searches we used phase models that incorporate the effects of timing noise when necessary. In some cases this is achieved by using a phase model that includes high-order coefficients in the Taylor expansion (including up to the twelfth frequency derivative in the case of the Crab pulsar) when fitting the electromagnetic pulse arrival times. In others, where expansions in the phase do not perform well, we have used the method of fitting multiple sinusoidal harmonics to the timing noise in the arrival times, as described in Hobbs et al. (2004) and implemented in the FITWAVES algorithm in TEMPO2 (Hobbs et al. 2006b).

\subsubsection{Distances and Period Derivatives}

When calculating results of the searches in terms of the $Q_{22}$ mass quadrupole, fiducial ellipticity, or spin-down limits (see Appendix A), we require the distances to the pulsars. For the majority of pulsars we use "best-estimate" distances given in the ATNF Pulsar Catalog (Manchester et al. 2005). ${ }^{200}$ In the majority of cases these are distances based on the observed dispersion measure and calculated using the Galactic electron density distribution model of Yao et al. (2017), although others are based on parallax measurements, or inferred from associations with other objects or flux measurements. The distances used for each pulsar, as well as the reference for the value used, are given in Tables 1 and 2 .

The spin-down limits that we compare our results to (see Appendix A) require a value for the first period derivative $\dot{P}$, or equivalently frequency derivative $\dot{f}$, of the pulsar. The observed spin-down does not necessarily reflect the intrinsic spin-down of the pulsar, as it can be contaminated by the relative motion of the pulsar with respect to the observer. This is particularly prevalent for MSPs, which have intrinsically small spin-downs that can be strongly affected, particularly if they are in the core of a globular cluster where significant intracluster accelerations can occur, or if they have a large transverse velocity with respect to the solar system and/or are close (the "Shklovskii effect"; Shklovskii 1970.) The spindown can also be contaminated by the differential motion of the solar system and pulsar due to their orbits around the Galaxy. For the non-globular-cluster pulsars, if their proper motions and distances are well enough measured, then these effects can be corrected for to give the intrinsic period derivative (see, e.g., Damour \& Taylor 1991). For pulsars where the intrinsic period derivative is given in the literature we have used those values (see Tables 1 and 2 for the values and associated references). For further non-globular-cluster pulsars for which a transverse velocity and distance are given in

\footnotetext{
${ }^{200}$ Version 1.59 of the catalog available at http://www.atnf.csiro.au/people/ pulsar/psrcat/.
}

the ATNF Pulsar Catalog, we correct the observed period derivative using the method in Damour \& Taylor (1991). In some cases the corrections lead to negative period derivative values, indicating that the true values are actually too small to be confidently constrained. For these cases Table 2 does not give a period derivative value or associated spin-down limit.

As was previously done in Abbott et al. (2017a), for two globular cluster pulsars, J1823-3021A and J1824-2452A, we assume that the observed spin-down is not significantly contaminated by cluster effects following the discussions in Freire et al. (2011) and Johnson et al. (2013), respectively, so these values are used without any correction. For the other globular cluster pulsars, we again take the approach of Aasi et al. (2014) and Abbott et al. (2017a) and create proxy period derivative values by assuming that the stars have characteristic ages of $10^{9} \mathrm{yr}$ and braking indices of $n=5$ (i.e., they are braked purely by gravitational radiation from the $l=m=2$ mode). ${ }^{201}$

\subsubsection{Orientation Constraints}

In $\mathrm{Ng}$ \& Romani (2004, 2008) models are fitted to a selection of X-ray observations of pulsar wind nebulae, which are used to provide the orientations of the nebulae. In previous gravitational-wave searches (Abbott et al. 2008, 2010, 2017a; Aasi et al. 2014) the assumption has been made that the orientation of the wind nebula is consistent with the orientation of its pulsar. In this work we will also follow this assumption and use the fits in $\mathrm{Ng} \&$ Romani (2008) as prior constraints on orientation (inclination angle $\iota$ and polarization angle $\psi$ ) for PSR J0205+6449, PSR J0534+2200, PSR J0835-4510, PSR $\mathrm{J} 1952+3252$, and PSR J2229+6114. This is discussed in more detail in Appendix B. We refer to results based on these constraints as using restricted priors.

Constraints on the position angle, and therefore gravitational-wave polarization angle, of pulsars are also possible through observations of their electromagnetic polarization (Johnston et al. 2005). None of the pulsars in Johnston et al. (2005) are in our target list, but such constraints may be useful in the future. Constraints on the polarization angle alone are not as useful as those that also provide the inclination of the source (as described above for the pulsar wind nebula observations), which is directly correlated with the gravitational-wave amplitude. However, there are some pulsars for which double pulses are observed (Kramer \& Johnston 2008; Keith et al. 2010), suggesting that the rotation axis and magnetic axis are orthogonal, and therefore implying an inclination angle of $\iota \approx \pm 90^{\circ}$. In terms of upper limits on the gravitationalwave amplitude, the implication of $\iota \approx 90^{\circ}$ would generally be to lead to a larger limit on $h_{0}$ than for an inclination aligned with the line of sight, due to the relatively weaker observed strain for a linearly polarized signal compared to a circularly polarized signal of the same $h_{0}$. Of the pulsars observed in Keith et al. (2010), one (PSR J1828-1101) is in our search, although we have not used the implied constraints in this analysis. In the future these constraints will be considered if appropriate.

\footnotetext{
201 The braking index $n$ defines the power-law relation between the pulsar's frequency and frequency derivative via $\dot{f}=-k f^{n}$, where $k$ is a constant. Purely magnetic dipole braking gives a value of $n=3$, and purely quadrupole gravitational-wave braking gives $n=5$. The characteristic age is defined as $\tau=(n-1)^{-1}(f / \dot{f})$.
} 
Table 1

Limits on Gravitational-wave Amplitude, and Other Derived Quantities, for 34 High-value Pulsars from the Three Analysis Methods

\begin{tabular}{|c|c|c|c|c|c|c|c|c|c|c|c|c|c|}
\hline $\begin{array}{l}\text { Pulsar Name } \\
\text { (J2000) }\end{array}$ & $\begin{array}{l}f_{\mathrm{rot}} \\
(\mathrm{Hz})\end{array}$ & $\underset{\left(\mathrm{s} \mathrm{s}^{-1}\right)}{\dot{P}_{\text {of }}}$ & $\begin{array}{l}\text { Distance } \\
(\mathrm{kpc})\end{array}$ & $h_{0}^{\text {sd }}$ & $\begin{array}{l}\text { Analysis } \\
\text { Method }\end{array}$ & $C_{21}^{95 \%}$ & $C_{22}^{95 \%}$ & $h_{0}^{95 \%}$ & $\begin{array}{c}Q_{22}^{95 \%} \\
\left(\mathrm{~kg} \mathrm{~m}^{2}\right)\end{array}$ & $\varepsilon^{95 \%}$ & $h_{0}^{95 \%} / h_{0}^{\text {sd }}$ & $\begin{array}{c}\text { Statistic }^{\mathrm{a}} \\
l=2, m=1,2\end{array}$ & $\begin{array}{c}\begin{array}{c}\text { Statistic } \\
l=2, m=2\end{array} \\
\text { b }\end{array}$ \\
\hline $\mathrm{J} 0030+0451$ & 205.5 & $1.1 \times 10^{-20} \mathrm{~g}$ & 0.33 (a) & $3.7 \times 10^{-27}$ & $\begin{array}{c}\text { Bayesian } \\
\mathcal{F} \text {-statistic }\end{array}$ & $\begin{array}{c}1.7 \times 10^{-26} \\
\ldots\end{array}$ & $\begin{array}{c}5.9 \times 10^{-27} \\
\ldots\end{array}$ & $\begin{array}{c}1.3 \times 10^{-26} \\
\ldots\end{array}$ & $\begin{array}{c}1.8 \times 10^{30} \\
\ldots\end{array}$ & $\begin{array}{c}2.3 \times 10^{-8} \\
\ldots\end{array}$ & $\begin{array}{l}3.4 \\
\ldots\end{array}$ & $\begin{array}{c}-3.8 \\
\ldots\end{array}$ & $\begin{array}{c}-2.1 \\
\ldots\end{array}$ \\
\hline & & & & & $5 n$-vector & $1.3 \times 10^{-26}$ & $\ldots$ & $1.7 \times 10^{-26}$ & $2.3 \times 10^{30}$ & $3.0 \times 10^{-8}$ & 4.5 & 0.72 & 0.61 \\
\hline $\mathrm{J} 0117+5914^{\mathrm{c}}$ & 9.9 & $5.9 \times 10^{-15}$ & 1.7 (b) & $1.1 \times 10^{-25}$ & $\begin{array}{c}\text { Bayesian } \\
\mathcal{F} \text {-statistic } \\
5 n \text {-vector }\end{array}$ & $\begin{array}{l}\cdots \\
\cdots \\
\cdots\end{array}$ & $\begin{array}{l}\cdots \\
\ldots \\
\ldots\end{array}$ & $\begin{array}{c}3.8 \times 10^{-25} \\
\cdots \\
2.6 \times 10^{-25}\end{array}$ & $\begin{array}{c}1.3 \times 10^{35} \\
\cdots \\
8.6 \times 10^{34}\end{array}$ & $\begin{array}{c}1.7 \times 10^{-3} \\
\cdots \\
1.1 \times 10^{-3}\end{array}$ & $\begin{array}{l}3.5 \\
\ldots \\
2.4\end{array}$ & $\begin{array}{l}-2.4 \\
\ldots \\
\ldots\end{array}$ & $\begin{array}{c}-1.9 \\
\cdots \\
0.31\end{array}$ \\
\hline $\mathrm{J} 0205+6449^{\mathrm{c}}$ & 15.2 & $1.9 \times 10^{-13}$ & $2.00(\mathrm{c})$ & $6.9 \times 10^{-25}$ & $\begin{array}{c}\text { Bayesian } \\
\mathcal{F} \text {-statistic } \\
5 n \text {-vector }\end{array}$ & $\begin{array}{c}1.8(1.5) \times 10^{-24} \\
2.2 \times 10^{-24} \\
\ldots\end{array}$ & $\begin{array}{c}2.4(3.6) \times 10^{-26} \\
4.5 \times 10^{-26} \\
\ldots\end{array}$ & $\begin{array}{c}4.9(7.1) \times 10^{-26} \\
8.8 \times 10^{-26} \\
2.9(4.5) \times 10^{-26}\end{array}$ & $\begin{array}{c}0.8(1.1) \times 10^{33} \\
1.4 \times 10^{34} \\
4.6(7.1) \times 10^{33}\end{array}$ & $\begin{array}{c}1.0(1.5) \times 10^{-4} \\
1.8 \times 10^{-4} \\
5.9(9.2) \times 10^{-5}\end{array}$ & $\begin{array}{c}0.071(0.1) \\
0.13 \\
0.042(0.065)\end{array}$ & $\begin{array}{c}-4.8(-4.6) \\
0.71 \\
\ldots\end{array}$ & $\begin{array}{c}-2.7(-2.4) \\
0.26 \\
0.41\end{array}$ \\
\hline $\mathrm{J} 0534+2200^{\mathrm{c}}$ & 29.7 & $4.2 \times 10^{-13}$ & 2.00 & $1.4 \times 10^{-24}$ & $\begin{array}{c}\text { Bayesian } \\
\mathcal{F} \text {-statistic } \\
5 n \text {-vector }\end{array}$ & $\begin{array}{l}7.9(5.8) \times 10^{-26} \\
1.6(1.1) \times 10^{-25} \\
1.7(1.3) \times 10^{-25}\end{array}$ & $\begin{array}{c}9.1(7.3) \times 10^{-27} \\
1.1(1.1) \times 10^{-26} \\
\ldots\end{array}$ & $\begin{array}{l}1.9(1.5) \times 10^{-26} \\
2.2(1.3) \times 10^{-26} \\
2.9(2.9) \times 10^{-26}\end{array}$ & $\begin{array}{l}7.7(6.0) \times 10^{32} \\
9.1(5.4) \times 10^{32} \\
1.2(1.2) \times 10^{33}\end{array}$ & $\begin{array}{l}1.0(0.8) \times 10^{-5} \\
1.2(0.7) \times 10^{-5} \\
1.6(1.6) \times 10^{-5}\end{array}$ & $\begin{array}{c}0.013(0.01) \\
0.015(0.0091) \\
0.02(0.02)\end{array}$ & $\begin{array}{c}-5.1(-5.2) \\
0.32(0.18) \\
0.70\end{array}$ & $\begin{array}{c}-2.6(-2.7) \\
0.65(0.87) \\
0.45\end{array}$ \\
\hline $\mathrm{J} 0711-6830^{\mathrm{c}}$ & 182.1 & $1.4 \times 10^{-20}$ & 0.11 (b) & $1.2 \times 10^{-26}$ & $\begin{array}{c}\text { Bayesian } \\
\mathcal{F} \text {-statistic } \\
5 n \text {-vector }\end{array}$ & $\begin{array}{c}2.6 \times 10^{-26} \\
\cdots \\
1.2 \times 10^{-26}\end{array}$ & $\begin{array}{c}7.0 \times 10^{-27} \\
\quad \ldots \\
\ldots\end{array}$ & $\begin{array}{c}1.5 \times 10^{-26} \\
\cdots \\
1.5 \times 10^{-26}\end{array}$ & $\begin{array}{c}9.3 \times 10^{29} \\
\quad \cdots \\
9.1 \times 10^{29}\end{array}$ & $\begin{array}{c}1.2 \times 10^{-8} \\
\cdots \\
1.2 \times 10^{-8}\end{array}$ & $\begin{array}{l}1.3 \\
\ldots \\
1.3\end{array}$ & $\begin{array}{c}-3.1 \\
\ldots \\
0.79\end{array}$ & $\begin{array}{c}-1.9 \\
\ldots \\
0.39\end{array}$ \\
\hline $\mathrm{J} 0835-4510^{\mathrm{c}}$ & 11.2 & $1.2 \times 10^{-13}$ & $0.29(\mathrm{j})$ & $3.3 \times 10^{-24}$ & $\begin{array}{c}\text { Bayesian } \\
\mathcal{F} \text {-statistic } \\
5 n \text {-vector }\end{array}$ & $\begin{array}{c}1.4(1.1) \times 10^{-23} \\
1.3(1.1) \times 10^{-23} \\
\ldots\end{array}$ & $\begin{array}{c}6.7(6.2) \times 10^{-26} \\
1.1(0.9) \times 10^{-25} \\
\ldots\end{array}$ & $\begin{array}{l}1.4(1.2) \times 10^{-25} \\
2.6(2.0) \times 10^{-25} \\
2.3(2.4) \times 10^{-25}\end{array}$ & $\begin{array}{l}5.9(5.2) \times 10^{33} \\
1.1(0.8) \times 10^{34} \\
9.7(9.9) \times 10^{33}\end{array}$ & $\begin{array}{l}7.6(6.7) \times 10^{-5} \\
1.4(1.1) \times 10^{-4} \\
1.3(1.3) \times 10^{-4}\end{array}$ & $\begin{array}{c}0.042(0.037) \\
0.078(0.06) \\
0.07(0.071)\end{array}$ & $\begin{array}{c}-4.2(-4.4) \\
0.75(0.75) \\
\ldots\end{array}$ & $\begin{array}{c}-2.5(-2.8) \\
0.75(0.75) \\
0.41\end{array}$ \\
\hline J0940-5428 & 11.4 & $3.3 \times 10^{-14}$ & 0.38 (b) & $1.3 \times 10^{-24}$ & $\begin{array}{c}\text { Bayesian } \\
\mathcal{F} \text {-statistic } \\
5 n \text {-vector }\end{array}$ & $\begin{array}{c}1.6 \times 10^{-23} \\
\ldots \\
\ldots\end{array}$ & $\begin{array}{c}7.7 \times 10^{-26} \\
\ldots \\
\ldots\end{array}$ & $\begin{array}{c}1.6 \times 10^{-25} \\
\cdots \\
1.7 \times 10^{-25}\end{array}$ & $\begin{array}{c}8.7 \times 10^{33} \\
\cdots \\
8.9 \times 10^{33}\end{array}$ & $\begin{array}{c}1.1 \times 10^{-4} \\
\ldots \\
1.2 \times 10^{-4}\end{array}$ & $\begin{array}{c}0.13 \\
\cdots \\
0.13\end{array}$ & $\begin{array}{l}-3.7 \\
\ldots \\
\ldots\end{array}$ & $\begin{array}{c}-2.3 \\
\ldots \\
0.70\end{array}$ \\
\hline J1028-5819 & 10.9 & $1.6 \times 10^{-14}$ & 1.42 (b) & $2.4 \times 10^{-25}$ & $\begin{array}{l}\text { Bayesian } \\
\mathcal{F} \text {-statistic } \\
5 n \text {-vector }\end{array}$ & $\begin{array}{c}2.7 \times 10^{-23} \\
\ldots \\
\ldots\end{array}$ & $\begin{array}{c}9.1 \times 10^{-26} \\
\ldots \\
\ldots\end{array}$ & $\begin{array}{c}2.3 \times 10^{-25} \\
\cdots \\
1.9 \times 10^{-25}\end{array}$ & $\begin{array}{c}5.1 \times 10^{34} \\
\cdots \\
4.1 \times 10^{34}\end{array}$ & $\begin{array}{c}6.6 \times 10^{-4} \\
\cdots \\
5.3 \times 10^{-4}\end{array}$ & $\begin{array}{c}0.98 \\
\ldots \\
0.8\end{array}$ & $\begin{array}{c}-3.5 \\
\ldots \\
\ldots\end{array}$ & $\begin{array}{c}-2.2 \\
\ldots \\
0.40\end{array}$ \\
\hline J1105-6107 & 15.8 & $1.6 \times 10^{-14}$ & 2.36 (b) & $1.7 \times 10^{-25}$ & $\begin{array}{c}\text { Bayesian } \\
\mathcal{F} \text {-statistic } \\
5 n \text {-vector }\end{array}$ & $\begin{array}{c}1.7 \times 10^{-24} \\
\ldots \\
\ldots\end{array}$ & $\begin{array}{c}2.0 \times 10^{-26} \\
\ldots \\
\ldots\end{array}$ & $\begin{array}{c}3.9 \times 10^{-26} \\
\cdots \\
2.7 \times 10^{-26}\end{array}$ & $\begin{array}{c}6.7 \times 10^{33} \\
\ldots \\
4.6 \times 10^{33}\end{array}$ & $\begin{array}{c}8.7 \times 10^{-5} \\
\cdots \\
6.0 \times 10^{-5}\end{array}$ & $\begin{array}{c}0.23 \\
\ldots \\
0.16\end{array}$ & $\begin{array}{c}-4.6 \\
\ldots \\
\ldots\end{array}$ & $\begin{array}{c}-2.8 \\
\ldots \\
0.93\end{array}$ \\
\hline $\mathrm{J} 1112-6103$ & 15.4 & $3.1 \times 10^{-14}$ & 4.50 (b) & $1.2 \times 10^{-25}$ & $\begin{array}{l}\text { Bayesian } \\
\mathcal{F} \text {-statistic }\end{array}$ & $\begin{array}{c}3.4 \times 10^{-24} \\
\ldots\end{array}$ & $\begin{array}{c}2.5 \times 10^{-26} \\
\cdots\end{array}$ & $\begin{array}{c}5.8 \times 10^{-26} \\
\ldots\end{array}$ & $\begin{array}{c}2.0 \times 10^{34} \\
\ldots\end{array}$ & $\begin{array}{c}2.6 \times 10^{-4} \\
\ldots\end{array}$ & $\begin{array}{c}0.47 \\
\ldots\end{array}$ & $\begin{array}{c}-4.2 \\
\ldots\end{array}$ & $\begin{array}{c}-3.4 \\
\ldots\end{array}$ \\
\hline $\mathrm{J} 1410-6132$ & 20.0 & $3.2 \times 10^{-14}$ & 13.51 (b) & $4.8 \times 10^{-26}$ & $\begin{array}{c}5 n \text {-vector } \\
\text { Bayesian } \\
\mathcal{F} \text {-statistic } \\
5 n \text {-vector }\end{array}$ & $\begin{array}{c}\cdots \\
4.9 \times 10^{-25} \\
\cdots \\
5.4 \times 10^{-25}\end{array}$ & $\begin{array}{c}\cdots \\
9.4 \times 10^{-27} \\
\ldots \\
\ldots\end{array}$ & $\begin{array}{c}3.6 \times 10^{-26} \\
2.1 \times 10^{-26} \\
\quad \cdots \\
2.6 \times 10^{-26}\end{array}$ & $\begin{array}{c}1.2 \times 10^{34} \\
1.3 \times 10^{34} \\
\quad \ldots \\
1.6 \times 10^{34}\end{array}$ & $\begin{array}{c}1.6 \times 10^{-4} \\
1.7 \times 10^{-4} \\
\quad \cdots \\
2.1 \times 10^{-4}\end{array}$ & $\begin{array}{c}0.29 \\
0.44 \\
\ldots \\
0.55\end{array}$ & $\begin{array}{c}\ldots \\
-5.7 \\
\ldots \\
\ldots\end{array}$ & $\begin{array}{c}0.76 \\
-3.0 \\
\ldots \\
0.88\end{array}$ \\
\hline $\mathrm{J} 1412+7922$ & 16.9 & $3.3 \times 10^{-15}$ & $2.00(\mathrm{o})$ & $9.5 \times 10^{-26}$ & $\begin{array}{c}\text { Bayesian } \\
\mathcal{F} \text {-statistic } \\
5 n \text {-vector }\end{array}$ & $\begin{array}{c}1.8 \times 10^{-24} \\
2.3 \times 10^{-24} \\
\ldots\end{array}$ & $\begin{array}{c}3.4 \times 10^{-26} \\
2.2 \times 10^{-26} \\
\ldots\end{array}$ & $\begin{array}{l}7.5 \times 10^{-26} \\
6.2 \times 10^{-26} \\
3.6 \times 10^{-26}\end{array}$ & $\begin{array}{l}9.6 \times 10^{33} \\
7.9 \times 10^{33} \\
4.6 \times 10^{33}\end{array}$ & $\begin{array}{l}1.2 \times 10^{-4} \\
1.0 \times 10^{-4} \\
6.0 \times 10^{-5}\end{array}$ & $\begin{array}{l}0.78 \\
0.65 \\
0.38\end{array}$ & $\begin{array}{c}-4.9 \\
0.24 \\
\ldots\end{array}$ & $\begin{array}{l}-2.1 \\
0.39 \\
0.80\end{array}$ \\
\hline $\mathrm{J} 1420-6048$ & 14.8 & $8.3 \times 10^{-14}$ & 5.63 (b) & $1.6 \times 10^{-25}$ & $\begin{array}{c}\text { Bayesian } \\
\mathcal{F} \text {-statistic } \\
5 n \text {-vector }\end{array}$ & $\begin{array}{c}2.1 \times 10^{-24} \\
\ldots \\
\ldots\end{array}$ & $\begin{array}{c}1.9 \times 10^{-26} \\
\ldots \\
\ldots\end{array}$ & $\begin{array}{c}4.1 \times 10^{-26} \\
\cdots \\
7.6 \times 10^{-26}\end{array}$ & $\begin{array}{c}1.9 \times 10^{34} \\
\cdots \\
3.6 \times 10^{34}\end{array}$ & $\begin{array}{c}2.5 \times 10^{-4} \\
\cdots \\
4.7 \times 10^{-4}\end{array}$ & $\begin{array}{c}0.26 \\
\ldots \\
0.48\end{array}$ & $\begin{array}{c}-6.2 \\
\ldots \\
\ldots\end{array}$ & $\begin{array}{c}-2.8 \\
\ldots \\
0.52\end{array}$ \\
\hline $\mathrm{J} 1509-5850$ & 11.2 & $9.2 \times 10^{-15}$ & 3.37 (b) & $7.7 \times 10^{-26}$ & $\begin{array}{c}\text { Bayesian } \\
\mathcal{F} \text {-statistic } \\
5 n \text {-vector }\end{array}$ & $\begin{array}{c}1.7 \times 10^{-23} \\
\ldots \\
\ldots\end{array}$ & $\begin{array}{c}1.5 \times 10^{-25} \\
\ldots \\
\ldots\end{array}$ & $\begin{array}{c}5.4 \times 10^{-25} \\
\cdots \\
2.1 \times 10^{-25}\end{array}$ & $\begin{array}{c}2.6 \times 10^{35} \\
\cdots \\
1.0 \times 10^{35}\end{array}$ & $\begin{array}{c}3.4 \times 10^{-3} \\
\cdots \\
1.3 \times 10^{-3}\end{array}$ & $\begin{array}{l}7.1 \\
\ldots \\
2.7\end{array}$ & $\begin{array}{l}-3.5 \\
\ldots \\
\ldots\end{array}$ & $\begin{array}{c}-2.0 \\
\ldots \\
0.72\end{array}$ \\
\hline $\mathrm{J} 1531-5610$ & 11.9 & $1.4 \times 10^{-14}$ & 2.84 (b) & $1.1 \times 10^{-25}$ & $\begin{array}{c}\text { Bayesian } \\
\mathcal{F} \text {-statistic } \\
5 n \text {-vector }\end{array}$ & $\begin{array}{c}7.9 \times 10^{-24} \\
\ldots \\
\ldots\end{array}$ & $\begin{array}{c}5.5 \times 10^{-26} \\
\ldots \\
\ldots\end{array}$ & $\begin{array}{c}1.2 \times 10^{-25} \\
\cdots \\
1.4 \times 10^{-25}\end{array}$ & $\begin{array}{c}4.4 \times 10^{34} \\
\cdots \\
5.3 \times 10^{34}\end{array}$ & $\begin{array}{c}5.6 \times 10^{-4} \\
\cdots \\
6.8 \times 10^{-4}\end{array}$ & $\begin{array}{l}1 \\
\ldots \\
1.2\end{array}$ & $\begin{array}{l}-4.2 \\
\ldots \\
\ldots\end{array}$ & $\begin{array}{c}-2.4 \\
\ldots \\
0.31\end{array}$ \\
\hline
\end{tabular}


Table 1

(Continued)

\begin{tabular}{|c|c|c|c|c|c|c|c|c|c|c|c|c|c|}
\hline $\begin{array}{l}\text { Pulsar Name } \\
\text { (J2000) }\end{array}$ & $\begin{array}{l}f_{\mathrm{rot}} \\
(\mathrm{Hz})\end{array}$ & $\underset{\left(\mathrm{s} \mathrm{s}^{-1}\right)}{\dot{P}_{\text {of }}}$ & $\begin{array}{l}\text { Distance } \\
(\mathrm{kpc})\end{array}$ & $h_{0}^{\text {sd }}$ & $\begin{array}{l}\text { Analysis } \\
\text { Method }\end{array}$ & $C_{21}^{95 \%}$ & $C_{22}^{95 \%}$ & $h_{0}^{95 \%}$ & $\begin{array}{l}Q_{22}^{95 \%} \\
\left(\mathrm{~kg} \mathrm{~m}^{2}\right)\end{array}$ & $\varepsilon^{95 \%}$ & $h_{0}^{95 \%} / h_{0}^{\mathrm{sd}}$ & $\begin{array}{c}\text { Statistic }^{\mathrm{a}} \\
l=2, m=1,2\end{array}$ & $\underset{l=2, m=2}{\text { Statistic }^{\mathrm{b}}}$ \\
\hline \multirow[t]{2}{*}{$\mathrm{J} 1718-3825$} & 13.4 & $1.3 \times 10^{-14}$ & 3.49 (b) & $9.7 \times 10^{-26}$ & $\begin{array}{c}\text { Bayesian } \\
\mathcal{F} \text {-statistic }\end{array}$ & $\begin{array}{c}3.2 \times 10^{-24} \\
\ldots\end{array}$ & $\begin{array}{c}4.2 \times 10^{-26} \\
\ldots\end{array}$ & $\begin{array}{c}8.7 \times 10^{-26} \\
\ldots\end{array}$ & $\begin{array}{c}3.1 \times 10^{34} \\
\ldots\end{array}$ & $\begin{array}{c}4.0 \times 10^{-4} \\
\ldots\end{array}$ & $\begin{array}{l}0.9 \\
\ldots\end{array}$ & $\begin{array}{c}-5.6 \\
\ldots\end{array}$ & $\begin{array}{l}-2.4 \\
\ldots\end{array}$ \\
\hline & & & & & $5 n$-vector & $\ldots$ & $\ldots$ & $6.5 \times 10^{-26}$ & $2.3 \times 10^{34}$ & $3.0 \times 10^{-4}$ & 0.67 & $\ldots$ & 0.67 \\
\hline \multirow[t]{3}{*}{ J1809-1917 } & 12.1 & $2.6 \times 10^{-14}$ & 3.27 (b) & $1.4 \times 10^{-25}$ & Bayesian & $6.6 \times 10^{-24}$ & $4.9 \times 10^{-26}$ & $9.8 \times 10^{-26}$ & $4.0 \times 10^{34}$ & $5.2 \times 10^{-4}$ & 0.72 & -4.4 & -2.5 \\
\hline & & & & & $\mathcal{F}$-statistic & $6.2 \times 10^{-24}$ & $6.2 \times 10^{-26}$ & $7.3 \times 10^{-26}$ & $3.0 \times 10^{34}$ & $3.9 \times 10^{-4}$ & 0.53 & 0.76 & 0.76 \\
\hline & & & & & $5 n$-vector & $\ldots$ & $\ldots$ & $1.1 \times 10^{-25}$ & $4.3 \times 10^{34}$ & $5.6 \times 10^{-4}$ & 0.77 & $\ldots$ & 0.19 \\
\hline \multirow[t]{3}{*}{ J1813-1246 } & 20.8 & $1.8 \times 10^{-14}$ & $2.50(\mathrm{z})$ & $1.9 \times 10^{-25}$ & Bayesian & $3.9 \times 10^{-25}$ & $2.2 \times 10^{-26}$ & $4.7 \times 10^{-26}$ & $5.0 \times 10^{33}$ & $6.4 \times 10^{-5}$ & 0.24 & -4.2 & -2.2 \\
\hline & & & & & $\mathcal{F}$-statistic & $3.8 \times 10^{-25}$ & $1.0 \times 10^{-26}$ & $3.3 \times 10^{-26}$ & $3.5 \times 10^{33}$ & $4.5 \times 10^{-5}$ & 0.17 & 0.08 & 0.73 \\
\hline & & & & & $5 n$-vector & $1.0 \times 10^{-24}$ & $\ldots$ & $4.5 \times 10^{-26}$ & $4.7 \times 10^{33}$ & $6.1 \times 10^{-5}$ & 0.23 & $\ldots$ & 0.22 \\
\hline \multirow[t]{3}{*}{ J1826-1256 } & 9.1 & $1.2 \times 10^{-13}$ & $1.39(\mathrm{cc})$ & $6.1 \times 10^{-25}$ & Bayesian & $\ldots$ & $\ldots$ & $6.2 \times 10^{-25}$ & $1.9 \times 10^{35}$ & $2.5 \times 10^{-3}$ & 1 & -2.0 & -2.1 \\
\hline & & & & & $\mathcal{F}$-statistic & $\ldots$ & $\ldots$ & $\ldots$ & $\ldots$ & $\ldots$ & $\ldots$ & $\ldots$ & $\ldots$ \\
\hline & & & & & $5 n$-vector & $\cdots$ & $\ldots$ & $4.7 \times 10^{-25}$ & $1.5 \times 10^{35}$ & $1.9 \times 10^{-3}$ & 0.77 & $\ldots$ & $\cdots$ \\
\hline \multirow[t]{3}{*}{$\mathrm{J} 1828-1101$} & 13.9 & $1.5 \times 10^{-14}$ & 4.77 (b) & $7.7 \times 10^{-26}$ & Bayesian & $7.5 \times 10^{-24}$ & $4.6 \times 10^{-26}$ & $7.2 \times 10^{-26}$ & $3.3 \times 10^{34}$ & $4.2 \times 10^{-4}$ & 0.94 & -4.6 & -2.5 \\
\hline & & & & & $\mathcal{F}$-statistic & $\ldots$ & $\ldots$ & $\ldots$ & $\ldots$ & $\ldots$ & $\ldots$ & $\ldots$ & $\ldots$ \\
\hline & & & & & $5 n$-vector & $\ldots$ & $\ldots$ & $5.5 \times 10^{-26}$ & $2.5 \times 10^{34}$ & $3.2 \times 10^{-4}$ & 0.71 & $\ldots$ & 0.13 \\
\hline \multirow[t]{3}{*}{ J1831-0952 } & 14.9 & $8.3 \times 10^{-15}$ & 3.68 (b) & $7.7 \times 10^{-26}$ & Bayesian & $3.2 \times 10^{-24}$ & $3.1 \times 10^{-26}$ & $6.9 \times 10^{-26}$ & $2.1 \times 10^{34}$ & $2.7 \times 10^{-4}$ & 0.9 & -5.0 & -2.4 \\
\hline & & & & & $\mathcal{F}$-statistic & $\ldots$ & $\ldots$ & $\ldots$ & $\ldots$ & $\ldots$ & $\ldots$ & $\ldots$ & $\ldots$ \\
\hline & & & & & $5 n$-vector & $\ldots$ & $\ldots$ & $4.3 \times 10^{-26}$ & $1.3 \times 10^{34}$ & $1.7 \times 10^{-4}$ & 0.56 & $\ldots$ & 0.75 \\
\hline \multirow[t]{3}{*}{$\mathrm{J} 1833-0827^{\mathrm{c}}$} & 11.7 & $9.2 \times 10^{-15}$ & $4.50(\mathrm{~m})$ & $5.9 \times 10^{-26}$ & Bayesian & $1.9 \times 10^{-23}$ & $8.8 \times 10^{-26}$ & $3.3 \times 10^{-25}$ & $2.0 \times 10^{35}$ & $2.6 \times 10^{-3}$ & 5.6 & -3.3 & -1.9 \\
\hline & & & & & $\mathcal{F}_{\text {-statistic }}$ & $\ldots$ & $\ldots$ & $\ldots$ & $\ldots$ & $\ldots$ & $\ldots$ & $\ldots$ & $\ldots$ \\
\hline & & & & & $5 n$-vector & $\ldots$ & $\ldots$ & $1.4 \times 10^{-25}$ & $8.3 \times 10^{34}$ & $1.1 \times 10^{-3}$ & 2.3 & $\ldots$ & 0.94 \\
\hline \multirow[t]{3}{*}{ J1837-0604 } & 10.4 & $4.5 \times 10^{-14}$ & 4.77 (b) & $1.2 \times 10^{-25}$ & Bayesian & $4.0 \times 10^{-23}$ & $1.1 \times 10^{-25}$ & $2.4 \times 10^{-25}$ & $1.9 \times 10^{35}$ & $2.5 \times 10^{-3}$ & 2 & -3.7 & -2.3 \\
\hline & & & & & $\mathcal{F}$-statistic & $\ldots$ & $\ldots$ & $\ldots$ & $\ldots$ & & $\ldots$ & $\ldots$ & $\ldots$ \\
\hline & & & & & $5 n$-vector & $\ldots$ & $\ldots$ & $1.6 \times 10^{-25}$ & $1.3 \times 10^{35}$ & $1.6 \times 10^{-3}$ & 1.4 & $\cdots$ & 0.38 \\
\hline \multirow[t]{3}{*}{$\mathrm{J} 1849-0001$} & 26.0 & $1.4 \times 10^{-14}$ & $7.00(\mathrm{dd})$ & $7.0 \times 10^{-26}$ & Bayesian & $7.1 \times 10^{-25}$ & $7.9 \times 10^{-27}$ & $1.9 \times 10^{-26}$ & $3.7 \times 10^{33}$ & $4.7 \times 10^{-5}$ & 0.28 & -3.4 & -2.6 \\
\hline & & & & & $\mathcal{F}$-statistic & $6.8 \times 10^{-25}$ & $9.1 \times 10^{-27}$ & $2.8 \times 10^{-26}$ & $5.3 \times 10^{33}$ & $6.9 \times 10^{-5}$ & 0.4 & 0.04 & 0.75 \\
\hline & & & & & $5 n$-vector & $6.8 \times 10^{-26}$ & $\ldots$ & $2.0 \times 10^{-26}$ & $3.8 \times 10^{33}$ & $4.9 \times 10^{-5}$ & 0.29 & 0.23 & 0.49 \\
\hline \multirow[t]{3}{*}{$\mathrm{J} 1856+0245$} & 12.4 & $6.2 \times 10^{-14}$ & 6.32 (b) & $1.1 \times 10^{-25}$ & Bayesian & $7.2 \times 10^{-24}$ & $7.3 \times 10^{-26}$ & $1.5 \times 10^{-25}$ & $1.1 \times 10^{35}$ & $1.4 \times 10^{-3}$ & 1.3 & -3.8 & -2.1 \\
\hline & & & & & $\mathcal{F}_{\text {-statistic }}$ & $\ldots$ & $\ldots$ & $\ldots$ & $\ldots$ & $\ldots$ & $\ldots$ & $\ldots$ & $\ldots$ \\
\hline & & & & & $5 n$-vector & $\ldots$ & $\ldots$ & $1.6 \times 10^{-25}$ & $1.2 \times 10^{35}$ & $1.6 \times 10^{-3}$ & 1.5 & $\ldots$ & 0.36 \\
\hline \multirow[t]{2}{*}{$\mathrm{J} 1913+1011$} & 27.8 & $3.4 \times 10^{-15}$ & 4.61 (b) & $5.4 \times 10^{-26}$ & Bayesian & $1.6 \times 10^{-25}$ & $1.8 \times 10^{-26}$ & $3.7 \times 10^{-26}$ & $4.0 \times 10^{33}$ & $5.2 \times 10^{-5}$ & 0.7 & -4.1 & -2.2 \\
\hline & & & & & $\begin{array}{c}\mathcal{F} \text {-statistic } \\
5 n \text {-vector }\end{array}$ & $\begin{array}{c}\cdots \\
1.7 \times 10^{-25}\end{array}$ & $\begin{array}{l}\ldots \\
\ldots\end{array}$ & $\begin{array}{c}\cdots \\
2.1 \times 10^{-26}\end{array}$ & $\begin{array}{c}\cdots \\
2.3 \times 10^{33}\end{array}$ & $3.0 \times 10^{-5}$ & $\begin{array}{c}\cdots \\
0.39\end{array}$ & 0.56 & $\begin{array}{c}\ldots \\
0.90\end{array}$ \\
\hline \multirow{3}{*}{$\mathrm{J} 1925+1720$} & 13.2 & $1.0 \times 10^{-14}$ & $5.06(\mathrm{~b})$ & $5.9 \times 10^{-26}$ & Bayesian & $3.3 \times 10^{-24}$ & $5.5 \times 10^{-26}$ & $1.1 \times 10^{-25}$ & $5.8 \times 10^{34}$ & & & & \\
\hline & 13.2 & $1.0 \times 10$ & 5.00 (D) & $5.9 \times 10$ & $\begin{array}{l}\mathcal{F} \text {-statistic } \\
\mathcal{F}\end{array}$ & $\begin{array}{c}3.3 \times 10 \\
\ldots\end{array}$ & $\begin{array}{c}5.5 \times 10 \\
\ldots\end{array}$ & $\begin{array}{c}1.1 \times 10 \\
\ldots\end{array}$ & $\begin{array}{c}5.8 \times 10 \\
\ldots\end{array}$ & $\begin{array}{c}7.5 \times 10 \\
\ldots\end{array}$ & $\begin{array}{l}1.9 \\
\ldots\end{array}$ & $\begin{array}{c}-5.6 \\
\ldots\end{array}$ & $\begin{array}{c}-2.4 \\
\ldots\end{array}$ \\
\hline & & & & & $5 n$-vector & $\ldots$ & $\ldots$ & $1.1 \times 10^{-25}$ & $5.8 \times 10^{34}$ & $7.5 \times 10^{-4}$ & 1.9 & $\ldots$ & 0.44 \\
\hline \multirow[t]{3}{*}{$\mathrm{J} 1928+1746$} & 14.5 & $1.3 \times 10^{-14}$ & 4.34 (b) & $8.1 \times 10^{-26}$ & Bayesian & $2.4 \times 10^{-24}$ & $5.5 \times 10^{-26}$ & $1.2 \times 10^{-25}$ & $4.3 \times 10^{34}$ & $5.6 \times 10^{-4}$ & 1.4 & -5.2 & -2.6 \\
\hline & & & & & $\mathcal{F}$-statistic & $2.2 \times 10^{-24}$ & $3.9 \times 10^{-26}$ & $1.3 \times 10^{-25}$ & $4.9 \times 10^{34}$ & $6.3 \times 10^{-4}$ & 1.6 & 0.61 & 0.61 \\
\hline & & & & & $5 n$-vector & $\ldots$ & $\ldots$ & $8.6 \times 10^{-26}$ & $3.2 \times 10^{34}$ & $4.2 \times 10^{-4}$ & 1.1 & $\ldots$ & 0.59 \\
\hline \multirow[t]{3}{*}{$\mathrm{J} 1935+2025$} & 12.5 & $6.1 \times 10^{-14}$ & 4.60 (b) & $1.5 \times 10^{-25}$ & Bayesian & $7.3 \times 10^{-24}$ & $5.2 \times 10^{-26}$ & $1.1 \times 10^{-25}$ & $6.2 \times 10^{34}$ & $8.0 \times 10^{-4}$ & 0.75 & -4.4 & -2.4 \\
\hline & & & & & $\mathcal{F}$-statistic & $5.0 \times 10^{-24}$ & $5.5 \times 10^{-26}$ & $1.3 \times 10^{-25}$ & $7.0 \times 10^{34}$ & $9.1 \times 10^{-4}$ & 0.85 & 0.71 & 0.71 \\
\hline & & & & & $5 n$-vector & $\ldots$ & $\ldots$ & $1.4 \times 10^{-25}$ & $7.6 \times 10^{34}$ & $9.8 \times 10^{-4}$ & 0.92 & $\ldots$ & 0.37 \\
\hline \multirow[t]{2}{*}{$\mathrm{J} 1952+3252^{\mathrm{C}}$} & 25.3 & $5.8 \times 10^{-15}$ & $3.00(\mathrm{~m})$ & $1.0 \times 10^{-25}$ & $\begin{array}{l}\text { Bayesian } \\
\mathcal{F} \text {-statistic }\end{array}$ & $\begin{array}{c}2.8(2.9) \times 10^{-25} \\
\ldots\end{array}$ & $\begin{array}{c}8.7(9.0) \times 10^{-27} \\
\ldots\end{array}$ & $\begin{array}{c}1.9(1.8) \times 10^{-26} \\
\ldots\end{array}$ & $\begin{array}{c}.7(1.5) \times 10^{33} \\
\ldots\end{array}$ & $\begin{array}{c}2.1(2.0) \times 10^{-5} \\
\ldots\end{array}$ & $\begin{array}{c}0.19(0.17) \\
\ldots\end{array}$ & $\begin{array}{c}-3.4(-3.5) \\
\ldots\end{array}$ & $\begin{array}{c}-2.7(-2.6) \\
\quad \ldots\end{array}$ \\
\hline & & & & & $5 n$-vector & $2.0(2.0) \times 10^{-25}$ & $\ldots$ & $2.4(2.5) \times 10^{-26}$ & $2.1(2.1) \times 10^{33}$ & $2.7(2.7) \times 10^{-5}$ & $0.24(0.24)$ & 0.06 & 0.70 \\
\hline
\end{tabular}




\begin{tabular}{|c|c|c|c|c|c|c|c|c|c|c|c|c|c|}
\hline $\begin{array}{l}\text { Pulsar Name } \\
\text { (J2000) }\end{array}$ & $\begin{array}{c}f_{\text {rot }} \\
(\mathrm{Hz})\end{array}$ & $\left(\mathrm{Ps} \mathrm{s}^{\dot{\mathrm{rot}}_{1}}\right)$ & $\begin{array}{l}\text { Distance } \\
(\mathrm{kpc})\end{array}$ & $h_{0}^{\text {sd }}$ & $\begin{array}{l}\text { Analysis } \\
\text { Method }\end{array}$ & $C_{21}^{95 \%}$ & $C_{22}^{95 \%}$ & $h_{0}^{95 \%}$ & $\begin{array}{l}Q_{22}^{95 \%} \\
\left(\mathrm{~kg} \mathrm{~m}^{2}\right)\end{array}$ & $\varepsilon^{95 \%}$ & $h_{0}^{95 \%} / h_{0}^{\text {sd }}$ & $\begin{array}{c}\text { Statistic } \\
l=2, m=1,2\end{array}$ & $\underset{l=2, m=2}{\text { Statistic }}$ \\
\hline $\mathrm{J} 2043+2740$ & 10.4 & $1.3 \times 10^{-15}$ & 1.48 (b) & $6.3 \times 10^{-26}$ & $\begin{array}{c}\text { Bayesian } \\
\mathcal{F} \text {-statistic } \\
5 n \text {-vector }\end{array}$ & $\begin{array}{c}2.6 \times 10^{-23} \\
2.1 \times 10^{-23} \\
\ldots\end{array}$ & $\begin{array}{c}7.3 \times 10^{-26} \\
6.4 \times 10^{-26} \\
\ldots\end{array}$ & $\begin{array}{l}1.6 \times 10^{-25} \\
2.8 \times 10^{-25} \\
1.9 \times 10^{-25}\end{array}$ & $\begin{array}{l}4.1 \times 10^{34} \\
7.0 \times 10^{34} \\
4.7 \times 10^{34}\end{array}$ & $\begin{array}{l}5.3 \times 10^{-4} \\
9.1 \times 10^{-4} \\
6.1 \times 10^{-4}\end{array}$ & $\begin{array}{c}2.6 \\
4.5 \\
3\end{array}$ & $\begin{array}{c}-4.2 \\
0.79 \\
\ldots\end{array}$ & $\begin{array}{l}-2.5 \\
0.79 \\
0.17\end{array}$ \\
\hline J2124-3358 & 202.8 & $9.0 \times 10^{-21} \mathrm{~g}$ & $0.38(\mathrm{~g})$ & $2.9 \times 10^{-27}$ & $\begin{array}{c}\text { Bayesian } \\
\mathcal{F} \text {-statistic } \\
5 n \text {-vector }\end{array}$ & $\begin{array}{c}1.4 \times 10^{-26} \\
\cdots \\
2.6 \times 10^{-26}\end{array}$ & $\begin{array}{c}6.3 \times 10^{-27} \\
\cdots \\
\cdots\end{array}$ & $\begin{array}{c}1.3 \times 10^{-26} \\
\cdots \\
1.3 \times 10^{-26}\end{array}$ & $\begin{array}{c}2.2 \times 10^{30} \\
\cdots \\
2.2 \times 10^{30}\end{array}$ & $\begin{array}{c}2.9 \times 10^{-8} \\
\cdots \\
2.8 \times 10^{-8}\end{array}$ & $\begin{array}{l}4.6 \\
\ldots \\
4.5\end{array}$ & $\begin{array}{c}-3.8 \\
\ldots \\
0.58\end{array}$ & $\begin{array}{c}-2.2 \\
\ldots \\
0.58\end{array}$ \\
\hline J2229+6114 & 19.4 & $7.8 \times 10^{-14}$ & $3.00(\mathrm{hh})$ & $3.3 \times 10^{-25}$ & $\begin{array}{c}\text { Bayesian } \\
\mathcal{F} \text {-statistic } \\
5 n \text {-vector }\end{array}$ & $\begin{array}{c}3.9(3.7) \times 10^{-25} \\
5.6 \times 10^{-25} \\
\ldots\end{array}$ & $\begin{array}{c}1.2(0.8) \times 10^{-26} \\
2.9 \times 10^{-26} \\
\ldots\end{array}$ & $\begin{array}{l}2.5(1.6) \times 10^{-26} \\
2.1 \times 10^{-26} \\
2.5(1.9) \times 10^{-26}\end{array}$ & $\begin{array}{c}3.7(2.3) \times 10^{33} \\
3.1 \times 10^{33} \\
3.7(2.8) \times 10^{33}\end{array}$ & $\begin{array}{c}4.8(3.0) \times 10^{-5} \\
4.0 \times 10^{-5} \\
4.8(3.6) \times 10^{-5}\end{array}$ & $\begin{array}{c}0.077(0.048) \\
0.063 \\
0.077(0.057)\end{array}$ & $\begin{array}{c}-5.0(-5.1) \\
0.55 \\
\ldots\end{array}$ & $\begin{array}{c}-2.8(-2.9) \\
0.43 \\
0.99\end{array}$ \\
\hline $\mathrm{J} 2302+4442^{\mathrm{c}}$ & 192.6 & $1.4 \times 10^{-20}$ & 0.86 (b) & $1.5 \times 10^{-27}$ & $\begin{array}{c}\text { Bayesian } \\
\mathcal{F} \text {-statistic } \\
5 n \text {-vector }\end{array}$ & $\begin{array}{c}1.5 \times 10^{-26} \\
2.5 \times 10^{-26} \\
\ldots\end{array}$ & $\begin{array}{c}6.5 \times 10^{-27} \\
5.6 \times 10^{-27} \\
\quad \ldots\end{array}$ & $\begin{array}{c}1.4 \times 10^{-26} \\
1.1 \times 10^{-26} \\
\quad \cdots\end{array}$ & $\begin{array}{c}5.7 \times 10^{30} \\
4.7 \times 10^{30} \\
\quad \ldots\end{array}$ & $\begin{array}{c}7.4 \times 10^{-8} \\
6.0 \times 10^{-8} \\
\ldots\end{array}$ & $\begin{array}{l}8.9 \\
7.2 \\
\ldots\end{array}$ & $\begin{array}{c}-3.9 \\
0.49 \\
\ldots\end{array}$ & $\begin{array}{c}-2.0 \\
0.49 \\
\ldots\end{array}$ \\
\hline
\end{tabular}

Notes. For references and other notes see Table 2. Values in parentheses are those produced using the restricted orientation priors described in Section 2.2.4.

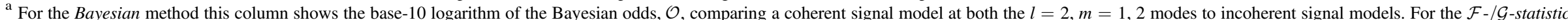

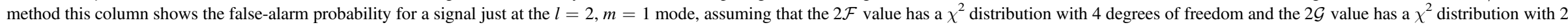

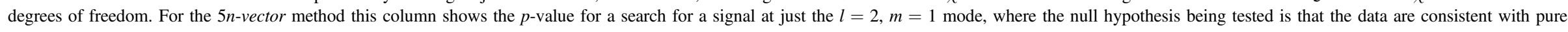
Gaussian noise.

${ }^{\mathrm{b}}$ This is the same as in footnote a, but for all the methods the assumed signal model is from the $l=m=2$ mode.

${ }^{c}$ The observed $\dot{P}$ has been corrected to account for the relative motion between the pulsar and observer.

(This table is available in its entirety in machine-readable form.) 
Table 2

Limits on Gravitational-wave Amplitude and Other Derived Quantities for 188 Pulsars from the Bayesian Analysis

\begin{tabular}{|c|c|c|c|c|c|c|c|c|c|c|c|c|}
\hline $\begin{array}{l}\text { Pulsar Name } \\
\text { (J2000) }\end{array}$ & $\begin{array}{l}f_{\text {rot }} \\
(\mathrm{Hz})\end{array}$ & $\underset{\left(\mathrm{s} \mathrm{s}^{-1}\right)}{\dot{P}_{\text {ot }}}$ & $\begin{array}{l}\text { Distance } \\
\quad(\mathrm{kpc})\end{array}$ & $h_{0}^{\text {sd }}$ & $C_{21}^{95 \%}$ & $C_{22}^{95 \%}$ & $h_{0}^{95 \%}$ & $\begin{array}{c}Q_{22}^{95 \%} \\
\left(\mathrm{~kg} \mathrm{~m}^{2}\right)\end{array}$ & $\varepsilon^{95 \%}$ & $h_{0}^{95 \%} / h_{0}^{\text {sd }}$ & $\mathcal{O}_{m=1,2}^{l=2}$ & $\mathcal{O}_{m=2}^{l=2}$ \\
\hline $\mathrm{J} 0023+0923^{\mathrm{a}}$ & 327.8 & $1.0 \times 10^{-20}$ & 1.10 (a) & $1.3 \times 10^{-27}$ & $2.4 \times 10^{-26}$ & $6.8 \times 10^{-27}$ & $1.5 \times 10^{-26}$ & $2.8 \times 10^{30}$ & $3.6 \times 10^{-8}$ & 11 & -3.9 & -2.2 \\
\hline $\mathrm{J} 0034-0534^{\mathrm{a}}$ & 532.7 & $4.2 \times 10^{-21}$ & 1.35 (b) & $8.9 \times 10^{-28}$ & $2.0 \times 10^{-26}$ & $1.2 \times 10^{-26}$ & $2.5 \times 10^{-26}$ & $2.2 \times 10^{30}$ & $2.8 \times 10^{-8}$ & 28 & -4.1 & -2.1 \\
\hline $\mathrm{J} 0101-6422^{\mathrm{a}}$ & 388.6 & $3.8 \times 10^{-21}$ & 1.00 (b) & $9.7 \times 10^{-28}$ & $2.3 \times 10^{-26}$ & $6.2 \times 10^{-27}$ & $1.3 \times 10^{-26}$ & $1.6 \times 10^{30}$ & $2.1 \times 10^{-8}$ & 14 & -4.1 & -2.3 \\
\hline $\mathrm{J} 0102+4839$ & 337.4 & $1.1 \times 10^{-20}$ & 2.38 (b) & $6.6 \times 10^{-28}$ & $1.9 \times 10^{-26}$ & $9.8 \times 10^{-27}$ & $2.0 \times 10^{-26}$ & $7.6 \times 10^{30}$ & $9.8 \times 10^{-8}$ & 30 & -4.0 & -1.9 \\
\hline $\mathrm{J} 0218+4232^{\mathrm{a}}$ & 430.5 & $7.7 \times 10^{-20}$ & 3.15 (d) & $1.5 \times 10^{-27}$ & $3.1 \times 10^{-26}$ & $1.7 \times 10^{-26}$ & $3.3 \times 10^{-26}$ & $1.0 \times 10^{31}$ & $1.3 \times 10^{-7}$ & 22 & -3.0 & -1.7 \\
\hline J0248+4230 & 384.5 & $1.7 \times 10^{-20}$ & 1.85 (b) & $1.1 \times 10^{-27}$ & $2.6 \times 10^{-26}$ & $1.8 \times 10^{-26}$ & $3.2 \times 10^{-26}$ & $7.4 \times 10^{30}$ & $9.5 \times 10^{-8}$ & 29 & -3.4 & -1.8 \\
\hline J0251+26 & 393.5 & $7.6 \times 10^{-21}$ & 1.15 (b) & $1.2 \times 10^{-27}$ & $2.0 \times 10^{-26}$ & $8.4 \times 10^{-27}$ & $1.8 \times 10^{-26}$ & $2.4 \times 10^{30}$ & $3.1 \times 10^{-8}$ & 15 & -4.0 & -2.1 \\
\hline $\mathrm{J} 0308+74$ & 316.8 & $1.7 \times 10^{-20}$ & 0.38 (b) & $5.0 \times 10^{-27}$ & $1.7 \times 10^{-26}$ & $6.9 \times 10^{-27}$ & $1.5 \times 10^{-26}$ & $1.0 \times 10^{30}$ & $1.3 \times 10^{-8}$ & 3 & -3.9 & -2.2 \\
\hline $\mathrm{J} 0340+4130^{\mathrm{a}}$ & 303.1 & $6.7 \times 10^{-21}$ & 1.60 (b) & $7.2 \times 10^{-28}$ & $2.9 \times 10^{-26}$ & $7.8 \times 10^{-27}$ & $1.7 \times 10^{-26}$ & $5.3 \times 10^{30}$ & $6.8 \times 10^{-8}$ & 23 & -3.5 & -2.1 \\
\hline $\mathrm{J} 0348+0432^{\mathrm{a}}$ & 25.6 & $2.3 \times 10^{-19}$ & $2.10(\mathrm{e})$ & $9.3 \times 10^{-28}$ & $1.4 \times 10^{-25}$ & $8.8 \times 10^{-27}$ & $1.8 \times 10^{-26}$ & $1.1 \times 10^{33}$ & $1.4 \times 10^{-5}$ & 20 & -4.9 & -2.6 \\
\hline J0359+5414 & 12.6 & $1.7 \times 10^{-14}$ & $\ldots$ & $\cdots$ & $7.9 \times 10^{-24}$ & $4.0 \times 10^{-26}$ & $8.6 \times 10^{-26}$ & & $\ldots$ & $\ldots$ & -4.8 & -2.7 \\
\hline $\mathrm{J} 0407+1607$ & 38.9 & $7.9 \times 10^{-20}$ & 1.34 (b) & $1.1 \times 10^{-27}$ & $4.8 \times 10^{-26}$ & $5.3 \times 10^{-27}$ & $1.1 \times 10^{-26}$ & $1.8 \times 10^{32}$ & $2.4 \times 10^{-6}$ & 11 & -4.7 & -2.4 \\
\hline $\mathrm{J} 0437-4715^{\mathrm{a}}$ & 173.7 & $1.4 \times 10^{-20}$ & 0.16 (f) & $7.9 \times 10^{-27}$ & $1.5 \times 10^{-26}$ & $8.3 \times 10^{-27}$ & $1.6 \times 10^{-26}$ & $1.5 \times 10^{30}$ & $2.0 \times 10^{-8}$ & 2 & -4.4 & -2.5 \\
\hline $\mathrm{J} 0453+1559^{\mathrm{a}}$ & 21.8 & $1.8 \times 10^{-19}$ & 0.52 (b) & $3.1 \times 10^{-27}$ & $1.9 \times 10^{-25}$ & $9.2 \times 10^{-27}$ & $2.1 \times 10^{-26}$ & $4.1 \times 10^{32}$ & $5.3 \times 10^{-6}$ & 6.6 & -5.2 & -2.8 \\
\hline $\mathrm{J} 0533+67$ & 227.9 & $1.3 \times 10^{-20}$ & $2.28(\mathrm{~b})$ & $6.0 \times 10^{-28}$ & $1.4 \times 10^{-26}$ & $6.7 \times 10^{-27}$ & $1.4 \times 10^{-26}$ & $1.1 \times 10^{31}$ & $1.5 \times 10^{-7}$ & 24 & -3.9 & -2.0 \\
\hline $\mathrm{J} 0557+1550$ & 391.2 & $7.4 \times 10^{-21}$ & 1.83 (b) & $7.5 \times 10^{-28}$ & $1.7 \times 10^{-26}$ & $1.0 \times 10^{-26}$ & $2.1 \times 10^{-26}$ & $4.7 \times 10^{30}$ & $6.1 \times 10^{-8}$ & 29 & -4.0 & -2.0 \\
\hline J0605+37 & 366.6 & $4.7 \times 10^{-21}$ & 0.19 (b) & $5.6 \times 10^{-27}$ & $2.3 \times 10^{-26}$ & $1.6 \times 10^{-26}$ & $3.1 \times 10^{-26}$ & $8.0 \times 10^{29}$ & $1.0 \times 10^{-8}$ & 5.6 & -3.0 & -1.3 \\
\hline J0609+2130 & 18.0 & $2.4 \times 10^{-19}$ & 0.57 (b) & $2.9 \times 10^{-27}$ & $8.9 \times 10^{-25}$ & $1.9 \times 10^{-26}$ & $3.9 \times 10^{-26}$ & $1.3 \times 10^{33}$ & $1.6 \times 10^{-5}$ & 13 & -4.6 & -2.6 \\
\hline $\mathrm{J} 0610-2100^{\mathrm{a}}$ & 259.0 & $1.1 \times 10^{-21}$ & $3.26(\mathrm{~b})$ & $1.3 \times 10^{-28}$ & $1.7 \times 10^{-26}$ & $6.0 \times 10^{-27}$ & $1.3 \times 10^{-26}$ & $1.2 \times 10^{31}$ & $1.5 \times 10^{-7}$ & 99 & -4.0 & -2.2 \\
\hline J0613-0200 & 326.6 & $8.9 \times 10^{-21}(\mathrm{~g})$ & $0.78(\mathrm{~g})$ & $1.8 \times 10^{-27}$ & $1.7 \times 10^{-26}$ & $1.1 \times 10^{-26}$ & $2.3 \times 10^{-26}$ & $3.1 \times 10^{30}$ & $4.0 \times 10^{-8}$ & 13 & -3.9 & -1.9 \\
\hline $\mathrm{J} 0614-3329^{\mathrm{a}}$ & 317.6 & $1.8 \times 10^{-20}$ & $0.63(\mathrm{~h})$ & $3.0 \times 10^{-27}$ & $2.4 \times 10^{-26}$ & $1.0 \times 10^{-26}$ & $1.9 \times 10^{-26}$ & $2.1 \times 10^{30}$ & $2.8 \times 10^{-8}$ & 6.2 & -3.8 & -2.0 \\
\hline $\mathrm{J} 0621+1002^{\mathrm{a}}$ & 34.7 & $4.6 \times 10^{-20}$ & 0.42 (b) & $2.4 \times 10^{-27}$ & $7.0 \times 10^{-26}$ & $7.7 \times 10^{-27}$ & $1.6 \times 10^{-26}$ & $1.0 \times 10^{32}$ & $1.3 \times 10^{-6}$ & 6.6 & -4.6 & -2.3 \\
\hline $\mathrm{J} 0621+25$ & 367.4 & $2.5 \times 10^{-20}$ & $1.64(\mathrm{~b})$ & $1.5 \times 10^{-27}$ & $2.6 \times 10^{-26}$ & $1.1 \times 10^{-26}$ & $2.5 \times 10^{-26}$ & $5.5 \times 10^{30}$ & $7.1 \times 10^{-8}$ & 17 & -3.7 & -1.9 \\
\hline $\mathrm{J} 0636+5129^{\mathrm{a}}$ & 348.6 & $3.4 \times 10^{-21}$ & 0.21 (b) & $4.2 \times 10^{-27}$ & $1.6 \times 10^{-26}$ & $6.2 \times 10^{-27}$ & $1.4 \times 10^{-26}$ & $4.5 \times 10^{29}$ & $5.8 \times 10^{-9}$ & 3.4 & -4.8 & -2.3 \\
\hline $\mathrm{J} 0645+5158^{\mathrm{a}}$ & 112.9 & $3.6 \times 10^{-21}$ & 1.20 (a) & $4.3 \times 10^{-28}$ & $1.7 \times 10^{-26}$ & $8.5 \times 10^{-27}$ & $1.7 \times 10^{-26}$ & $2.9 \times 10^{31}$ & $3.8 \times 10^{-7}$ & 39 & -3.4 & -1.5 \\
\hline J0721-2038 & 64.3 & $4.4 \times 10^{-20}$ & $2.68(\mathrm{~b})$ & $5.1 \times 10^{-28}$ & $3.2 \times 10^{-26}$ & $7.4 \times 10^{-27}$ & $1.5 \times 10^{-26}$ & $1.7 \times 10^{32}$ & $2.2 \times 10^{-6}$ & 29 & -3.6 & -1.6 \\
\hline $\mathrm{J} 0737-3039 \mathrm{~A}^{\mathrm{a}}$ & 44.1 & $1.8 \times 10^{-18}$ & 1.10 (i) & $6.5 \times 10^{-27}$ & $5.1 \times 10^{-26}$ & $5.2 \times 10^{-27}$ & $1.1 \times 10^{-26}$ & $1.2 \times 10^{32}$ & $1.5 \times 10^{-6}$ & 1.7 & -4.3 & -2.3 \\
\hline $\mathrm{J} 0740+6620^{\mathrm{a}}$ & 346.5 & $8.6 \times 10^{-21}$ & 0.40 (a) & $3.5 \times 10^{-27}$ & $1.6 \times 10^{-26}$ & $7.9 \times 10^{-27}$ & $1.6 \times 10^{-26}$ & $9.9 \times 10^{29}$ & $1.3 \times 10^{-8}$ & 4.7 & -4.9 & -2.3 \\
\hline J0751+1807 & 287.5 & $6.2 \times 10^{-21}(\mathrm{~g})$ & $1.00(\mathrm{~g})$ & $1.1 \times 10^{-27}$ & $1.6 \times 10^{-26}$ & $5.7 \times 10^{-27}$ & $1.3 \times 10^{-26}$ & $2.8 \times 10^{30}$ & $3.6 \times 10^{-8}$ & 12 & -4.1 & -2.2 \\
\hline J0900-3144 & 90.0 & $5.0 \times 10^{-20}(\mathrm{~g})$ & $0.81(\mathrm{~g})$ & $2.1 \times 10^{-27}$ & $1.6 \times 10^{-26}$ & $5.0 \times 10^{-27}$ & $1.1 \times 10^{-26}$ & $2.0 \times 10^{31}$ & $2.6 \times 10^{-7}$ & 5.1 & -5.0 & -2.8 \\
\hline $\mathrm{J} 0931-1902^{\mathrm{a}}$ & 215.6 & $3.2 \times 10^{-21}$ & 3.72 (b) & $1.8 \times 10^{-28}$ & $1.6 \times 10^{-26}$ & $5.8 \times 10^{-27}$ & $1.3 \times 10^{-26}$ & $1.9 \times 10^{31}$ & $2.4 \times 10^{-7}$ & 71 & -3.9 & -2.1 \\
\hline J0952-0607 & 707.3 & $4.8 \times 10^{-21}$ & $1.74(\mathrm{~b})$ & $8.5 \times 10^{-28}$ & $5.5 \times 10^{-26}$ & $2.7 \times 10^{-26}$ & $5.5 \times 10^{-26}$ & $3.5 \times 10^{30}$ & $4.5 \times 10^{-8}$ & 65 & -2.1 & -1.1 \\
\hline J0955-61 & 500.2 & $1.4 \times 10^{-20}$ & 2.17 (b) & $9.9 \times 10^{-28}$ & $3.8 \times 10^{-26}$ & $1.2 \times 10^{-26}$ & $2.6 \times 10^{-26}$ & $4.1 \times 10^{30}$ & $5.3 \times 10^{-8}$ & 26 & -3.6 & -2.1 \\
\hline $\mathrm{J} 1012+5307$ & 190.3 & $8.0 \times 10^{-21}(\mathrm{~g})$ & $1.11(\mathrm{k})$ & $9.0 \times 10^{-28}$ & $1.6 \times 10^{-26}$ & $6.5 \times 10^{-27}$ & $1.3 \times 10^{-26}$ & $7.5 \times 10^{30}$ & $9.7 \times 10^{-8}$ & 15 & -3.9 & -2.0 \\
\hline $\mathrm{J} 1012-4235$ & 322.5 & $6.6 \times 10^{-21}$ & 0.37 (b) & $3.2 \times 10^{-27}$ & $1.6 \times 10^{-26}$ & $8.9 \times 10^{-27}$ & $1.8 \times 10^{-26}$ & $1.2 \times 10^{30}$ & $1.5 \times 10^{-8}$ & 5.7 & -3.9 & -1.9 \\
\hline J1017-7156 & 427.6 & $1.2 \times 10^{-21}(\mathrm{kk})$ & 0.70 (1) & $8.3 \times 10^{-28}$ & $1.7 \times 10^{-26}$ & $8.9 \times 10^{-27}$ & $1.9 \times 10^{-26}$ & $1.3 \times 10^{30}$ & $1.7 \times 10^{-8}$ & 23 & -4.2 & -2.2 \\
\hline $\mathrm{J} 1022+1001$ & 60.8 & $3.0 \times 10^{-20}(\mathrm{~g})$ & $1.09(\mathrm{~g})$ & $1.0 \times 10^{-27}$ & $3.5 \times 10^{-26}$ & $5.8 \times 10^{-27}$ & $1.2 \times 10^{-26}$ & $6.5 \times 10^{31}$ & $8.4 \times 10^{-7}$ & 12 & -4.0 & -2.0 \\
\hline $\mathrm{J} 1024-0719^{\mathrm{b}}$ & 193.7 & $\ldots$ & $1.08(\mathrm{~g})$ & $\ldots$ & $1.7 \times 10^{-26}$ & $8.5 \times 10^{-27}$ & $1.7 \times 10^{-26}$ & $9.0 \times 10^{30}$ & $1.2 \times 10^{-7}$ & $\cdots$ & -3.7 & -1.9 \\
\hline $\mathrm{J} 1035-6720^{\mathrm{b}}$ & 348.2 & $\ldots$ & 1.46 (b) & $\ldots$ & $1.9 \times 10^{-26}$ & $6.8 \times 10^{-27}$ & $1.5 \times 10^{-26}$ & $3.2 \times 10^{30}$ & $4.2 \times 10^{-8}$ & $\ldots$ & -4.7 & -2.3 \\
\hline J1036-8317 & 293.4 & $3.1 \times 10^{-20}$ & 0.93 (b) & $2.6 \times 10^{-27}$ & $2.2 \times 10^{-26}$ & $8.1 \times 10^{-27}$ & $1.7 \times 10^{-26}$ & $3.4 \times 10^{30}$ & $4.4 \times 10^{-8}$ & 6.6 & -3.7 & -2.0 \\
\hline $\mathrm{J} 1038+0032$ & 34.7 & $6.7 \times 10^{-20}$ & 5.94 (b) & $2.1 \times 10^{-28}$ & $6.5 \times 10^{-26}$ & $6.6 \times 10^{-27}$ & $1.4 \times 10^{-26}$ & $1.3 \times 10^{33}$ & $1.6 \times 10^{-5}$ & 68 & -4.7 & -2.4 \\
\hline $\mathrm{J} 1055-6028$ & 10.0 & $3.0 \times 10^{-14}$ & 3.83 (b) & $1.1 \times 10^{-25}$ & $8.4 \times 10^{-23}$ & $1.2 \times 10^{-25}$ & $2.0 \times 10^{-25}$ & $1.4 \times 10^{35}$ & $1.8 \times 10^{-3}$ & 1.8 & -1.8 & -3.0 \\
\hline J1124-3653 & 415.0 & $6.0 \times 10^{-21}$ & 1.05 (b) & $1.2 \times 10^{-27}$ & $3.1 \times 10^{-26}$ & $6.9 \times 10^{-27}$ & $1.6 \times 10^{-26}$ & $1.8 \times 10^{30}$ & $2.4 \times 10^{-8}$ & 14 & -3.7 & -2.2 \\
\hline $\mathrm{J} 1125+7819^{\mathrm{b}}$ & 238.0 & $\ldots$ & 0.88 (b) & $\ldots$ & $2.1 \times 10^{-26}$ & $4.7 \times 10^{-27}$ & $1.0 \times 10^{-26}$ & $2.9 \times 10^{30}$ & $3.7 \times 10^{-8}$ & $\cdots$ & -3.8 & -2.2 \\
\hline J1125-5825 & 322.4 & $5.9 \times 10^{-20}(\mathrm{kk})$ & 1.74 (b) & $2.0 \times 10^{-27}$ & $2.0 \times 10^{-26}$ & $1.0 \times 10^{-26}$ & $2.0 \times 10^{-26}$ & $6.1 \times 10^{30}$ & $7.8 \times 10^{-8}$ & 9.8 & -3.8 & -1.9 \\
\hline $\mathrm{J} 1137+7528$ & 398.0 & $3.2 \times 10^{-21}$ & 3.81 (b) & $2.4 \times 10^{-28}$ & $2.4 \times 10^{-26}$ & $7.8 \times 10^{-27}$ & $1.6 \times 10^{-26}$ & $7.1 \times 10^{30}$ & $9.2 \times 10^{-8}$ & 67 & -3.8 & -2.2 \\
\hline $\mathrm{J} 1142+0119$ & 197.0 & $1.5 \times 10^{-20}$ & 2.18 (b) & $6.4 \times 10^{-28}$ & $3.1 \times 10^{-26}$ & $1.0 \times 10^{-26}$ & $2.4 \times 10^{-26}$ & $2.5 \times 10^{31}$ & $3.2 \times 10^{-7}$ & 38 & -2.8 & -1.3 \\
\hline
\end{tabular}


Table 2

(Continued)

\begin{tabular}{|c|c|c|c|c|c|c|c|c|c|c|c|c|}
\hline $\begin{array}{l}\text { Pulsar Name } \\
(\mathrm{J} 2000)\end{array}$ & $\begin{array}{c}f_{\text {rot }} \\
(\mathrm{Hz})\end{array}$ & $\underset{\left(\mathrm{s} \mathrm{s}^{-1}\right)}{\dot{P}_{\text {ot }}}$ & $\begin{array}{l}\text { Distance } \\
\quad(\mathrm{kpc})\end{array}$ & $h_{0}^{\text {sd }}$ & $C_{21}^{95 \%}$ & $C_{22}^{95 \%}$ & $h_{0}^{95 \%}$ & $\begin{array}{c}Q_{22}^{95 \%} \\
\left(\mathrm{~kg} \mathrm{~m}^{2}\right)\end{array}$ & $\varepsilon^{95 \%}$ & $h_{0}^{95 \%} / h_{0}^{\text {sd }}$ & $\mathcal{O}_{m=1,2}^{l=2}$ & $\mathcal{O}_{m=2}^{l=2}$ \\
\hline J1207-5050 & 206.5 & $6.1 \times 10^{-21}$ & 1.27 (b) & $7.1 \times 10^{-28}$ & $1.5 \times 10^{-26}$ & $5.4 \times 10^{-27}$ & $1.1 \times 10^{-26}$ & $6.1 \times 10^{30}$ & $7.9 \times 10^{-8}$ & 16 & -3.9 & -2.1 \\
\hline $\mathrm{J} 1231-1411^{\mathrm{a}}$ & 271.5 & $8.2 \times 10^{-21}$ & 0.42 (b) & $2.9 \times 10^{-27}$ & $1.9 \times 10^{-26}$ & $7.9 \times 10^{-27}$ & $1.7 \times 10^{-26}$ & $1.7 \times 10^{30}$ & $2.3 \times 10^{-8}$ & 5.8 & -3.7 & -1.9 \\
\hline $\mathrm{J} 1300+1240^{\mathrm{a}}$ & 160.8 & $3.1 \times 10^{-20}$ & $0.60(\mathrm{~m})$ & $3.0 \times 10^{-27}$ & $2.3 \times 10^{-26}$ & $5.5 \times 10^{-27}$ & $1.2 \times 10^{-26}$ & $5.2 \times 10^{30}$ & $6.7 \times 10^{-8}$ & 4.1 & -3.7 & -2.1 \\
\hline $\mathrm{J} 1301+0833$ & 542.4 & $1.1 \times 10^{-20}$ & $1.23(\mathrm{~b})$ & $1.6 \times 10^{-27}$ & $2.7 \times 10^{-26}$ & $2.0 \times 10^{-26}$ & $4.3 \times 10^{-26}$ & $3.3 \times 10^{30}$ & $4.3 \times 10^{-8}$ & 28 & -3.6 & -1.9 \\
\hline $\mathrm{J} 1302-32$ & 265.2 & $6.6 \times 10^{-21}$ & 1.49 (b) & $7.1 \times 10^{-28}$ & $2.0 \times 10^{-26}$ & $6.2 \times 10^{-27}$ & $1.3 \times 10^{-26}$ & $4.9 \times 10^{30}$ & $6.3 \times 10^{-8}$ & 18 & -3.9 & -2.2 \\
\hline $\mathrm{J} 1311-3430$ & 390.6 & $2.1 \times 10^{-20}$ & $2.43(\mathrm{~b})$ & $9.5 \times 10^{-28}$ & $1.8 \times 10^{-26}$ & $1.3 \times 10^{-26}$ & $2.8 \times 10^{-26}$ & $8.0 \times 10^{30}$ & $1.0 \times 10^{-7}$ & 29 & -3.7 & -1.7 \\
\hline $\mathrm{J} 1312+0051$ & 236.5 & $1.8 \times 10^{-20}$ & $1.47(\mathrm{~b})$ & $1.1 \times 10^{-27}$ & $1.9 \times 10^{-26}$ & $6.8 \times 10^{-27}$ & $1.4 \times 10^{-26}$ & $6.9 \times 10^{30}$ & $8.9 \times 10^{-8}$ & 13 & -3.8 & -2.0 \\
\hline $\mathrm{J} 1327-0755^{\mathrm{b}}$ & 373.4 & $\ldots$ & $1.70(\mathrm{n})$ & $\ldots$ & $1.6 \times 10^{-26}$ & $8.7 \times 10^{-27}$ & $1.8 \times 10^{-26}$ & $4.1 \times 10^{30}$ & $5.3 \times 10^{-8}$ & $\ldots$ & -4.0 & -2.1 \\
\hline J1446-4701 & 455.6 & $9.7 \times 10^{-21}(\mathrm{kk})$ & 1.57 (b) & $1.1 \times 10^{-27}$ & $2.7 \times 10^{-26}$ & $1.4 \times 10^{-26}$ & $2.9 \times 10^{-26}$ & $4.0 \times 10^{30}$ & $5.2 \times 10^{-8}$ & 27 & -3.6 & -1.9 \\
\hline $\mathrm{J} 1453+1902^{\mathrm{a}}$ & 172.6 & $9.1 \times 10^{-21}$ & 1.27 (b) & $8.0 \times 10^{-28}$ & $1.9 \times 10^{-26}$ & $8.3 \times 10^{-27}$ & $1.6 \times 10^{-26}$ & $1.2 \times 10^{31}$ & $1.6 \times 10^{-7}$ & 20 & -4.1 & -2.4 \\
\hline J1455-3330 & 125.2 & $2.3 \times 10^{-20}(\mathrm{~g})$ & $0.80(\mathrm{~g})$ & $1.7 \times 10^{-27}$ & $2.1 \times 10^{-26}$ & $5.2 \times 10^{-27}$ & $1.0 \times 10^{-26}$ & $9.5 \times 10^{30}$ & $1.2 \times 10^{-7}$ & 5.9 & -3.8 & -2.0 \\
\hline $\mathrm{J} 1513-2550$ & 471.9 & $2.1 \times 10^{-20}$ & 3.97 (b) & $6.5 \times 10^{-28}$ & $1.7 \times 10^{-26}$ & $8.6 \times 10^{-27}$ & $1.9 \times 10^{-26}$ & $6.2 \times 10^{30}$ & $8.0 \times 10^{-8}$ & 29 & -4.3 & -2.2 \\
\hline $\mathrm{J} 1514-4946^{\mathrm{a}}$ & 278.6 & $1.2 \times 10^{-20}$ & $0.91(b)$ & $1.6 \times 10^{-27}$ & $1.4 \times 10^{-26}$ & $6.2 \times 10^{-27}$ & $1.4 \times 10^{-26}$ & $2.9 \times 10^{30}$ & $3.8 \times 10^{-8}$ & 8.6 & -4.0 & -2.1 \\
\hline $\mathrm{J} 1518+4904^{\mathrm{a}}$ & 24.4 & $2.3 \times 10^{-20}$ & 0.96 (b) & $6.3 \times 10^{-28}$ & $2.0 \times 10^{-25}$ & $8.2 \times 10^{-27}$ & $1.8 \times 10^{-26}$ & $5.2 \times 10^{32}$ & $6.8 \times 10^{-6}$ & 28 & -4.8 & -2.8 \\
\hline $\mathrm{J} 1528-3146$ & 16.4 & $2.5 \times 10^{-19}$ & 0.77 (b) & $2.1 \times 10^{-27}$ & $1.6 \times 10^{-24}$ & $1.8 \times 10^{-26}$ & $3.7 \times 10^{-26}$ & $1.9 \times 10^{33}$ & $2.5 \times 10^{-5}$ & 18 & -4.5 & -2.6 \\
\hline J1536-4948 & 324.7 & $2.1 \times 10^{-20}$ & 0.98 (b) & $2.2 \times 10^{-27}$ & $2.0 \times 10^{-26}$ & $8.8 \times 10^{-27}$ & $2.0 \times 10^{-26}$ & $3.5 \times 10^{30}$ & $4.5 \times 10^{-8}$ & 9.5 & -3.7 & -2.0 \\
\hline $\mathrm{J} 1537+1155^{\mathrm{a}}$ & 26.4 & $2.4 \times 10^{-18}$ & $1.05(\mathrm{p})$ & $6.1 \times 10^{-27}$ & $1.3 \times 10^{-25}$ & $7.4 \times 10^{-27}$ & $1.6 \times 10^{-26}$ & $4.3 \times 10^{32}$ & $5.5 \times 10^{-6}$ & 2.6 & -4.9 & -2.7 \\
\hline $\mathrm{J} 1544+4937$ & 463.1 & $2.9 \times 10^{-21}$ & 2.99 (b) & $3.1 \times 10^{-28}$ & $1.8 \times 10^{-26}$ & $1.0 \times 10^{-26}$ & $2.2 \times 10^{-26}$ & $5.5 \times 10^{30}$ & $7.1 \times 10^{-8}$ & 69 & -4.0 & -2.1 \\
\hline $\mathrm{J} 1551-0658$ & 141.0 & $2.0 \times 10^{-20}$ & $1.32(\mathrm{~b})$ & $1.0 \times 10^{-27}$ & $2.4 \times 10^{-26}$ & $1.1 \times 10^{-26}$ & $2.1 \times 10^{-26}$ & $2.5 \times 10^{31}$ & $3.3 \times 10^{-7}$ & 20 & -3.0 & -1.5 \\
\hline $\mathrm{J} 1552+5437$ & 411.9 & $2.8 \times 10^{-21}$ & $2.64(\mathrm{~b})$ & $3.3 \times 10^{-28}$ & $2.7 \times 10^{-26}$ & $9.1 \times 10^{-27}$ & $1.8 \times 10^{-26}$ & $5.3 \times 10^{30}$ & $6.8 \times 10^{-8}$ & 56 & -3.5 & -2.1 \\
\hline $\mathrm{J} 1600-3053$ & 277.9 & $8.6 \times 10^{-21}(\mathrm{~g})$ & $1.49(\mathrm{~g})$ & $8.4 \times 10^{-28}$ & $1.8 \times 10^{-26}$ & $6.6 \times 10^{-27}$ & $1.4 \times 10^{-26}$ & $4.9 \times 10^{30}$ & $6.3 \times 10^{-8}$ & 17 & -4.0 & -2.2 \\
\hline $\mathrm{J} 1603-7202^{\mathrm{a}}$ & 67.4 & $1.4 \times 10^{-20}$ & 0.53 (f) & $1.5 \times 10^{-27}$ & $3.3 \times 10^{-26}$ & $5.1 \times 10^{-27}$ & $1.0 \times 10^{-26}$ & $2.1 \times 10^{31}$ & $2.8 \times 10^{-7}$ & 6.7 & -3.7 & -2.1 \\
\hline $\mathrm{J} 1614-2230^{\mathrm{a}}$ & 317.4 & $3.5 \times 10^{-21}$ & 0.67 (a) & $1.3 \times 10^{-27}$ & $1.8 \times 10^{-26}$ & $1.2 \times 10^{-26}$ & $2.4 \times 10^{-26}$ & $2.9 \times 10^{30}$ & $3.8 \times 10^{-8}$ & 19 & -3.4 & -1.6 \\
\hline J1618-3921 & 83.4 & $5.4 \times 10^{-20}$ & $5.52(\mathrm{~b})$ & $3.1 \times 10^{-28}$ & $2.3 \times 10^{-26}$ & $4.2 \times 10^{-27}$ & $9.1 \times 10^{-27}$ & $1.3 \times 10^{32}$ & $1.7 \times 10^{-6}$ & 29 & -4.0 & -2.1 \\
\hline $\mathrm{J} 1623-2631^{\mathrm{c}}$ & 90.3 & $8.8 \times 10^{-20}$ & $1.80(\mathrm{q})$ & $1.3 \times 10^{-27}$ & $2.7 \times 10^{-26}$ & $4.1 \times 10^{-27}$ & $8.9 \times 10^{-27}$ & $3.6 \times 10^{31}$ & $4.6 \times 10^{-7}$ & 7 & -3.7 & -2.1 \\
\hline $\mathrm{J} 1623-5005$ & 11.8 & $4.2 \times 10^{-15}$ & $\ldots$ & $\ldots$ & $1.0 \times 10^{-23}$ & $7.4 \times 10^{-26}$ & $1.5 \times 10^{-25}$ & $\ldots$ & & $\ldots$ & -3.9 & -2.3 \\
\hline J1628-3205 & 311.4 & $1.3 \times 10^{-20}$ & $1.22(\mathrm{~b})$ & $1.3 \times 10^{-27}$ & $1.6 \times 10^{-26}$ & $8.4 \times 10^{-27}$ & $1.7 \times 10^{-26}$ & $4.0 \times 10^{30}$ & $5.2 \times 10^{-8}$ & 13 & -4.0 & -2.1 \\
\hline $\mathrm{J} 1630+37$ & 301.4 & $1.1 \times 10^{-20}$ & 1.18 (b) & $1.2 \times 10^{-27}$ & $1.6 \times 10^{-26}$ & $1.6 \times 10^{-26}$ & $3.3 \times 10^{-26}$ & $7.7 \times 10^{30}$ & $1.0 \times 10^{-7}$ & 27 & -3.3 & -1.4 \\
\hline $\mathrm{J} 1640+2224^{\mathrm{a}}$ & 316.1 & $1.3 \times 10^{-21}$ & $1.52(\mathrm{r})$ & $3.4 \times 10^{-28}$ & $2.6 \times 10^{-26}$ & $9.9 \times 10^{-27}$ & $1.9 \times 10^{-26}$ & $5.3 \times 10^{30}$ & $6.9 \times 10^{-8}$ & 57 & -3.5 & -2.0 \\
\hline $\mathrm{J} 1643-1224$ & 216.4 & $1.8 \times 10^{-20}(\mathrm{~g})$ & $0.76(\mathrm{~g})$ & $2.1 \times 10^{-27}$ & $1.8 \times 10^{-26}$ & $5.9 \times 10^{-27}$ & $1.2 \times 10^{-26}$ & $3.7 \times 10^{30}$ & $4.8 \times 10^{-8}$ & 5.9 & -3.9 & -2.1 \\
\hline J1653-2054 & 242.2 & $1.1 \times 10^{-20}$ & $2.63(\mathrm{~b})$ & $5.0 \times 10^{-28}$ & $1.5 \times 10^{-26}$ & $6.1 \times 10^{-27}$ & $1.3 \times 10^{-26}$ & $1.1 \times 10^{31}$ & $1.4 \times 10^{-7}$ & 26 & -3.9 & -2.1 \\
\hline $\mathrm{J} 1658-5324^{\mathrm{a}}$ & 410.0 & $1.1 \times 10^{-20}$ & 0.88 (b) & $1.9 \times 10^{-27}$ & $1.4 \times 10^{-26}$ & $2.4 \times 10^{-26}$ & $4.9 \times 10^{-26}$ & $4.7 \times 10^{30}$ & $6.0 \times 10^{-8}$ & 25 & -2.6 & -0.7 \\
\hline $\mathrm{J} 1710+49$ & 310.5 & $1.8 \times 10^{-20}$ & $0.51(\mathrm{~b})$ & $3.8 \times 10^{-27}$ & $2.0 \times 10^{-26}$ & $5.6 \times 10^{-27}$ & $1.2 \times 10^{-26}$ & $1.2 \times 10^{30}$ & $1.6 \times 10^{-8}$ & 3.3 & -4.1 & -2.3 \\
\hline $\mathrm{J} 1713+0747$ & 218.8 & $8.1 \times 10^{-21}(\mathrm{~g})$ & $1.11(\mathrm{~g})$ & $9.7 \times 10^{-28}$ & $1.8 \times 10^{-26}$ & $8.4 \times 10^{-27}$ & $1.7 \times 10^{-26}$ & $7.0 \times 10^{30}$ & $9.1 \times 10^{-8}$ & 17 & -3.5 & -1.8 \\
\hline $\mathrm{J} 1719-1438^{\mathrm{b}}$ & 172.7 & $\ldots$ & 0.34 (b) & $\ldots$ & $1.7 \times 10^{-26}$ & $7.4 \times 10^{-27}$ & $1.5 \times 10^{-26}$ & $3.1 \times 10^{30}$ & $4.0 \times 10^{-8}$ & $\ldots$ & -4.3 & -2.5 \\
\hline $\mathrm{J} 1721-2457^{\mathrm{b}}$ & 286.0 & $\ldots$ & $1.37(\mathrm{~b})$ & $\ldots$ & $1.6 \times 10^{-26}$ & $7.2 \times 10^{-27}$ & $1.5 \times 10^{-26}$ & $4.7 \times 10^{30}$ & $6.0 \times 10^{-8}$ & $\ldots$ & -4.0 & -2.1 \\
\hline$J 1727-2946^{\mathrm{a}}$ & 36.9 & $2.4 \times 10^{-19}$ & $1.88(\mathrm{~b})$ & $1.3 \times 10^{-27}$ & $1.0 \times 10^{-25}$ & $8.0 \times 10^{-27}$ & $1.8 \times 10^{-26}$ & $4.6 \times 10^{32}$ & $5.9 \times 10^{-6}$ & 14 & -4.0 & -2.2 \\
\hline J1729-2117 & 15.1 & $1.7 \times 10^{-19}$ & 0.97 (b) & $1.3 \times 10^{-27}$ & $2.0 \times 10^{-24}$ & $3.7 \times 10^{-26}$ & $7.6 \times 10^{-26}$ & $5.9 \times 10^{33}$ & $7.7 \times 10^{-5}$ & 57 & -4.1 & -2.1 \\
\hline J1730-2304 & 123.1 & $1.0 \times 10^{-20}(\mathrm{~g})$ & $0.90(\mathrm{~g})$ & $9.9 \times 10^{-28}$ & $2.0 \times 10^{-26}$ & $4.4 \times 10^{-27}$ & $9.3 \times 10^{-27}$ & $1.0 \times 10^{31}$ & $1.3 \times 10^{-7}$ & 9.4 & -3.8 & -2.1 \\
\hline $\mathrm{J} 1732-5049^{\mathrm{a}}$ & 188.2 & $1.2 \times 10^{-20}$ & $4.22(\mathrm{~s})$ & $2.8 \times 10^{-28}$ & $1.4 \times 10^{-26}$ & $5.0 \times 10^{-27}$ & $1.1 \times 10^{-26}$ & $2.3 \times 10^{31}$ & $3.0 \times 10^{-7}$ & 37 & -4.1 & -2.2 \\
\hline $\mathrm{J} 1738+0333$ & 170.9 & $2.2 \times 10^{-20}(\mathrm{t})$ & $1.47(\mathrm{t})$ & $1.1 \times 10^{-27}$ & $1.5 \times 10^{-26}$ & $4.8 \times 10^{-27}$ & $1.0 \times 10^{-26}$ & $9.3 \times 10^{30}$ & $1.2 \times 10^{-7}$ & 9.5 & -4.6 & -2.7 \\
\hline $\mathrm{J} 1741+1351^{\mathrm{a}}$ & 266.9 & $2.9 \times 10^{-20}$ & $1.08(\mathrm{u})$ & $2.1 \times 10^{-27}$ & $2.0 \times 10^{-26}$ & $1.1 \times 10^{-26}$ & $2.2 \times 10^{-26}$ & $6.0 \times 10^{30}$ & $7.8 \times 10^{-8}$ & 11 & -3.3 & -1.5 \\
\hline J1744-1134 & 245.4 & $7.0 \times 10^{-21}(\mathrm{~g})$ & $0.42(\mathrm{~g})$ & $2.5 \times 10^{-27}$ & $2.1 \times 10^{-26}$ & $1.3 \times 10^{-26}$ & $2.5 \times 10^{-26}$ & $3.2 \times 10^{30}$ & $4.1 \times 10^{-8}$ & 10 & -2.7 & -1.1 \\
\hline $\mathrm{J} 1744-7619^{\mathrm{b}}$ & 213.3 & $\ldots$ & $\ldots$ & $\ldots$ & $1.3 \times 10^{-26}$ & $6.6 \times 10^{-27}$ & $1.4 \times 10^{-26}$ & $\ldots$ & $\ldots$ & $\cdots$ & -4.0 & -2.0 \\
\hline $\mathrm{J} 1745+1017^{\mathrm{a}}$ & 377.1 & $2.2 \times 10^{-21}$ & $1.21(\mathrm{~b})$ & $6.0 \times 10^{-28}$ & $1.6 \times 10^{-26}$ & $7.4 \times 10^{-27}$ & $1.6 \times 10^{-26}$ & $2.5 \times 10^{30}$ & $3.3 \times 10^{-8}$ & 27 & -4.1 & -2.3 \\
\hline $\mathrm{J} 1747-4036^{\mathrm{a}}$ & 607.7 & $1.1 \times 10^{-20}$ & 7.15 (b) & $2.9 \times 10^{-28}$ & $2.9 \times 10^{-26}$ & $1.2 \times 10^{-26}$ & $2.6 \times 10^{-26}$ & $9.3 \times 10^{30}$ & $1.2 \times 10^{-7}$ & 90 & -3.9 & -2.1 \\
\hline $\mathrm{J} 1748-2446 \mathrm{~A}^{\mathrm{c}}$ & 86.5 & $9.2 \times 10^{-20}$ & $5.50(\mathrm{v})$ & $4.1 \times 10^{-28}$ & $2.1 \times 10^{-26}$ & $6.9 \times 10^{-27}$ & $1.4 \times 10^{-26}$ & $1.8 \times 10^{32}$ & $2.4 \times 10^{-6}$ & 33 & -3.8 & -1.8 \\
\hline
\end{tabular}


Table 2

(Continued)

\begin{tabular}{|c|c|c|c|c|c|c|c|c|c|c|c|c|}
\hline $\begin{array}{l}\text { Pulsar Name } \\
\text { (J2000) }\end{array}$ & $\begin{array}{l}f_{\text {rot }} \\
(\mathrm{Hz})\end{array}$ & $\underset{\left(\mathrm{s} \mathrm{s}^{-1}\right)}{\dot{P}_{\text {ot }}}$ & $\begin{array}{l}\text { Distance } \\
\quad(\mathrm{kpc})\end{array}$ & $h_{0}^{\mathrm{sd}}$ & $C_{21}^{95 \%}$ & $C_{22}^{95 \%}$ & $h_{0}^{95 \%}$ & $\begin{array}{c}Q_{22}^{95 \%} \\
\left(\mathrm{~kg} \mathrm{~m}^{2}\right)\end{array}$ & $\varepsilon^{95 \%}$ & $h_{0}^{95 \%} / h_{0}^{\text {sd }}$ & $\mathcal{O}_{m=1,2}^{l=2}$ & $\mathcal{O}_{m=2}^{l=2}$ \\
\hline $\mathrm{J} 1748-30^{\mathrm{b}}$ & 103.3 & & 13.81 (b) & ‥ & $3.5 \times 10^{-26}$ & $6.6 \times 10^{-27}$ & $1.4 \times 10^{-26}$ & $3.3 \times 10^{32}$ & $4.3 \times 10^{-6}$ & $\cdots$ & -3.0 & -1.8 \\
\hline J1750-2536 & 28.8 & $8.1 \times 10^{-20}$ & 3.22 (b) & $3.8 \times 10^{-28}$ & $1.2 \times 10^{-25}$ & $1.1 \times 10^{-26}$ & $2.0 \times 10^{-26}$ & $1.4 \times 10^{33}$ & $1.8 \times 10^{-5}$ & 52 & -4.6 & -2.4 \\
\hline $\mathrm{J} 1751-2857^{\mathrm{a}}$ & 255.4 & $1.0 \times 10^{-20}$ & 1.09 (b) & $1.2 \times 10^{-27}$ & $1.5 \times 10^{-26}$ & $8.5 \times 10^{-27}$ & $1.8 \times 10^{-26}$ & $5.5 \times 10^{30}$ & $7.2 \times 10^{-8}$ & 15 & -3.8 & -2.0 \\
\hline J1753-1914 & 15.9 & $2.0 \times 10^{-18}$ & 2.91 (b) & $1.6 \times 10^{-27}$ & $1.9 \times 10^{-24}$ & $2.3 \times 10^{-26}$ & $4.7 \times 10^{-26}$ & $9.9 \times 10^{33}$ & $1.3 \times 10^{-4}$ & 30 & -4.5 & -2.7 \\
\hline J1753-2240 & 10.5 & $9.7 \times 10^{-19}$ & 3.23 (b) & $8.0 \times 10^{-28}$ & $2.2 \times 10^{-23}$ & $1.6 \times 10^{-25}$ & $3.2 \times 10^{-25}$ & $1.7 \times 10^{35}$ & $2.2 \times 10^{-3}$ & 410 & -4.0 & -2.2 \\
\hline $\mathrm{J} 1756-2251^{\mathrm{a}}$ & 35.1 & $1.0 \times 10^{-18}$ & $0.73(w)$ & $6.6 \times 10^{-27}$ & $5.7 \times 10^{-26}$ & $7.1 \times 10^{-27}$ & $1.5 \times 10^{-26}$ & $1.6 \times 10^{32}$ & $2.1 \times 10^{-6}$ & 2.3 & -4.8 & -2.3 \\
\hline J1757-27 & 56.5 & $2.1 \times 10^{-19}$ & 8.12 (b) & $3.4 \times 10^{-28}$ & $3.4 \times 10^{-26}$ & $7.2 \times 10^{-27}$ & $1.4 \times 10^{-26}$ & $6.3 \times 10^{32}$ & $8.2 \times 10^{-6}$ & 40 & -4.1 & -2.0 \\
\hline $\mathrm{J} 1801-1417^{\mathrm{a}}$ & 275.9 & $3.8 \times 10^{-21}$ & 1.10 (b) & $7.5 \times 10^{-28}$ & $2.0 \times 10^{-26}$ & $8.1 \times 10^{-27}$ & $1.8 \times 10^{-26}$ & $4.7 \times 10^{30}$ & $6.1 \times 10^{-8}$ & 24 & -3.7 & -1.9 \\
\hline $\mathrm{J} 1801-3210^{\mathrm{b}}$ & 134.2 & $\ldots$ & $6.12(b)$ & $\ldots$ & $1.3 \times 10^{-26}$ & $4.1 \times 10^{-27}$ & $9.0 \times 10^{-27}$ & $5.6 \times 10^{31}$ & $7.2 \times 10^{-7}$ & $\ldots$ & -4.1 & -2.1 \\
\hline J1802-2124 & 79.1 & $7.2 \times 10^{-20}(\mathrm{~g})$ & $0.64(\mathrm{~g})$ & $3.0 \times 10^{-27}$ & $2.5 \times 10^{-26}$ & $4.4 \times 10^{-27}$ & $9.4 \times 10^{-27}$ & $1.8 \times 10^{31}$ & $2.3 \times 10^{-7}$ & 3.1 & -4.0 & -2.1 \\
\hline $\mathrm{J} 1804-0735^{\mathrm{c}}$ & 43.3 & $1.8 \times 10^{-19}$ & $7.80(\mathrm{x})$ & $2.9 \times 10^{-28}$ & $4.4 \times 10^{-26}$ & $6.4 \times 10^{-27}$ & $1.3 \times 10^{-26}$ & $1.0 \times 10^{33}$ & $1.3 \times 10^{-5}$ & 45 & -4.7 & -2.3 \\
\hline $\mathrm{J} 1804-2717^{\mathrm{a}}$ & 107.0 & $3.5 \times 10^{-20}$ & $0.80(\mathrm{~b})$ & $1.9 \times 10^{-27}$ & $1.8 \times 10^{-26}$ & $4.7 \times 10^{-27}$ & $9.8 \times 10^{-27}$ & $1.2 \times 10^{31}$ & $1.6 \times 10^{-7}$ & 5 & -3.8 & -2.0 \\
\hline $\mathrm{J} 1807-2459 \mathrm{~A}^{\mathrm{c}}$ & 326.9 & $2.4 \times 10^{-20}$ & $2.79(\mathrm{y})$ & $8.1 \times 10^{-28}$ & $1.8 \times 10^{-26}$ & $2.1 \times 10^{-26}$ & $4.2 \times 10^{-26}$ & $2.0 \times 10^{31}$ & $2.6 \times 10^{-7}$ & 52 & -2.5 & -0.5 \\
\hline $\mathrm{J} 1810+1744$ & 601.4 & $4.5 \times 10^{-21}$ & $2.36(\mathrm{~b})$ & $5.6 \times 10^{-28}$ & $2.0 \times 10^{-26}$ & $1.6 \times 10^{-26}$ & $3.5 \times 10^{-26}$ & $4.2 \times 10^{30}$ & $5.4 \times 10^{-8}$ & 63 & -4.0 & -1.9 \\
\hline $\mathrm{J} 1810-2005^{\mathrm{a}}$ & 30.5 & $5.3 \times 10^{-20}$ & 3.51 (b) & $2.9 \times 10^{-28}$ & $2.0 \times 10^{-25}$ & $6.3 \times 10^{-27}$ & $1.6 \times 10^{-26}$ & $1.1 \times 10^{33}$ & $1.5 \times 10^{-5}$ & 56 & -3.9 & -2.6 \\
\hline $\mathrm{J} 1811-2405$ & 375.9 & $1.3 \times 10^{-20}(\mathrm{kk})$ & $1.83(\mathrm{~b})$ & $9.7 \times 10^{-28}$ & $2.0 \times 10^{-26}$ & $1.0 \times 10^{-26}$ & $2.1 \times 10^{-26}$ & $4.9 \times 10^{30}$ & $6.3 \times 10^{-8}$ & 21 & -3.9 & -2.1 \\
\hline $\mathrm{J} 1813-2621^{\mathrm{b}}$ & 225.7 & $\ldots$ & 3.01 (b) & $\ldots$ & $1.6 \times 10^{-26}$ & $5.1 \times 10^{-27}$ & $1.1 \times 10^{-26}$ & $1.2 \times 10^{31}$ & $1.5 \times 10^{-7}$ & $\cdots$ & -4.0 & -2.1 \\
\hline $\mathrm{J} 1816+4510^{\mathrm{a}}$ & 313.2 & $4.3 \times 10^{-20}$ & 4.36 (b) & $6.8 \times 10^{-28}$ & $1.9 \times 10^{-26}$ & $7.0 \times 10^{-27}$ & $1.4 \times 10^{-26}$ & $1.1 \times 10^{31}$ & $1.5 \times 10^{-7}$ & 21 & -3.9 & -2.1 \\
\hline $\mathrm{J} 1823-3021 \mathrm{~A}$ & 183.8 & $3.4 \times 10^{-18}$ & 8.40 (aa) & $2.4 \times 10^{-27}$ & $2.7 \times 10^{-26}$ & $9.7 \times 10^{-27}$ & $2.0 \times 10^{-26}$ & $9.3 \times 10^{31}$ & $1.2 \times 10^{-6}$ & 8.6 & -2.6 & -1.1 \\
\hline J1824-2452A & 327.4 & $1.6 \times 10^{-18}$ & $5.10(\mathrm{bb})$ & $3.6 \times 10^{-27}$ & $2.3 \times 10^{-26}$ & $1.0 \times 10^{-26}$ & $2.0 \times 10^{-26}$ & $1.7 \times 10^{31}$ & $2.3 \times 10^{-7}$ & 5.5 & -3.9 & -2.0 \\
\hline $\mathrm{J} 1825-0319$ & 219.6 & $6.8 \times 10^{-21}$ & 3.86 (b) & $2.6 \times 10^{-28}$ & $2.3 \times 10^{-26}$ & $7.9 \times 10^{-27}$ & $1.5 \times 10^{-26}$ & $2.2 \times 10^{31}$ & $2.9 \times 10^{-7}$ & 60 & -3.5 & -1.9 \\
\hline $\mathrm{J} 1827-0849$ & 445.9 & $1.1 \times 10^{-20}$ & $\ldots$ & $\ldots$ & $2.2 \times 10^{-26}$ & $9.6 \times 10^{-27}$ & $2.1 \times 10^{-26}$ & & $\ldots$ & $\ldots$ & -4.0 & -2.2 \\
\hline $\mathrm{J} 1832-0836^{\mathrm{b}}$ & 367.8 & $\ldots$ & 2.50 (a) & $\ldots$ & $2.2 \times 10^{-26}$ & $6.9 \times 10^{-27}$ & $1.4 \times 10^{-26}$ & $4.8 \times 10^{30}$ & $6.3 \times 10^{-8}$ & $\ldots$ & -4.1 & -2.3 \\
\hline J1840-0643 & 28.1 & $2.2 \times 10^{-16}$ & $5.01(\mathrm{~b})$ & $1.3 \times 10^{-26}$ & $9.1 \times 10^{-26}$ & $1.8 \times 10^{-26}$ & $3.5 \times 10^{-26}$ & $4.0 \times 10^{33}$ & $5.2 \times 10^{-5}$ & 2.8 & -3.5 & -1.2 \\
\hline $\mathrm{J} 1841+0130$ & 33.6 & $8.2 \times 10^{-18}$ & 4.23 (b) & $3.2 \times 10^{-27}$ & $7.3 \times 10^{-26}$ & $6.4 \times 10^{-27}$ & $1.4 \times 10^{-26}$ & $9.6 \times 10^{32}$ & $1.2 \times 10^{-5}$ & 4.4 & -4.6 & -2.4 \\
\hline $\mathrm{J} 1843-1113$ & 541.8 & $9.4 \times 10^{-21}(\mathrm{~g})$ & $1.48(\mathrm{~s})$ & $1.2 \times 10^{-27}$ & $2.2 \times 10^{-26}$ & $2.2 \times 10^{-26}$ & $4.6 \times 10^{-26}$ & $4.2 \times 10^{30}$ & $5.5 \times 10^{-8}$ & 37 & -3.6 & -1.6 \\
\hline $\mathrm{J} 1844+0115$ & 238.9 & $1.1 \times 10^{-20}$ & 4.36 (b) & $3.0 \times 10^{-28}$ & $1.4 \times 10^{-26}$ & $6.2 \times 10^{-27}$ & $1.3 \times 10^{-26}$ & $1.9 \times 10^{31}$ & $2.4 \times 10^{-7}$ & 45 & -4.0 & -2.1 \\
\hline $\mathrm{J} 1850+0124$ & 280.9 & $1.1 \times 10^{-20}$ & 3.39 (b) & $4.2 \times 10^{-28}$ & $1.8 \times 10^{-26}$ & $7.5 \times 10^{-27}$ & $1.6 \times 10^{-26}$ & $1.3 \times 10^{31}$ & $1.6 \times 10^{-7}$ & 39 & -3.8 & -2.1 \\
\hline $\mathrm{J} 1853+1303^{\mathrm{a}}$ & 244.4 & $8.7 \times 10^{-21}$ & 1.32 (b) & $8.9 \times 10^{-28}$ & $2.5 \times 10^{-26}$ & $9.8 \times 10^{-27}$ & $2.2 \times 10^{-26}$ & $8.9 \times 10^{30}$ & $1.1 \times 10^{-7}$ & 25 & -3.4 & -1.8 \\
\hline J1855-1436 & 278.2 & $1.1 \times 10^{-20}$ & 5.15 (b) & $2.7 \times 10^{-28}$ & $2.3 \times 10^{-26}$ & $1.0 \times 10^{-26}$ & $2.0 \times 10^{-26}$ & $2.5 \times 10^{31}$ & $3.2 \times 10^{-7}$ & 74 & -3.4 & -1.8 \\
\hline $\mathrm{J} 1857+0943$ & 186.5 & $1.7 \times 10^{-20}(\mathrm{~g})$ & $1.10(\mathrm{~g})$ & $1.3 \times 10^{-27}$ & $1.3 \times 10^{-26}$ & $4.5 \times 10^{-27}$ & $1.0 \times 10^{-26}$ & $5.8 \times 10^{30}$ & $7.6 \times 10^{-8}$ & 7.7 & -4.2 & -2.2 \\
\hline J1858-2216 & 419.5 & $3.9 \times 10^{-21}$ & 0.92 (b) & $1.1 \times 10^{-27}$ & $2.4 \times 10^{-26}$ & $8.7 \times 10^{-27}$ & $1.9 \times 10^{-26}$ & $1.8 \times 10^{30}$ & $2.4 \times 10^{-8}$ & 17 & -3.8 & -2.1 \\
\hline $\mathrm{J} 1900+0308$ & 203.7 & $5.9 \times 10^{-21}$ & 4.80 (b) & $1.8 \times 10^{-28}$ & $2.1 \times 10^{-26}$ & $5.0 \times 10^{-27}$ & $1.1 \times 10^{-26}$ & $2.3 \times 10^{31}$ & $2.9 \times 10^{-7}$ & 58 & -3.8 & -2.2 \\
\hline $\mathrm{J} 1902-5105^{\mathrm{a}}$ & 573.9 & $8.7 \times 10^{-21}$ & 1.65 (b) & $1.1 \times 10^{-27}$ & $2.1 \times 10^{-26}$ & $1.4 \times 10^{-26}$ & $2.9 \times 10^{-26}$ & $2.7 \times 10^{30}$ & $3.5 \times 10^{-8}$ & 27 & -4.1 & -2.1 \\
\hline $\mathrm{J} 1903+0327^{\mathrm{a}}$ & 465.1 & $2.0 \times 10^{-20}$ & $6.11(\mathrm{~b})$ & $4.0 \times 10^{-28}$ & $2.5 \times 10^{-26}$ & $9.7 \times 10^{-27}$ & $2.1 \times 10^{-26}$ & $1.1 \times 10^{31}$ & $1.4 \times 10^{-7}$ & 52 & -3.9 & -2.1 \\
\hline $\mathrm{J} 1903-7051^{\mathrm{a}}$ & 277.9 & $7.7 \times 10^{-21}$ & 0.93 (b) & $1.3 \times 10^{-27}$ & $2.0 \times 10^{-26}$ & $7.2 \times 10^{-27}$ & $1.6 \times 10^{-26}$ & $3.5 \times 10^{30}$ & $4.5 \times 10^{-8}$ & 13 & -3.7 & -2.0 \\
\hline J1904+0412 & 14.1 & $1.1 \times 10^{-19}$ & 4.58 (b) & $2.2 \times 10^{-28}$ & $3.6 \times 10^{-24}$ & $4.3 \times 10^{-26}$ & $7.9 \times 10^{-26}$ & $3.3 \times 10^{34}$ & $4.3 \times 10^{-4}$ & 360 & -4.3 & -2.3 \\
\hline J1904+0451 & 164.1 & $5.7 \times 10^{-21}$ & 4.40 (b) & $1.8 \times 10^{-28}$ & $1.5 \times 10^{-26}$ & $4.9 \times 10^{-27}$ & $1.1 \times 10^{-26}$ & $3.2 \times 10^{31}$ & $4.1 \times 10^{-7}$ & 60 & -4.2 & -2.3 \\
\hline $\mathrm{J} 1905+0400^{\mathrm{a}}$ & 264.2 & $4.2 \times 10^{-21}$ & $1.06(b)$ & $8.0 \times 10^{-28}$ & $1.4 \times 10^{-26}$ & $8.3 \times 10^{-27}$ & $1.8 \times 10^{-26}$ & $4.9 \times 10^{30}$ & $6.4 \times 10^{-8}$ & 22 & -3.9 & -1.9 \\
\hline J1908+2105 & 390.0 & $1.4 \times 10^{-20}$ & 2.58 (b) & $7.3 \times 10^{-28}$ & $2.5 \times 10^{-26}$ & $1.3 \times 10^{-26}$ & $2.5 \times 10^{-26}$ & $7.7 \times 10^{30}$ & $9.9 \times 10^{-8}$ & 34 & -3.4 & -1.9 \\
\hline J1909-3744 & 339.3 & $2.7 \times 10^{-21}(\mathrm{~g})$ & $1.15(\mathrm{~g})$ & $6.7 \times 10^{-28}$ & $2.5 \times 10^{-26}$ & $1.6 \times 10^{-26}$ & $3.2 \times 10^{-26}$ & $5.8 \times 10^{30}$ & $7.5 \times 10^{-8}$ & 47 & -3.1 & -1.3 \\
\hline $\mathrm{J} 1910+1256$ & 200.7 & $9.3 \times 10^{-21}(\mathrm{~g})$ & $1.16(\mathrm{~s})$ & $9.5 \times 10^{-28}$ & $2.5 \times 10^{-26}$ & $5.5 \times 10^{-27}$ & $1.2 \times 10^{-26}$ & $6.4 \times 10^{30}$ & $8.3 \times 10^{-8}$ & 13 & -3.5 & -2.1 \\
\hline$J 1910-5959 A^{c}$ & 306.2 & $2.6 \times 10^{-20}$ & 4.50 (ee) & $5.0 \times 10^{-28}$ & $1.9 \times 10^{-26}$ & $6.3 \times 10^{-27}$ & $1.4 \times 10^{-26}$ & $1.2 \times 10^{31}$ & $1.6 \times 10^{-7}$ & 27 & -4.1 & -2.2 \\
\hline$J 1910-5959 C^{c}$ & 189.5 & $4.2 \times 10^{-20}$ & $4.50(\mathrm{ee})$ & $5.0 \times 10^{-28}$ & $1.6 \times 10^{-26}$ & $4.9 \times 10^{-27}$ & $1.1 \times 10^{-26}$ & $2.4 \times 10^{31}$ & $3.1 \times 10^{-7}$ & 21 & -3.9 & -2.2 \\
\hline $\mathrm{J} 1910-5959 \mathrm{D}^{\mathrm{c}}$ & 110.7 & $7.2 \times 10^{-20}$ & $4.50(\mathrm{ee})$ & $5.0 \times 10^{-28}$ & $2.2 \times 10^{-26}$ & $5.3 \times 10^{-27}$ & $1.2 \times 10^{-26}$ & $7.7 \times 10^{31}$ & $1.0 \times 10^{-6}$ & 23 & -3.4 & -1.9 \\
\hline $\mathrm{J} 1911+1347^{\mathrm{a}}$ & 216.2 & $1.7 \times 10^{-20}$ & $1.36(\mathrm{~b})$ & $1.1 \times 10^{-27}$ & $1.5 \times 10^{-26}$ & $5.2 \times 10^{-27}$ & $1.2 \times 10^{-26}$ & $6.1 \times 10^{30}$ & $7.9 \times 10^{-8}$ & 10 & -4.0 & -2.1 \\
\hline $\mathrm{J} 1911-1114^{\mathrm{a}}$ & 275.8 & $1.1 \times 10^{-20}$ & 1.07 (b) & $1.3 \times 10^{-27}$ & $1.7 \times 10^{-26}$ & $1.1 \times 10^{-26}$ & $2.2 \times 10^{-26}$ & $5.6 \times 10^{30}$ & $7.2 \times 10^{-8}$ & 16 & -3.5 & -1.6 \\
\hline
\end{tabular}


Table 2

(Continued)

\begin{tabular}{|c|c|c|c|c|c|c|c|c|c|c|c|c|}
\hline $\begin{array}{l}\text { Pulsar Name } \\
\text { (J2000) }\end{array}$ & $\begin{array}{l}f_{\text {rot }} \\
(\mathrm{Hz})\end{array}$ & $\underset{\left(\mathrm{P} \mathrm{s}^{-1}\right)}{\dot{P}_{\text {ot }}}$ & $\begin{array}{l}\text { Distance } \\
(\mathrm{kpc})\end{array}$ & $h_{0}^{\text {sd }}$ & $C_{21}^{95 \%}$ & $C_{22}^{95 \%}$ & $h_{0}^{95 \%}$ & $\begin{array}{c}Q_{22}^{95 \%} \\
\left(\mathrm{~kg} \mathrm{~m}^{2}\right)\end{array}$ & $\varepsilon^{95 \%}$ & $h_{0}^{95 \%} / h_{0}^{\text {sd }}$ & $\mathcal{O}_{m=1,2}^{l=2}$ & $\mathcal{O}_{m=2}^{l=2}$ \\
\hline J2256-1024 & 435.8 & $1.1 \times 10^{-20}$ & 1.33 (b) & $1.3 \times 10^{-27}$ & $2.6 \times 10^{-26}$ & $1.2 \times 10^{-26}$ & $2.3 \times 10^{-26}$ & $2.9 \times 10^{30}$ & $3.8 \times 10^{-8}$ & 17 & -3.7 & -2.1 \\
\hline J2310-0555 & 382.8 & $5.0 \times 10^{-21}$ & 1.55 (b) & $7.2 \times 10^{-28}$ & $1.9 \times 10^{-26}$ & $9.7 \times 10^{-27}$ & $2.0 \times 10^{-26}$ & $3.9 \times 10^{30}$ & $5.0 \times 10^{-8}$ & 28 & -4.0 & -2.1 \\
\hline $\mathbf{J} 2317+1439$ & 290.3 & $3.5 \times 10^{-21}(\mathrm{~g})$ & $1.01(\mathrm{~g})$ & $8.0 \times 10^{-28}$ & $1.5 \times 10^{-26}$ & $1.2 \times 10^{-26}$ & $2.6 \times 10^{-26}$ & $5.6 \times 10^{30}$ & $7.2 \times 10^{-8}$ & 32 & -3.6 & -1.6 \\
\hline J2322+2057 & 208.0 & $4.4 \times 10^{-22}$ (ii) & 0.23 (ii) & $1.1 \times 10^{-27}$ & $2.1 \times 10^{-26}$ & $6.2 \times 10^{-27}$ & $1.3 \times 10^{-26}$ & $1.3 \times 10^{30}$ & $1.6 \times 10^{-8}$ & 12 & -3.7 & -2.0 \\
\hline $\mathrm{J} 2339-0533^{\mathrm{a}}$ & 346.7 & $6.9 \times 10^{-21}$ & $1.10(\mathrm{jj})$ & $1.1 \times 10^{-27}$ & $2.2 \times 10^{-26}$ & $8.1 \times 10^{-27}$ & $1.8 \times 10^{-26}$ & $2.9 \times 10^{30}$ & $3.8 \times 10^{-8}$ & 15 & -4.9 & -2.4 \\
\hline
\end{tabular}

Notes. The information in Table 2 is available in the machine readable version of Table 1.

${ }^{a}$ The observed $\dot{P}$ has been corrected to account for the relative motion between the pulsar and observer.

b The corrected pulsar $\dot{P}$ value is negative, so no value is given and no spin-down limit has been calculated.

${ }^{\mathrm{c}}$ This is a globular cluster pulsar for which a proxy period derivative has been derived assuming a characteristic age of $10^{9} \mathrm{yr}$ and a braking index of $n=5$.

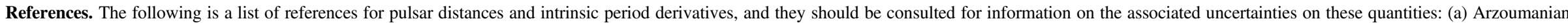

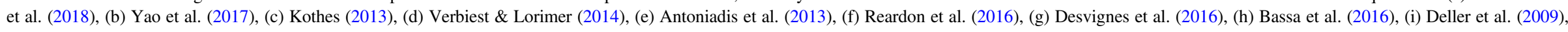

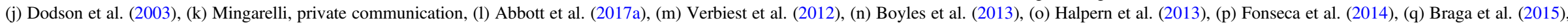

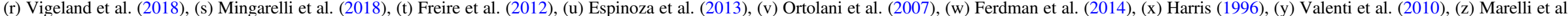

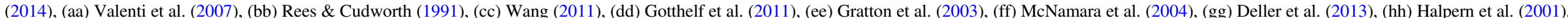
(ii) Spiewak et al. (2018), (jj) Romani \& Shaw (2011), (kk) Ng et al. (2014). 


\section{Results}

For each pulsar the results presented here are from analyses coherently combining the data from both the LIGO detectors. As described below, we see no strong evidence for a gravitational-wave signal from any pulsar, so we therefore cast our results in terms of upper limits on the gravitationalwave amplitude. These limits are subject to the uncertainties from the detector calibration as described in Section 2.1, as well as statistical uncertainties that are dependent on the particular analysis method used. For the Bayesian analysis, statistical uncertainties on the $95 \%$ credible upper limits are on the order of $1 \%$ (see Figure 12 of Pitkin et al. 2017). For the $5 n$-vector method the statistical uncertainty on the upper limits is of the order of $1 \%-5 \%$, depending on the pulsar.

For all pulsars, we present the results of our analyses in terms of several quantities. For the searches including data at both once and twice the rotation frequency and searching for a signal from both the $l=2, m=1,2$ modes we present the inferred limits on the $C_{21}$ and $C_{22}$ amplitude parameters given in Equations (1) and (2). For the searches looking only for emission from the $l=m=2$ mode we present limits on the signal's gravitational-wave strain $h_{0}$. For the Bayesian search these limits are $95 \%$ credible upper bounds derived from the posterior probability distributions. For the $5 n$-vector pipeline the upper limits are obtained with a hybrid frequentist/ Bayesian approach, described in Appendix D, consisting in evaluating the posterior probability distribution of the signal amplitude $H_{0}$, conditioned to the measured value of a detection statistic, and converting it to a $95 \%$ credible upper limit on $h_{0}$ or $C_{21}$ (see Section 1.3, Appendix E, and Aasi et al. 2014, for more details.) Upper limits have been computed assuming both flat and, when information from electromagnetic observation is available, restricted priors on the polarization parameters, as detailed in Section 2.2.4 and Appendix B.

For the purely $l=m=2$ mode search, we are able to convert these limits into equivalent limits on several derived quantities. In cases where we have an estimate for the pulsar distance (see Section 2.2 and Tables 1 and 2) $h_{0}$ can be converted directly into a limit on the $Q_{22}$ mass quadrupole (see Equation (5)). Under the assumption of a fiducial principal moment of inertia of $I_{z z}^{\mathrm{fid}}=10^{38} \mathrm{~kg} \mathrm{~m}^{2}$ this can also place a limit on the fiducial ellipticity $\varepsilon$. When we also have a reliable estimate of the intrinsic period derivative, the spin-down limit $h_{0}^{\text {sd }}$ can be calculated (see Equation (9)) and the ratio of the observed limits on $h_{0}$ to this value, $h_{0}^{95 \%} / h_{0}^{\text {sd }}$, is shown (the square of this value gives the ratio of the limit on the gravitational-wave luminosity to the spin-down luminosity of the pulsar).

For the Bayesian method, an odds value giving a ratio of probabilities is also calculated (the base-10 logarithm of which we denote as $\mathcal{O}$, which is equivalent to $\log _{10} \mathcal{O}_{\mathrm{S} / \mathrm{I}}$ from Abbott et al. 2017a), where the numerator is the probability of the data being consistent with a coherent signal model in both detectors and the denominator is the probability of an incoherent signal present in both detectors or Gaussian noise in one detector and a signal in the other or Gaussian noise being present in both detectors (see Appendix A.3 in Abbott et al. 2017a or Section 2.6 of Pitkin et al. 2017 for more details). These odds can be used to assess when the coherent signal model is favored by the data. The values of $\mathcal{O}$ for each pulsar are shown in Tables 1 (where it is the value given in the "Statistic" column for the Bayesian search) and 2, but in all cases the values are negative, indicating no pulsars for which the coherent signal model is favored. Also, examination of the posterior probability distributions for the amplitude parameters shows that none are significantly disjoint from the probability of the amplitude being zero.

In the 5n-vector search the significance of each analysis is expressed through a $p$-value, which is a measure of how compatible the data are with pure noise. It is obtained by empirically computing the noise-only distribution of the detection statistic, over an off-source region, and comparing it to the value of the detection statistic found in the actual analysis. Conventionally, a threshold of $p<0.01$ on the $p$-value is used to identify potentially interesting candidates: pulsars for which the analysis provides a $p$-value smaller than the threshold would deserve a deeper study (see also Aasi et al. 2014; Abbott et al. 2017a). The computed $p$-values are reported in Table 1. For all the analyzed pulsars they are well above $p=0.01$, suggesting that the data are fully compatible with noise.

For the $\mathcal{F}$ - $/ \mathcal{G}$-statistic method false-alarm probabilities of obtaining the observed statistic values are calculated. They are derived assuming that for the $\mathcal{F}$-statistic the $2 \mathcal{F}$ value has a $\chi^{2}$ distribution with 4 degrees of freedom (Jaranowski et al. 1998) and for the $\mathcal{G}$-statistic the $2 \mathcal{G}$ value has a $\chi^{2}$ distribution with 2 degrees of freedom (Jaranowski \& Królak 2010). The falsealarm probabilities reported in Table 1 are all close to unity and show no strong indication that the statistics deviate from their expected distributions.

The results for the 34 high-value targets are shown in Table 1, and the results for all the other pulsars are shown in Table 2. The $95 \%$ credible upper limits on $C_{21}$ and $C_{22}$ for all 222 pulsars from the Bayesian analysis are shown as a function of the gravitational-wave emission frequency in Figure 1. Also shown are estimates of the expected sensitivity of the search given representative noise amplitude spectral densities from the $\mathrm{O} 1$ and $\mathrm{O} 2$ observing runs (see Appendix $\mathrm{C}$ for descriptions of how these were produced). The $95 \%$ credible upper limits on $h_{0}$ for all 222 pulsars from the search purely for emission from the $l=m=2$ mode are shown in Figure 2. Figure 2 also shows spin-down limits on the emission as triangles, and in the cases where our observed upper limits are below these the result is highlighted with a circular marker and is linked to its associated spin-down limit with a vertical line.

Figure 3 shows a histogram of the spin-down ratio $h_{0}^{95 \%} / h_{0}^{\text {sd }}$ from the Bayesian analysis for the $l=m=2$ mode search, for pulsars where it was possible to calculate a spin-down limit. This shows 20 pulsars for which $h_{0}^{95 \%}<h_{0}^{\text {sd }}$ and 53 for which the results are between 1 and 10 times greater than $h_{0}^{\text {sd }}$. If we just look at MSPs, then 41 are within a factor of 10 of the spindown limit. ${ }^{202}$ The spin-down limits and the $Q_{22}$ and $\varepsilon$ values assume a particular distance, intrinsic period derivative, and fiducial moment of inertia of $10^{38} \mathrm{~kg} \mathrm{~m}^{2}$, but there can be considerable uncertainties on these values. For example, distances calculated using the Galactic electron density model of Yao et al. (2017) have a $1 \sigma$ relative error of $\sim 40 \%$, with some parts of the sky having several $100 \%$ relative errors. The true moment of inertia depends on the pulsar's mass and equation of state and could be within a range of roughly

\footnotetext{
202 Based on our sample of pulsars with rotation frequencies greater than $10 \mathrm{~Hz}$, there is a clear distinction between the MSP and young (or normal) population based on a cut in $\dot{P}$ of $10^{-17} \mathrm{~s} \mathrm{~s}^{-1}$, i.e., we assume that any pulsar with a $\dot{P}$ smaller than this is an MSP.
} 


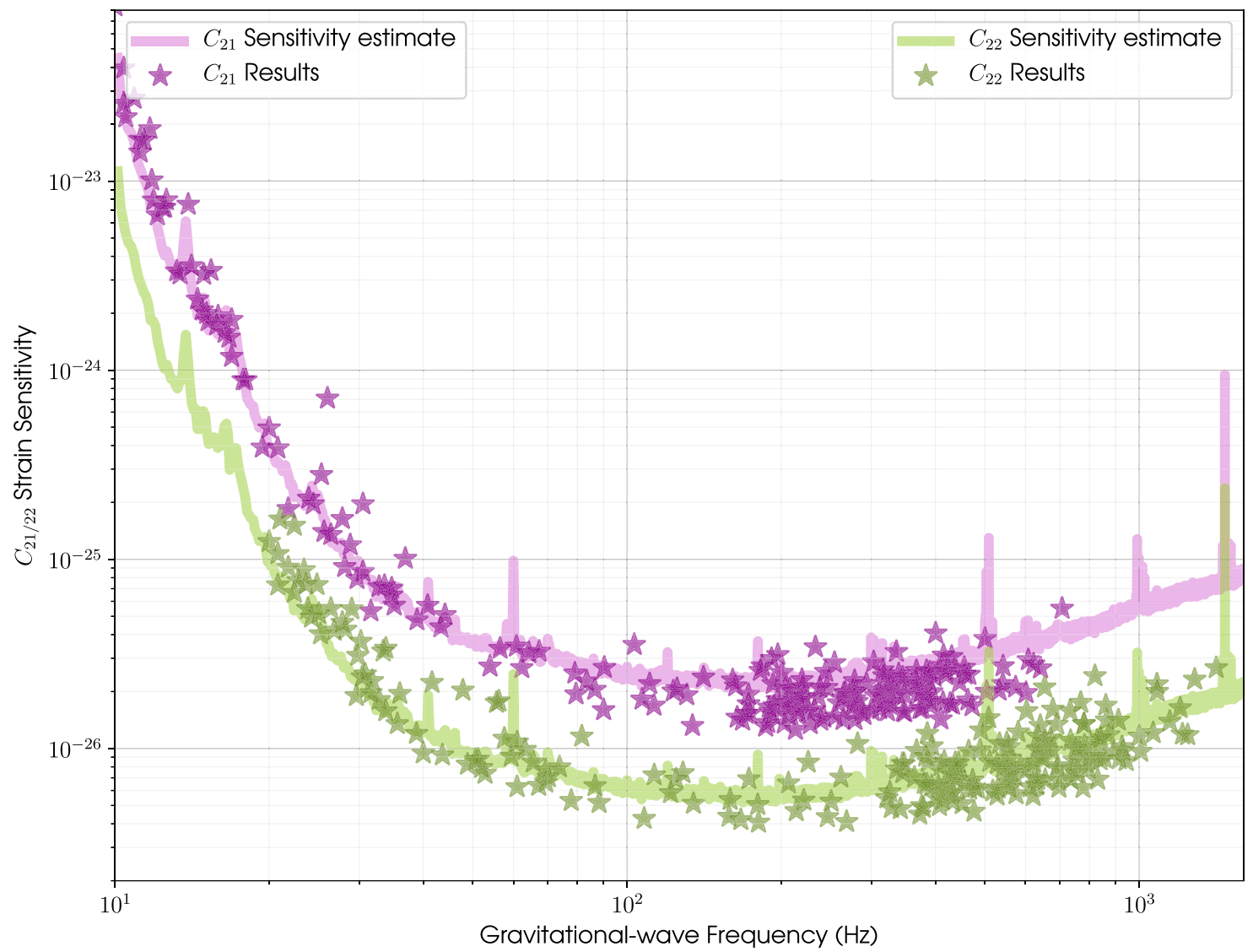

Figure 1. Upper limits on $C_{21}$ and $C_{22}$ for 222 pulsars. The stars show the observed $95 \%$ credible upper limits on observed amplitudes for each pulsar. The solid lines show an estimate of the expected sensitivity of the searches.

$(1-3) \times 10^{38} \mathrm{~kg} \mathrm{~m}^{2}$ (see, e.g., Figures 4 and 7 of Worley et al. 2008 and Figures 6 and 7 of Bejger 2013). We do not incorporate these uncertainties into the results we present here, but they should be kept in mind when interpreting the limits. ${ }^{203}$ In the case of pulsar distances the references provided in Tables 1 and 2 should be consulted to provide an estimate of the associated uncertainty. These uncertainties dominate the few percent uncertainties arising from the calibration of the gravitational-wave detectors described in Section 2.1.

The $h_{0}^{95 \%}$ results from the Bayesian analysis, recast as limits on $Q_{22}$ and the fiducial ellipticity and assuming the distances given in Tables 1 and 2, are shown in Figure 4. The much lower limits on $\varepsilon$ inferred for the MSPs easily follow from the frequency scaling seen in Equation (6).

\subsection{Results Highlights}

For decades, two of the most intriguing targets in searches for gravitational waves from pulsars have been the Crab and Vela pulsars (J0534+2200 and J0835-4510, respectively), due to their large spin-down luminosities. For these two pulsars, assuming emission from the $l=m=2$ mode and with the phase precisely locked to the observed rotational phase, the limits observed using the initial LIGO and Virgo detectors in Abbott et al. (2008) and Abadie et al. (2011), respectively,

\footnotetext{
${ }^{203}$ From Equations (4), (5), and (9) it can be seen that fractional uncertainties on distance will scale directly into the uncertainties on $\varepsilon, Q_{22}$, and $h_{0}^{\text {sd }}$. Increasing the value of $I_{z z}^{\text {fid }}$ will proportionally decrease the inferred $\varepsilon$ value and increase the inferred spin-down limit by a factor given by the square root of the fractional increase compared to the canonical moment of inertia.
}

were lower than the equivalent spin-down limits. Using data from the $\mathrm{O} 1$ run, the observed limits were also below the spindown limit for these two pulsars in searches where the strict phase locking of the observed rotational phase and gravitational-wave phase was relaxed (Abbott et al. 2017b). ${ }^{204}$

For the Crab pulsar, this analysis finds an observed $95 \%$ limit of $h_{0}^{95 \%}=1.9 \times 10^{-26}$ for the Bayesian analysis (with consistent values of $2.2 \times 10^{-26}$ and $2.9 \times 10^{-26}$ for the $\mathcal{F}$-statistic and $5 n$-vector analyses, respectively). This is 0.013 times the spin-down ratio, or, equivalently, it means that less than $0.017 \%$ of the available spin-down luminosity is emitted via gravitational waves (see Equation (7)). These limits are also well below less naive spin-down limits that can be calculated by taking into account the power radiated electromagnetically or through particle acceleration (Ostriker \& Gunn 1969; Palomba 2000). As shown in Table 1, slightly tighter constraints are possible if one assumes that the orientation of the pulsar matches that derived from the observed orientation of its pulsar wind nebula (see Section 2.2.4). The above $h_{0}$ upper limit corresponds to limits on $Q_{22}$ of $7.7 \times 10^{32} \mathrm{~kg} \mathrm{~m}^{2}$ and an equivalent fiducial ellipticity of $1.0 \times 10^{-5}$. This mass quadrupole is almost in the range of maximum allowable quadrupoles for standard neutron star equations of state (see discussion in Section 1.2 and Johnson-McDaniel \& Owen 2013).

\footnotetext{
${ }^{204}$ In the similar narrowband searches for the Crab pulsar in Abbott et al (2008) and Aasi et al. (2015b) the limits were also below the spin-down limit, under the assumption that the orientation was restricted to that derived from the pulsar wind nebula (see Section 2.2.4).
} 


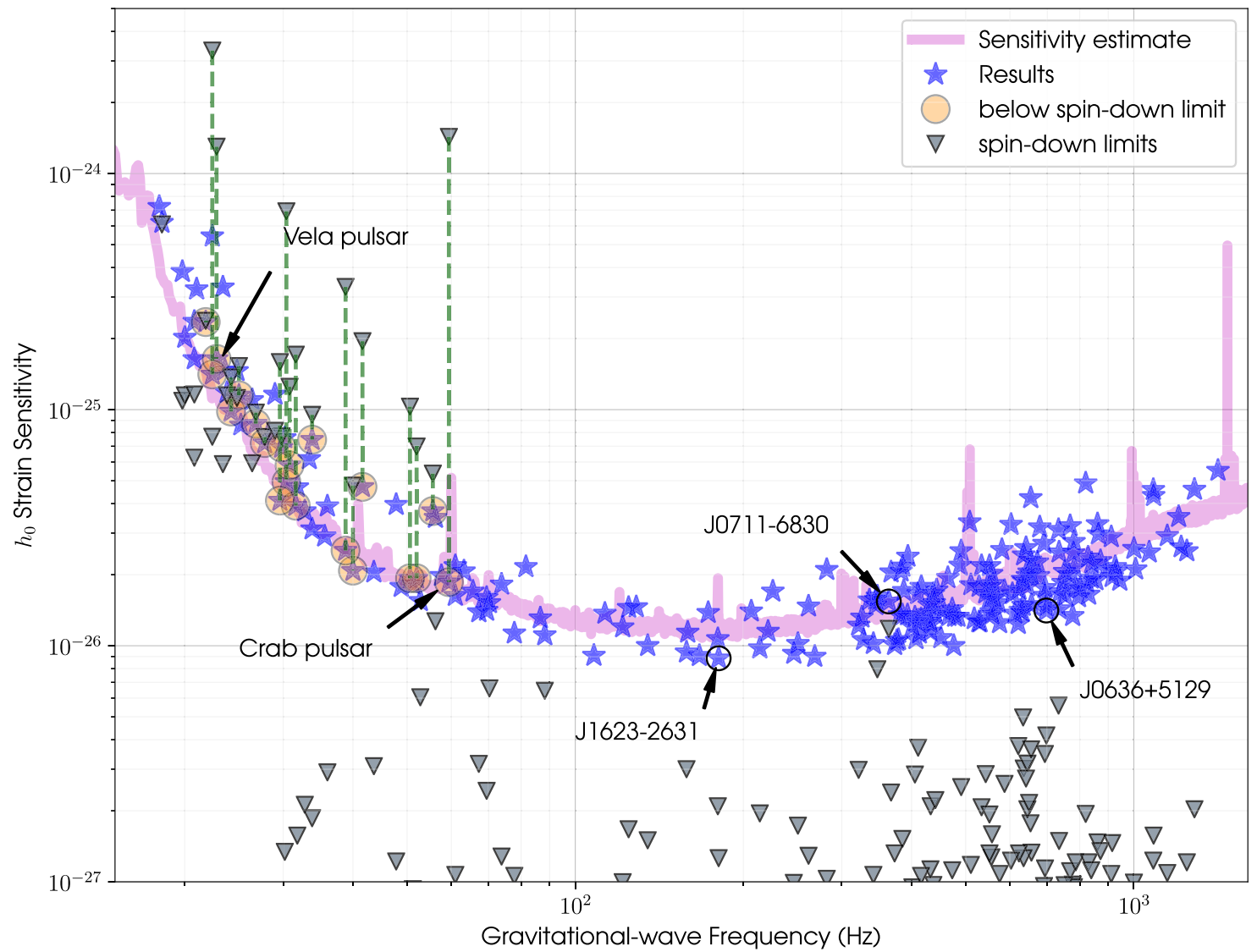

Figure 2. Upper limits on $h_{0}$ for 222 pulsars. The stars show the observed $95 \%$ credible upper limits on observed amplitude for each pulsar. The solid line shows an estimate of the expected sensitivity of the search. Triangles show the limits on gravitational-wave amplitude derived from each pulsar's observed spin-down.

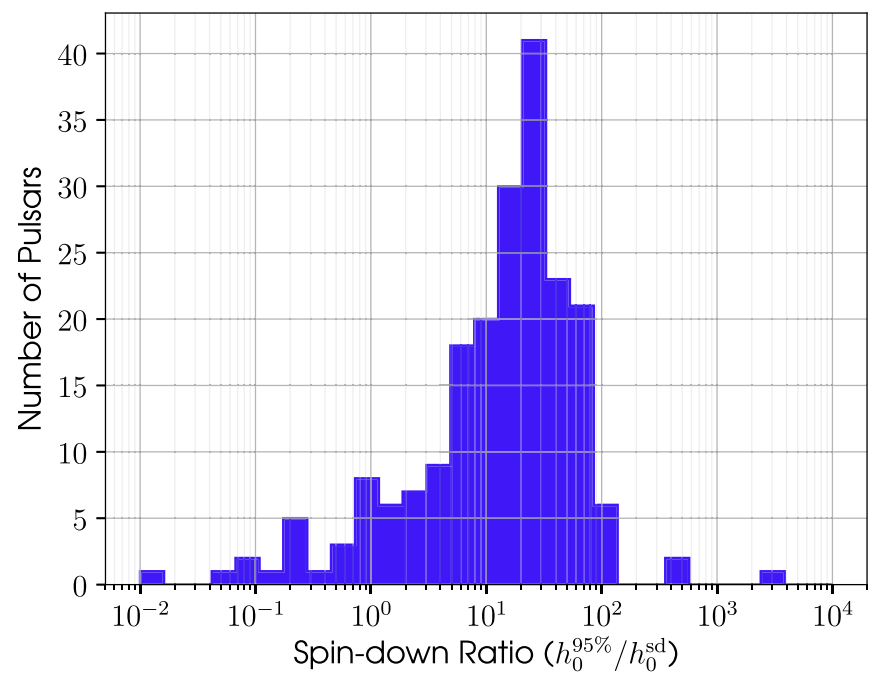

Figure 3. Histogram of ratios of upper limits on $h_{0}$ compared to the spin-down limit.

Similarly, for the Vela pulsar, this analysis finds an observed 95\% limit of $h_{0}^{95 \%}=1.4 \times 10^{-25}$ for the Bayesian analysis (with broadly consistent values of $2.6 \times 10^{-25}$ and $2.3 \times$ $10^{-25}$ for the $\mathcal{F}$-statistic and 5 -vector analyses, respectively). This is 0.042 times the spin-down ratio, or, equivalently, it means that less than $0.18 \%$ of the available spin-down luminosity is emitted via gravitational waves. The above $h_{0}$ upper limit corresponds to limits on $Q_{22}$ of $5.9 \times 10^{33} \mathrm{~kg} \mathrm{~m}^{2}$ and an equivalent fiducial ellipticity of $7.6 \times 10^{-5}$.
Of all the pulsars in the analysis, the one with the smallest upper limit on $h_{0}$ is PSR J1623-2631 (with a rotational frequency of $90.3 \mathrm{~Hz}$ and distance of $1.8 \mathrm{kpc}$ ), with $h_{0}^{95 \%}=$ $8.9 \times 10^{-27}$. The pulsar with the smallest limit on the $Q_{22}$ mass quadrupole is PSR J0636 +5129 (with a rotational frequency of $348.6 \mathrm{~Hz}$ and distance of $0.21 \mathrm{kpc}$ ), with $Q_{22}^{95 \%}$ of $4.5 \times 10^{29}$ and an equivalent fiducial ellipticity limit of $5.8 \times 10^{-9}$. These limits are only a factor of 3.4 above the pulsar's spin-down limit. Of the MSPs in our search (which, as above, we take as any pulsar with $\dot{P}<10^{-17} \mathrm{~s} \mathrm{~s}^{-1}$ ), the one for which our limit is closest to the spin-down limit is J0711 -6830 (with a rotational frequency of $182.1 \mathrm{~Hz}$ and a distance of $0.11 \mathrm{kpc})$. It is within a factor of 1.3 of the spin-down limit, with an observed upper limit of $h_{0}^{95 \%}=1.5 \times 10^{-26}$ and derived limits on $Q_{22}$ and ellipticity of $9.3 \times 10^{29} \mathrm{~kg} \mathrm{~m}^{2}$ and $1.2 \times 10^{-8}$, respectively. ${ }^{205}$ The upper bound on possible neutron star moments of inertia is roughly $3 \times 10^{38} \mathrm{~kg} \mathrm{~m}^{2}$, for which the fiducial spin-down limit could be increased by a factor of $\sqrt{3} \approx 1.7$, which would be greater than our upper limit.

\footnotetext{
$\overline{205}$ It is interesting to note that in Abbott et al. (2017a) PSR J0437-4715 was the MSP with an observed upper limit closest to its spin-down limit, being only a factor of 1.4 above that value, while J0711-6830 had a limit that was a factor of $\sim 20$ above its spin-down limit. For J0437-4715, despite now having an improved upper limit on the gravitational-wave amplitude, the correction of the observed period derivative to the intrinsic period derivative has lowered the spin-down limit by roughly a factor of two. For J0711-6830 the distance estimated using the YMW16 Galactic electron density model (Yao et al. 2017) is about a factor of 9 closer than that estimated with the previously used NE2001 model (Cordes \& Lazio 2002).
} 


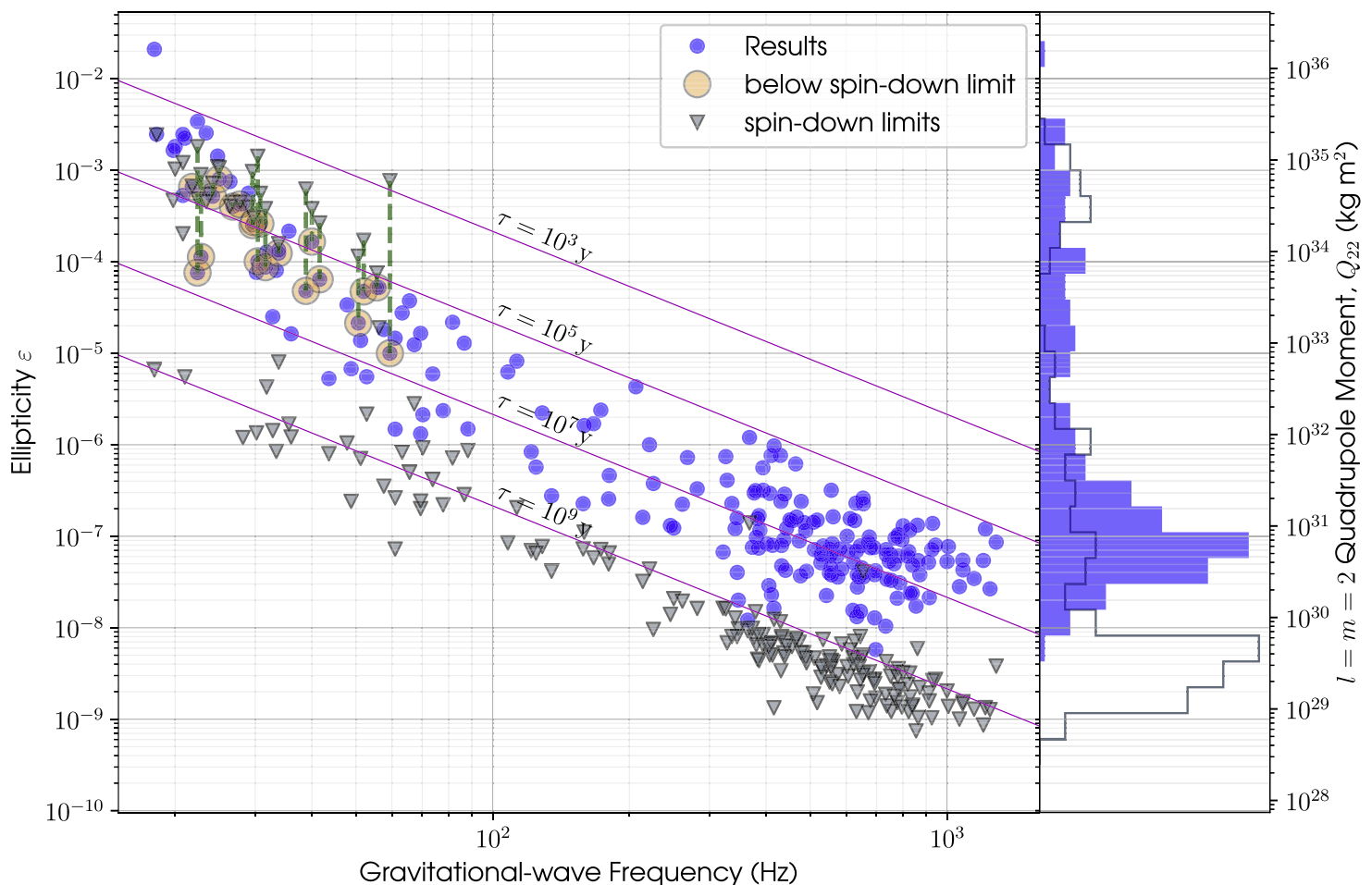

Figure 4. Upper limits on mass quadrupole $Q_{22}$ and fiducial ellipticity $\varepsilon$ for 222 pulsars. The filled circles show the limits as derived from the observed upper limits on the gravitational-wave amplitude $h_{0}$ assuming the canonical moment of inertia and distances given in Tables 1 and 2. Triangles show the limits derived from each pulsar's observed spin-down. The diagonal lines show contours of equal characteristic age $\tau$ assuming that braking is entirely through gravitational-wave emission. The distributions of these limits are also show in histogram form to the right of the figure, with the filled and unfilled histograms showing our observed limits and the spin-down limits, respectively.

Similarly to Abbott et al. (2017a), our most stringent limits on ellipticity for MSPs still imply limits on the internal toroidal magnetic field strength of $\lesssim 10^{9} \mathrm{~T}$ (or $10^{13} \mathrm{G}$ ) (applying Equation (2.4) of Cutler 2002, and assuming a superconducting core). The method in Mastrano \& Melatos (2012) could also be applied to these results to constrain the ratio of the poloidal magnetic field energy to the total field energy.

For the searches that include the $l=2, m=1$ mode, the smallest upper limit on the $C_{21}$ amplitude is for PSR J1744 -7619 (with a rotational frequency of $213.3 \mathrm{~Hz}$ ), at $C_{21}^{95 \%}=$ $1.3 \times 10^{-26}$. As $C_{21}$ and $C_{22}$ are not very strongly correlated, the upper limits on $C_{22}$ are generally consistent with $C_{22}^{95 \%} \approx h_{0}^{95 \%} / 2$.

\section{Discussion}

In this paper we have used data from the first two observation runs of Advanced LIGO (O1 and O2) to update the upper limits on the gravitational-wave amplitude $h_{0}$ for emission from the $l=m=2$ mass quadrupole for 167 pulsars. This compares to 271 results presented previously in Aasi et al. (2014) (using data from the initial runs of the LIGO [Abbott et al. 2009] and Virgo [Accadia et al. 2012] detectors, S1-6 and VSR1-4) and Abbott et al. (2017a) (using data from the first observing run, O1, of the advanced LIGO detectors; Aasi et al. 2015a; Abbott et al. 2016). New upper limits on $h_{0}$ have been set for a further 55 pulsars. Other than the results in Pitkin et al. (2015), we have also presented the first comprehensive set of results for searches that also include the possibility of emission from the $l=2, m=1$ mode at the pulsar's rotation frequency. These are expressed as upper limits on two amplitude parameters $C_{21}$ and $C_{22}$ defined in Jones (2015). We find no strong evidence for gravitational-wave emission from any pulsar in the searches purely for the $l=m=2$ mode, or both the $l=2, m=1,2$ modes.

Further analyses of this data set are possible. For example, we have not presented any updated results regarding potential emission from nontensorial polarization modes as performed in Abbott et al. (2018a). In addition to this, the results from all pulsars could be combined in a way, such as that described in Pitkin et al. (2018), to constrain the underlying pulsar ellipticity distribution and determine whether the ensemble of all pulsars provides evidence for any gravitational-wave signal.

With the MSPs PSR J0636+5129 and PSR J0711-6830 within a factor of $\sim 3$ of their respective spin-down limits, the imminent third observing run of the advanced LIGO and Virgo detectors $(\mathrm{O} 3)$ could allow us to obtain limits below the spindown limit for an MSP for the first time. This offers the intriguing possibility for signal detection from these extremely smooth objects, with spin-down-derived ellipticities of a few $\times 10^{-9}$. The $\mathrm{O} 3$ sensitivity could also bring the limits for the Crab pulsar into the range of mass quadrupoles allowed by reasonably standard neutron star equations of state.

The authors gratefully acknowledge the support of the United States National Science Foundation (NSF) for the construction and operation of the LIGO Laboratory and Advanced LIGO, as well as the Science and Technology Facilities Council (STFC) of the United Kingdom, the Max-Planck-Society (MPS), and the State of Niedersachsen/Germany for support of the construction of Advanced LIGO and construction and operation of the GEO600 detector. Additional support for Advanced LIGO was provided by the Australian Research Council. The authors 
gratefully acknowledge the Italian Istituto Nazionale di Fisica Nucleare (INFN), the French Centre National de la Recherche Scientifique (CNRS), and the Foundation for Fundamental Research on Matter supported by the Netherlands Organisation for Scientific Research, for the construction and operation of the Virgo detector and the creation and support of the EGO consortium. The authors also gratefully acknowledge research support from these agencies, as well as by the Council of Scientific and Industrial Research of India, the Department of Science and Technology, India, the Science \& Engineering Research Board (SERB), India, the Ministry of Human Resource Development, India, the Spanish Agencia Estatal de Investigación, the Vicepresidència i Conselleria d'Innovació Recerca i Turisme and the Conselleria d'Educació i Universitat del Govern de les Illes Balears, the Conselleria d'Educació Investigació Cultura i Esport de la Generalitat Valenciana, the National Science Centre of Poland, the Swiss National Science Foundation (SNSF), the Russian Foundation for Basic Research, the Russian Science Foundation, the European Commission, the European Regional Development Funds (ERDF), the Royal Society, the Scottish Funding Council, the Scottish Universities Physics Alliance, the Hungarian Scientific Research Fund (OTKA), the Lyon Institute of Origins (LIO), the National Research, Development and Innovation Office Hungary (NKFI), the National Research Foundation of Korea, Industry Canada and the Province of Ontario through the Ministry of Economic Development and Innovation, the Natural Science and Engineering Research Council Canada, the Canadian Institute for Advanced Research, the Brazilian Ministry of Science, Technology, Innovations, and Communications, the International Center for Theoretical Physics South American Institute for Fundamental Research (ICTPSAIFR), the Research Grants Council of Hong Kong, the National Natural Science Foundation of China (NSFC), the Leverhulme Trust, the Research Corporation, the Ministry of Science and Technology (MOST), Taiwan, and the Kavli Foundation. The authors gratefully acknowledge the support of the NSF, STFC, MPS, INFN, CNRS, and the State of Niedersachsen/Germany for provision of computational resources.

The Nançay Radio Observatory is operated by the Paris Observatory, associated with the French CNRS. We acknowledge financial support from the "Programme National Gravitation, Références, Astronomie, Métrologie (PNGRAM) and "Programme National Hautes Énergies (PNHE) of CNRS/INSU, France. Work at the Naval Research Laboratory is supported by NASA. We gratefully acknowledge the continuing contributions of the NICER science team in providing up-to-date spin ephemerides for X-ray-bright pulsars of interest to the LVC. NICER is a $0.2-12 \mathrm{keV}$ X-ray telescope operating on the International Space Station. The NICER mission and portions of the NICER science team activities are funded by NASA.

This work has been assigned LIGO document number LIGO-P1800344.

Facilities: Arecibo, Fermi, LIGO, Lovell, Molonglo Observatory, MtPO:26 m, NICER, NRT, Parkes.

Software: Much of the analysis described in the paper was performed using the publicly available LALSUITE library (LIGO Scientific Collaboration 2018). Production of many of the pulsar timing ephemerides used in this analysis was performed with TEMPO $^{206}$ and TEMPO2 (Hobbs et al. 2006b). Figures in this publication have be produced using Matplotlib (Hunter 2007).

206 http://tempo.sourceforge.net/

\section{Appendix A \\ Definitions}

Here we will define some of the standard useful quantities reported and used in our results (many of these are defined in Aasi et al. 2014). The standard definition for the gravitationalwave amplitude from the $l=m=2$ mass quadrupole for a nonprecessing triaxial star rotating about a principal axis is

$$
\begin{aligned}
h_{0} & =\frac{16 \pi^{2} G}{c^{4}} \frac{I_{z z}^{\mathrm{fid}} \varepsilon f_{\mathrm{rot}}^{2}}{d} \approx 4.23 \times 10^{-26}\left(\frac{1 \mathrm{kpc}}{d}\right) \\
& \times\left(\frac{I_{z z}^{\mathrm{fid}}}{10^{38} \mathrm{~kg} \mathrm{~m}^{2}}\right)\left(\frac{\varepsilon}{10^{-6}}\right)\left(\frac{f_{\mathrm{rot}}}{100 \mathrm{~Hz}}\right)^{2},
\end{aligned}
$$

where $d$ is the pulsar distance, $I_{z z}^{\mathrm{fid}}$ is the fiducial component of the moment-of-inertia tensor ellipsoid about the rotation axis, $f_{\text {rot }}$ is the pulsar's rotation frequency, and $\varepsilon$ is the star's fiducial ellipticity (see, e.g., Johnson-McDaniel 2013) defined as

$$
\varepsilon=\frac{\left|I_{x x}-I_{y y}\right|}{I_{z z}^{\mathrm{fid}}},
$$

where $I_{x x}$ and $I_{y y}$ are the true moments of inertia about the principal axes other than the rotation axis.

The gravitational-wave amplitude is related to the $l=m=$ 2 mass quadrupole $Q_{22}$ via

$$
\begin{aligned}
Q_{22} & \equiv I_{z z}^{\mathrm{fid}} \varepsilon \sqrt{\frac{15}{8 \pi}}=h_{0}\left(\frac{c^{4} d}{16 \pi^{2} G f_{\text {rot }}^{2}}\right) \sqrt{\frac{15}{8 \pi}} \approx 1.83 \\
& \times 10^{32}\left(\frac{h_{0}}{10^{-25}}\right)\left(\frac{d}{1 \mathrm{kpc}}\right)\left(\frac{100 \mathrm{~Hz}}{f_{\text {rot }}}\right)^{2} \mathrm{~kg} \mathrm{~m}^{2},
\end{aligned}
$$

where we use the definition of the mass quadrupole used in Owen (2005) and defined in Ushomirsky et al. (2000). Alternatively, we can use $h_{0}$ to calculate the fiducial ellipticity, defined as

$$
\begin{aligned}
\varepsilon= & \frac{h_{0}}{I_{z z}^{\mathrm{fid}}}\left(\frac{c^{4} d}{16 \pi^{2} G f_{\mathrm{rot}}^{2}}\right) \approx 2.36 \times 10^{-6}\left(\frac{h_{0}}{10^{-25}}\right) \\
& \times\left(\frac{d}{1 \mathrm{kpc}}\right)\left(\frac{100 \mathrm{~Hz}}{f_{\text {rot }}}\right)^{2}\left(\frac{10^{38} \mathrm{~kg} \mathrm{~m}^{2}}{I_{z z}^{\mathrm{fid}}}\right) .
\end{aligned}
$$

If emission of gravitational radiation via the $l=m=2$ mass quadrupole is considered to be the sole energy loss mechanism for a pulsar, then by equating the gravitational-wave luminosity (see, e.g., Equation (4) of Aasi et al. 2014)

$$
\begin{aligned}
\dot{E}_{\mathrm{gw}} & =\frac{8 \pi^{2} c^{3}}{5 G} f_{\text {rot }}^{2} h_{0}^{2} d^{2} \approx 6.07 \times 10^{29} \\
& \times\left(\frac{f_{\text {rot }}}{100 \mathrm{~Hz}}\right)^{2}\left(\frac{h_{0}}{10^{-25}}\right)^{2}\left(\frac{d}{1 \mathrm{kpc}}\right)^{2} \mathrm{~W},
\end{aligned}
$$

with the loss of kinetic energy inferred from the the first frequency derivative $\dot{f}_{\text {rot }}$ of the pulsar

$$
\begin{aligned}
\dot{E}_{\mathrm{KE}} & =4 \pi^{2} I_{z z}^{\mathrm{fid}} f_{\text {rot }}\left|\dot{f}_{\text {rot }}\right| \approx 3.95 \times 10^{30} \\
& \times\left(\frac{I_{z z}^{\mathrm{fid}}}{10^{38} \mathrm{~kg} \mathrm{~m}^{2}}\right)\left(\frac{f_{\text {rot }}}{100 \mathrm{~Hz}}\right)\left(\frac{\left|\dot{f}_{\text {rot }}\right|}{10^{-11} \mathrm{~Hz} \mathrm{~s}^{-1}}\right) \mathrm{W},
\end{aligned}
$$


one can define the spin-down limit on $h_{0}$, where

$$
\begin{aligned}
h_{0}^{\mathrm{sd}} & =\frac{1}{d}\left(\frac{5}{2} \frac{G I_{z z}^{\mathrm{fid}}}{c^{3}} \frac{\left|\dot{f}_{\text {rot }}\right|}{f_{\text {rot }}}\right)^{1 / 2} \approx 2.55 \times 10^{-25}\left(\frac{1 \mathrm{kpc}}{d}\right) \\
& \times\left(\frac{I_{z z}^{\mathrm{fid}}}{10^{38} \mathrm{~kg} \mathrm{~m}^{2}}\right)^{1 / 2}\left(\frac{100 \mathrm{~Hz}}{f_{\text {rot }}}\right)^{1 / 2}\left(\frac{\left|\dot{f}_{\mathrm{rot}}\right|}{10^{-11} \mathrm{~Hz} \mathrm{~s}^{-1}}\right)^{1 / 2} .
\end{aligned}
$$

By equating Equations (3) and (9), we can rearrange and get spin-down limits on $Q_{22}$ as

$$
\begin{aligned}
Q_{22}^{\mathrm{sd}} & =\left(\frac{75}{4096 \pi^{5}} \frac{I_{z z}^{\mathrm{fid}} c^{5}}{G} \frac{\dot{f}_{\mathrm{rot}}}{f_{\text {rot }}^{5}}\right)^{1 / 2} \approx 4.66 \times 10^{32} \\
& \times\left(\frac{I_{z z}^{\mathrm{fid}}}{10^{38} \mathrm{~kg} \mathrm{~m}^{2}}\right)^{1 / 2}\left(\frac{100 \mathrm{~Hz}}{f_{\text {rot }}}\right)^{5 / 2}\left(\frac{\left|\dot{f}_{\text {rot }}\right|}{10^{-11} \mathrm{~Hz} \mathrm{~s}^{-1}}\right)^{1 / 2} \mathrm{~kg} \mathrm{~m}^{2}
\end{aligned}
$$

and on $\varepsilon$ as

$$
\begin{aligned}
\varepsilon^{\mathrm{sd}} & =\left(\frac{5}{512 \pi^{4}} \frac{c^{5}}{I_{z z}^{\mathrm{fid}} G} \frac{\dot{f}_{\text {rot }}}{f_{\text {rot }}^{5}}\right)^{1 / 2} \approx 6.03 \times 10^{-6} \\
& \times\left(\frac{10^{38} \mathrm{~kg} \mathrm{~m}^{2}}{I_{z z}^{\mathrm{fid}}}\right)^{1 / 2}\left(\frac{100 \mathrm{~Hz}}{f_{\text {rot }}}\right)^{5 / 2}\left(\frac{\left|\dot{f}_{\text {rot }}\right|}{10^{-11} \mathrm{~Hz} \mathrm{~s}^{-1}}\right)^{1 / 2},
\end{aligned}
$$

where it is interesting to note that these are independent of the distance to the pulsar.

For a triaxial source not rotating about a principal axis, and emitting via both the $l=2, m=1$ and the $l=m=2$ quadrupole modes, the relations between the waveform amplitudes and phases given in Equations (1) and (2) and the source moment-of-inertia tensor components and Euler orientation angle $\theta$ are described in Section 3.1 of Jones (2015). We will not repeat the relationships here, but note that how to convert between the two definitions is described in detail in the Appendix of Pitkin et al. (2015).

\section{Appendix B Priors}

In this appendix we will detail the prior probability distributions used on parameters by the Bayesian and $5 n$-vector analysis methods. The use of these priors for the Bayesian search is discussed in Pitkin et al. (2017), and the motivation behind some of the prior limits used is discussed in Jones (2015) and Pitkin et al. (2015). For the 5n-vector pipeline, priors are set on signal initial phase $\phi_{0}$ and polarization parameters $\psi, \cos \iota$ in the computation of upper limits.

For the gravitational-wave-specific orientation parameters for searches purely from the $l=m=2$ mode, the following priors have been used. ${ }^{207}$ The initial rotational phase of the pulsar at a given epoch $\phi_{0}$, the polarization angle $\psi$, and the cosine of the inclination angle $\cos \iota$ have uniform priors ${ }^{208}$

\footnotetext{
207 In the notation used here $\sim$ stands for "has the probability distribution of," and $\mathcal{U}(a, b)$ is a continuous uniform distribution with a constant probability $1 /(b-a)$ for $x \in[a, b]$.

${ }^{208}$ The polarization angle $\psi$ and orientation angle $\iota$ have a joint prior that is uniform over a sphere, with degeneracies when thinking purely in terms of the gravitational-wave waveforms described in Jones (2015), but these can be reparameterized to independent uniform priors if in terms of $\cos \iota$.
}

given by

$$
\begin{aligned}
\phi_{0} & \sim \mathcal{U}(0, \pi), \\
\psi & \sim \mathcal{U}(0, \pi / 2), \\
\cos \iota & \sim \mathcal{U}(-1,1) .
\end{aligned}
$$

For the Bayesian search, the prior on the gravitationalwave amplitude $h_{0}$ is based on observed upper limits, or sensitivity estimates, from previous LIGO and Virgo runs. The form of the prior is given by a Fermi-Dirac-type probability distribution (see, e.g., that used in Middleton et al. 2016) as described in Pitkin et al. (2017), which has a flat region followed by an exponential decay region but is nonzero for all positive values. It is defined as

$$
\begin{aligned}
& p(x \mid \sigma, \mu, I) \\
& =\left\{\begin{array}{cl}
\frac{1}{\sigma \ln \left(1+e^{\mu / \sigma}\right)}\left(e^{(x-\mu) / \sigma}+1\right)^{-1} & \text { if } x \geqslant 0, \\
0 & \text { otherwise, }
\end{array}\right.
\end{aligned}
$$

where $\mu$ gives the value at which the distribution decays to $50 \%$ of its maximum value and $\sigma$ controls the width of the band over which the bulk of the decay happens. The band around $\mu$ over which the probability density falls from $97.5 \%$ to $2.5 \%$ of its peak value is given by $\mu \pm 7.33 \mu / 2 r$, where $r=\mu / \sigma$. In our case we specify that this fall-off happens over a range that is $40 \%$ of the value of $\mu$, so that $r=7.33 /(2 \times 0.4)=9.1625$. The value of $\mu$ is set by finding the value that produces a specific bound within which $95 \%$ of the probability is constrained (bounded by zero at the lower end) given the previous value of $r$. The specific bound is that based on the sensitivity for each pulsar (i.e., the $95 \%$ upper limits on $h_{0}$; see Appendix C) that would have been expected if using data from the sixth LIGO science run and fourth Virgo science run, scaled up by a factor of 25 to be conservative and make sure that the likelihood is well within the flat part of the prior distribution, while disfavoring arbitrarily large values. ${ }^{209}$

For the searches that include both the $l=2, m=1,2$ modes the phase and orientation angle priors have been given by

$$
\begin{aligned}
\Phi_{21}^{C} & \sim \mathcal{U}(0,2 \pi), \\
\Phi_{22}^{C} & \sim \mathcal{U}(0,2 \pi), \\
\psi & \sim \mathcal{U}(0, \pi / 2), \\
\cos \iota & \sim \mathcal{U}(-1,1) .
\end{aligned}
$$

As discussed above, in the Bayesian method the priors on the amplitude parameters $C_{21}$ and $C_{22}$ have used Fermi-Dirac probability distributions for which the parameters have been set in the same way as done for $h_{0}$. However, in this case the sensitivity estimate used for $h_{0}$ is assumed to be valid for $C_{21}$ and $C_{22}$, while in reality there are factors of a few differences. These differences are allowable given the scaling factor used and the sensitivity improvements over S6.

In our searches we make use of the pulsar rotational phase parameters (frequency, frequency derivatives, sky location, proper motion, and Keplerian and relativistic binary system

\footnotetext{
209 A discussion about a choice between a uniform prior and a uniform in logarithm prior for the amplitude parameter is given in Appendix B of Isi et al. (2017).
} 
orbital parameters if relevant) derived from electromagnetic observation of pulse times of arrival. These parameters are obtained by fitting the phase model to the times of arrival using software such as TEMPO2 Hobbs et al. (2006b) to produce ephemeris files, and these fits include uncertainty estimates. In most cases, and where it is computationally feasible, for any combination of parameters in the ephemeris files that have been refit (i.e., a new estimate has been performed using data that matched the requirements of our search, such as being concurrent with the LIGO observing runs) we include a multivariate Gaussian prior in our analysis, for which the diagonal of the covariance matrix is derived from the uncertainties in the ephemeris file and taking them to be one standard deviation values. In the prior covariance matrix we assume no correlations between parameters except in two pairs of cases for pulsars in binary systems; for very low eccentricity systems $(e<0.001)$ with refitted uncertainties on both the time and angle of periastron, or with refitted values on the period and time derivative of the angle of periastron, the covariance matrix is set such as to make these pairs fully correlated.

As described in Abbott et al. (2010, 2017a) and Aasi et al. (2014), there are some pulsars for which we can place tighter constraints on their orientation. In particular, the inclination angle and gravitational-wave polarization angle can be assumed to be measured by modeling X-ray observations of their surrounding pulsar wind nebulae (Ng \& Romani 2004, 2008). In this analysis, for PSR J0205+6449, PSR J0534+2200, PSR J0835-4510, PSR J1952+3252, and PSR J2229+6114, in addition to a search using the above priors, we also perform parameter estimation using the restricted priors given in Table 3 of Abbott et al. (2017a), based on values taken from Ng \& Romani (2008). In these cases the priors are on the inclination angle $\iota$ rather than its cosine. The prior probability distribution on $\psi$ is a unimodal Gaussian, but that on $\iota$ is given by the sum of a pair of Gaussian distributions with different means, which is required to account for the fact that rotation directions of the stars are unknown (Jones 2015).
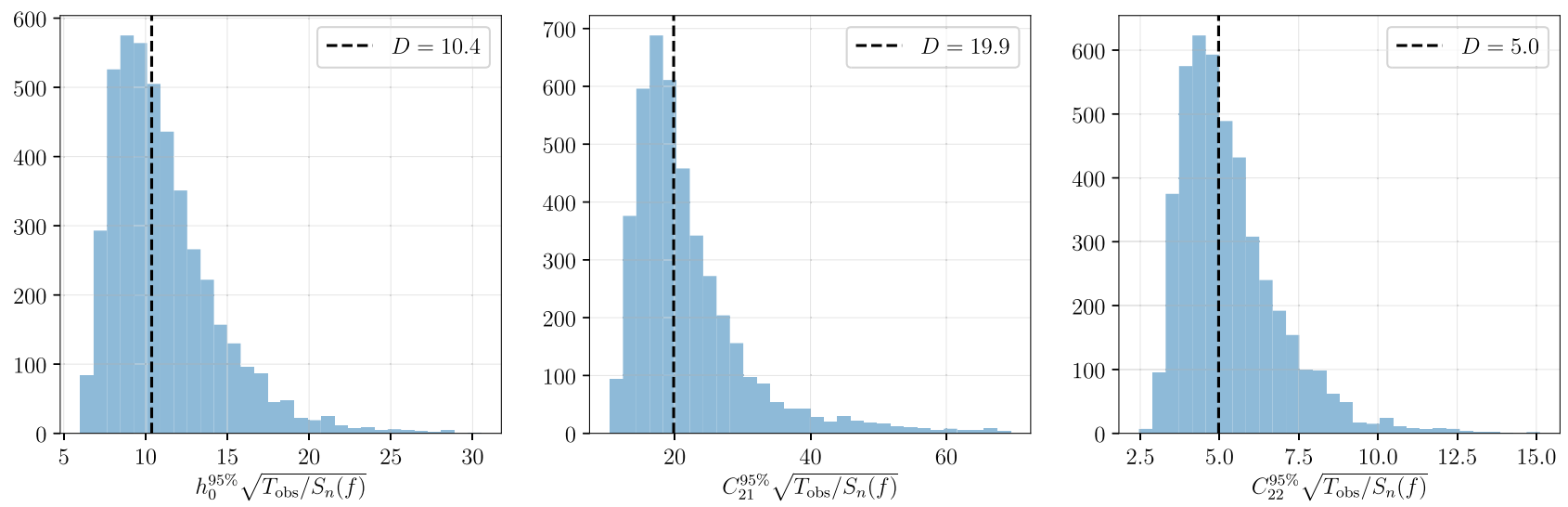

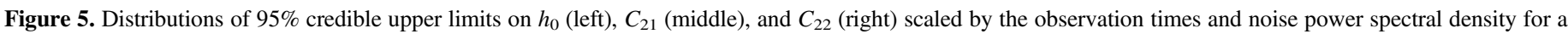

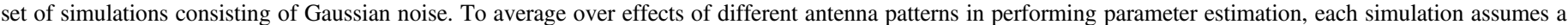
random source sky location for a uniform distribution over the sky. 


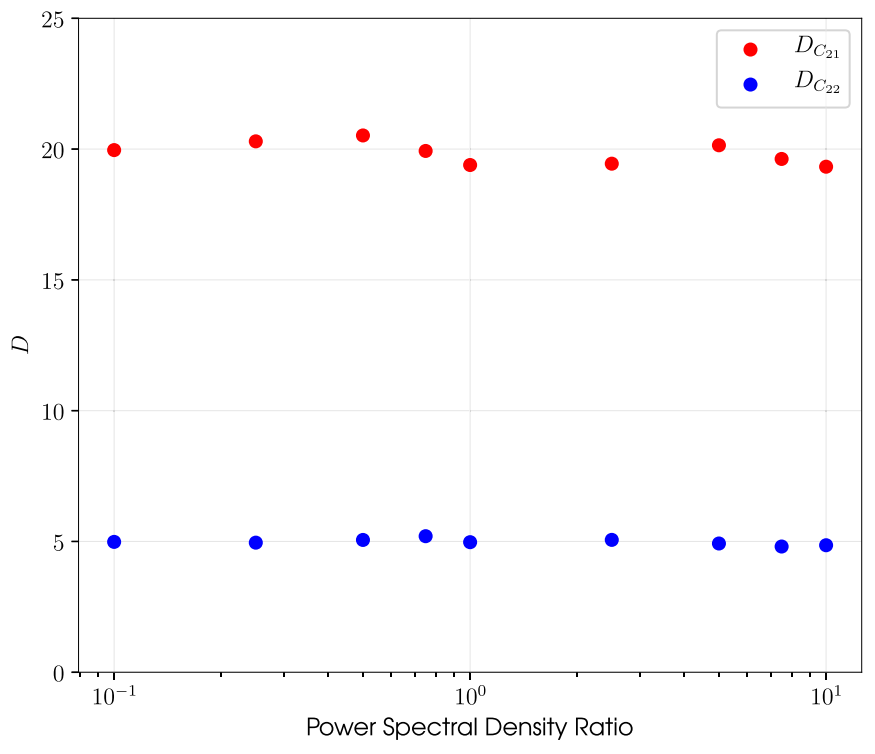

Figure 6. $D$ scale factor for the $C_{21}$ and $C_{22}$ upper limits as a function of the power spectral density ratio between the data at equivalents of the rotation frequency and twice the rotation frequency.

To estimate the sensitivity to the $C_{21}$ and $C_{22}$ amplitude parameters for an $l=2, m=1,2$ mode search, we have performed similar simulations to those described above. A search including both modes is not completely independent for each mode, as there are common orientation parameters. Hence, we also wanted to investigate whether the sensitivity at either amplitude is affected by the noise level at the other amplitude. We generated simulations consisting of independent Gaussian noise in two data streams: one equivalent to the data at the rotation frequency and another equivalent to the data at twice the rotation frequency. For the data stream at twice the rotation frequency the noise was always drawn from a Gaussian distribution with the same variance defined by a power spectral density of $10^{-48} \mathrm{~Hz}^{-1 / 2}$. For the data stream at the rotation frequency we created multiple sets of 500 instantiations where the noise was drawn from a Gaussian distribution with a variance defined by a power spectral density of $10^{-48} x \mathrm{~Hz}^{-1 / 2}$, where for each set of $500 x$ was a different factor between 0.1 and 10. The $D$ scale factor from Equation (13) for both the $C_{21}$ and $C_{22}$ amplitude upper limit for each set of 500 simulations and as a function of $x$ is shown in Figure 6. It can be seen that there is no obvious correlation between the power spectral density ratio $x$ and the value of $D$, which suggests that the upper limits on the two amplitudes are actually largely independent.

We see from Figures 5 and 6 that the value of $D$ used to estimate the sensitivity for $C_{21}$ is 19.9 , and the value of $D$ used to estimate the sensitivity for $C_{22}$ is 5.0. These values have been used when producing the sensitivity curves in Figure 1.

\section{Appendix D \\ Mixed Bayesian/Frequentist Upper Limit Computation for the $5 n$-vector Method}

Given a measured value $S^{*}$ of a detection statistic $\mathcal{S}$, the frequentist upper limit at a given confidence level $\alpha$ is defined as that value of signal amplitude $h_{\mathrm{ul}}$ such that a signal with amplitude $h_{0}>h_{\mathrm{ul}}$ produces a value of the detection statistic bigger than $S^{*}$ in a fraction $\alpha$ of a large number of repeated experiments: $P\left(S>S^{*} \mid h_{0}>h_{\mathrm{ul}}\right)=\alpha$. Typically, the upper limit is computed using Neyman's rule for the construction of confidence intervals (Neyman 1937). This classical frequentist upper limit has the following well-known and unpleasant feature: if the value of the detection statistic $S^{*}$ falls in the first $1-\alpha$ quantile of its noise-only distribution, the resulting upper limit is exactly zero. This behavior, although legitimate in the frequentist framework, poses a problem, for instance, when upper limits obtained in the analysis of data sets with different sensitivity are compared. It may happen that, due to a noise fluctuation, the upper limit set for the more noisy data is below that computed for the less noisy one. This kind of problem may happen also for Bayesian upper limits, but it is exacerbated in the classical frequentist case.

The unwanted features of the classical Neyman's construction have been overcome in the Feldman-Cousins unified approach, where, using the freedom inherent in Neyman's construction, a method to obtain a unified set of classical confidence intervals for computing both upper limits and twosided confidence intervals has been obtained (Feldman \& Cousins 1998). The Feldman-Cousins approach sometimes is difficult to implement and, similarly to Neyman's approach, does not allow accounting for nonuniform prior distributions for nuisance parameters.

We have developed an alternative method for setting upper limits on signal amplitude that keeps the advantages of the frequentist approach, like the ease of implementation and computational speed, while avoiding its problems. The basic idea is that of computing the posterior distribution of the signal amplitude conditioned to the measured value of the detection statistic. The main steps of the procedure can be summarized as follows.

We consider a set of possible signal amplitudes $H_{0}$. For each amplitude we generate several signals with polarization parameters distributed according to given prior distributions, and for each signal we compute the corresponding value of the detection statistic. Hence, the probability distribution of the detection statistic, for the different signal amplitudes, can be built; see Figure 7.

For each distribution we determine the value corresponding to the measured detection statistic $p\left(S^{*} \mid H_{0}\right)$. By multiplying each value by the prior probability density of the signal amplitude, $p\left(H_{0}\right)$, and normalizing, we obtain the posterior probability distribution for the signal amplitude: $p\left(H_{0} \mid S^{*}\right) \propto$ $p\left(S^{*} \mid H_{0}\right) p\left(H_{0}\right)$; see Figure 8. 


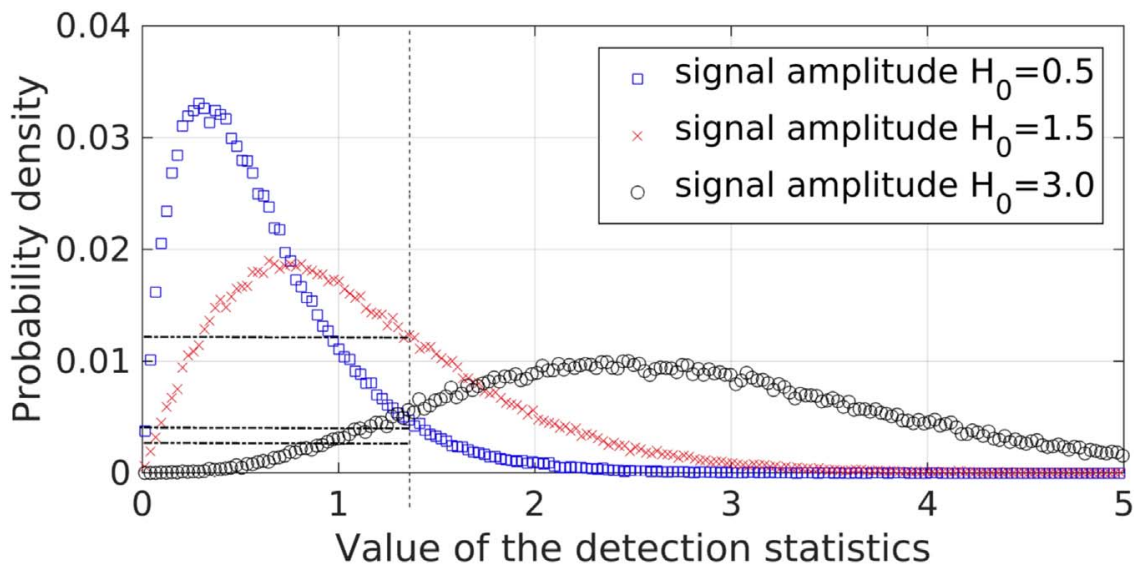

Figure 7. Probability distributions of the detection statistic $\mathcal{S}$ after having injected into Gaussian noise with $\sigma=1$ signals with three different amplitudes. Given the measured value of the detection statistic $\mathcal{S}^{*}$ (shown by the vertical dashed line), the corresponding values of probability density for the various signal amplitudes are determined (shown by the horizontal dot-dashed lines).

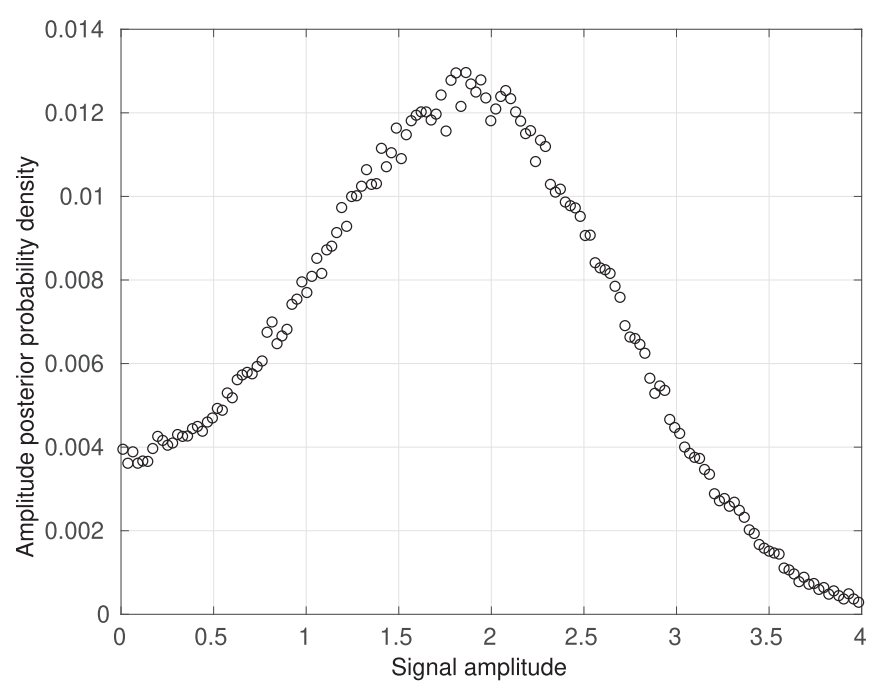

Figure 8. Posterior probability distribution of the signal amplitude for the given measured value $\mathcal{S}^{*}$ of the detection statistic.

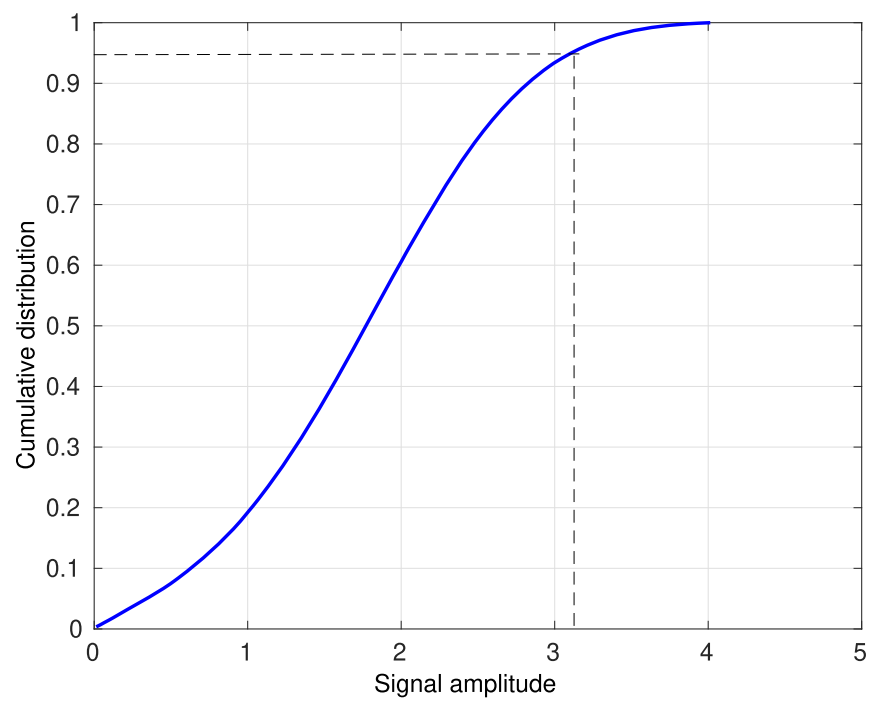

Figure 9. Cumulative posterior probability distribution of the signal amplitude. The amplitude value corresponding to $95 \%$ of the cumulative is the wanted credible upper limit.
We then calculate the cumulative probability distribution and obtain the amplitude value corresponding to a given probability, e.g., 0.95; see Figure 9. This is the 95\% credible upper limit.

\section{Appendix E}

\section{Amplitude Conversion Factors for the $\mathbf{5 n}$-vector Method}

The $5 n$-vector method uses a nonstandard formalism to describe the gravitational-wave signal, based on the concept of polarization ellipse (Astone et al. 2010; Abadie et al. 2011; Aasi et al. 2014). In this formalism the signal strain is given by the real part of

$$
h(t)=H_{0}\left(H_{+} \boldsymbol{e}^{+}+H_{\times} \boldsymbol{e}^{\times}\right) e^{l\left(\omega_{0}(t) t+\Phi_{0}\right)},
$$

where $\omega_{0}(t)$ is the signal angular frequency, $\boldsymbol{e}^{+/ \times}$are the two basis polarization tensors, $\Phi_{0}$ is the signal phase at the time $t=0$, and the two complex amplitudes $H_{+}, H_{\times}$are given by

$$
H_{+}=\frac{\cos 2 \psi-\imath \eta \sin 2 \psi}{\sqrt{1+\eta^{2}}}, H_{\times}=\frac{\sin 2 \psi+\imath \eta \cos 2 \psi}{\sqrt{1+\eta^{2}}},
$$

in which $\eta \in[-1,1]$ is the ratio of the polarization ellipse semiminor to semimajor axis and the polarization angle $\psi$ defines, as usual, the direction of the major axis with respect to the celestial parallel of the source (measured counterclockwise). The signal described by Equation (15) is general, i.e., does not assume any specific emission mechanism by a spinning neutron star. Assuming a triaxial star spinning about a principal axis of inertia, the overall amplitude $H_{0}$ is related to the standard $h_{0}$ by

$$
h_{0}=\frac{2 H_{0}}{\sqrt{1+6 \cos ^{2} \iota+\cos ^{4} \iota}} .
$$

For the emission at the star's rotational frequency of the $l=2$, $m=1$ harmonic mode (see Equation (1)), the relation between $H_{0}$ and the amplitude $C_{21}$ is given by

$$
C_{21}=\frac{2 H_{0}}{\sqrt{1-\cos ^{4} \iota}} \text {. }
$$

As discussed in, e.g., Aasi et al. (2014), upper limits are computed on $H_{0}$ and then converted to $h_{0}$ or $C_{21}$ using Equations (17) and (18), where the functions of $\iota$ are replaced by their mean value: $h_{0}^{95 \%} \simeq 1.37 H_{0}^{95 \%}$ and $C_{21}^{95 \%} \simeq 1.31 H_{0}^{95 \%}$. 


\section{References}

Aasi, J., Abadie, J., Abbott, B. P., et al. 2014, ApJ, 785, 119

Aasi, J., Abbott, B. P., Abbott, R., et al. 2015a, CQGra, 32, 074001

Aasi, J., Abbott, B. P., Abbott, R., et al. 2015b, PhRvD, 91, 022004 Abadie, J., Abbott, B. P., Abbott, R., et al. 2011, ApJ, 737, 93 Abbott, B., Abbott, R., Adhikari, R., et al. 2004, PhRvD, 69, 082004 Abbott, B., Abbott, R., Adhikari, R., et al. 2005, PhRvL, 94, 181103 Abbott, B., Abbott, R., Adhikari, R., et al. 2007, PhRvD, 76, 042001 Abbott, B., Abbott, R., Adhikari, R., et al. 2008, ApJL, 683, L45 Abbott, B. P., Abbott, R., Abbott, T. D., et al. 2016, PhRvL, 116, 131103 Abbott, B. P., Abbott, R., Abbott, T. D., et al. 2017a, ApJ, 839, 12 Abbott, B. P., Abbott, R., Abbott, T. D., et al. 2017b, PhRvD, 96, 122006 Abbott, B. P., Abbott, R., Abbott, T. D., et al. 2017c, PhRvL, 119, 161101 Abbott, B. P., Abbott, R., Abbott, T. D., et al. 2017d, PhRvL, 119, 141101 Abbott, B. P., Abbott, R., Abbott, T. D., et al. 2018a, PhRvL, 120, 031104 Abbott, B. P., Abbott, R., Abbott, T. D., et al. 2018b, PhRvL, 121, 161101 Abbott, B. P., Abbott, R., Abbott, T. D., et al. 2019, arXiv:1902.08442 Abbott, B. P., Abbott, R., Acernese, F., et al. 2010, ApJ, 713, 671 Abbott, B. P., Abbott, R., Adhikari, R., et al. 2009, RPPh, 72, 076901 Accadia, T., Acernese, F., Alshourbagy, M., et al. 2012, JInst, 7, 3012 Acernese, F., Agathos, M., Agatsuma, K., et al. 2015, CQGra, 32, 024001 Akgün, T., Link, B., \& Wasserman, I. 2006, MNRAS, 365, 653 Antoniadis, J., Freire, P. C. C., Wex, N., et al. 2013, Sci, 340, 448 Arzoumanian, Z., Brazier, A., Burke-Spolaor, S., et al. 2018, ApJS, 235, 37 Arzoumanian, Z., Nice, D. J., Taylor, J. H., \& Thorsett, S. E. 1994, ApJ, 422, 671 Ashton, G., Jones, D. I., \& Prix, R. 2015, PhRvD, 91, 062009

Ashton, G., Jones, D. I., \& Prix, R. 2017, MNRAS, 467, 164

Astone, P., Colla, A., D’Antonio, S., Frasca, S., \& Palomba, C. 2012, JPhCS, 363 , 012038

Astone, P., D'Antonio, S., Frasca, S., \& Palomba, C. 2010, CQGra, 27, 194016 Bassa, C. G., Antoniadis, J., Camilo, F., et al. 2016, MNRAS, 455, 3806 Behnke, B., Papa, M. A., \& Prix, R. 2015, PhRvD, 91, 064007 Bejger, M. 2013, A\&A, 552, A59

Bejger, M., \& Królak, A. 2014, CQGra, 31, 105011

Bogdanov, S., Ho, W. C. G., Enoto, T., et al. 2019, ApJ, 877, 69

Bonazzola, S., \& Gourgoulhon, E. 1996, A\&A, 312, 675

Boyles, J., Lynch, R. S., Ransom, S. M., et al. 2013, ApJ, 763, 80

Braga, V. F., Dall'Ora, M., Bono, G., et al. 2015, ApJ, 799, 165

Cahillane, C., Betzwieser, J., Brown, D. A., et al. 2017, PhRvD, 96, 102001

Cahillane, C., Hulko, M., Kissel, J. S., et al. 2018, LIGO G1800319, https:// dcc.ligo.org/LIGO-G1800319/public

Caplan, M. E., Schneider, A. S., \& Horowitz, C. J. 2018, PhRvL, 121, 132701 Cordes, J. M. 1993, in ASP Conf. Ser. 36, Planets around Pulsars, ed. J. A. Phillips, S. E. Thorsett, \& S. R. Kulkarni (San Francisco, CA: ASP), 43 Cordes, J. M., \& Helfand, D. J. 1980, ApJ, 239, 640

Cordes, J. M., \& Lazio, T. J. W. 2002, arXiv:astro-ph/0207156

Cutler, C. 2002, PhRvD, 66, 084025

Damour, T., \& Taylor, J. H. 1991, ApJ, 366, 501

Davis, D., Massinger, T. J., Lundgren, A. P., et al. 2019, CQGra, 36, 055011

Deller, A. T., Bailes, M., \& Tingay, S. J. 2009, Sci, 323, 1327

Deller, A. T., Boyles, J., Lorimer, D. R., et al. 2013, ApJ, 770, 145

Desvignes, G., Caballero, R. N., Lentati, L., et al. 2016, MNRAS, 458, 3341

Dodson, R., Legge, D., Reynolds, J. E., \& McCulloch, P. M. 2003, ApJ, 596, 1137

Driggers, J. C., Vitale, S., Lundgren, A. P., et al. 2019, PhRvD, 99, 042001

Dupuis, R. J., \& Woan, G. 2005, PhRvD, 72, 102002

Durant, M., Kargaltsev, O., Pavlov, G. G., Kropotina, J., \& Levenfish, K. 2013 , ApJ, 763, 72

Espinoza, C. M., Guillemot, L., Çelik, Ö., et al. 2013, MNRAS, 430, 571

Espinoza, C. M., Lyne, A. G., Stappers, B. W., \& Kramer, M. 2011, MNRAS, 414,1679

Fattoyev, F. J., Horowitz, C. J., \& Lu, H. 2018, arXiv:1804.04952

Feldman, G. J., \& Cousins, R. D. 1998, PhRvD, 57, 3873

Ferdman, R. D., Stairs, I. H., Kramer, M., et al. 2014, MNRAS, 443, 2183

Fonseca, E., Stairs, I. H., \& Thorsett, S. E. 2014, ApJ, 787, 82

Freire, P. C. C., Abdo, A. A., Ajello, M., et al. 2011, Sci, 334, 1107

Freire, P. C. C., Wex, N., Esposito-Farèse, G., et al. 2012, MNRAS, 423, 3328

Gotthelf, E. V., Halpern, J. P., Terrier, R., \& Mattana, F. 2011, ApJL, 729, L16

Gratton, R. G., Bragaglia, A., Carretta, E., et al. 2003, A\&A, 408, 529

Halpern, J. P., Bogdanov, S., \& Gotthelf, E. V. 2013, ApJ, 778, 120

Halpern, J. P., Gotthelf, E. V., Leighly, K. M., \& Helfand, D. J. 2001, ApJ, 547,323

Harris, W. E. 1996, yCat, 7195, 0
Hobbs, G., Lyne, A., \& Kramer, M. 2006a, ChJAA, 6, 169

Hobbs, G., Lyne, A. G., Kramer, M., Martin, C. E., \& Jordan, C. 2004, MNRAS, 353, 1311

Hobbs, G. B., Edwards, R. T., \& Manchester, R. N. 2006b, MNRAS, 369, 655

Horowitz, C. J. 2010, PhRvD, 81, 103001

Hunter, J. D. 2007, CSE, 9, 90

Isi, M., Pitkin, M., \& Weinstein, A. J. 2017, PhRvD, 96, 042001

Jaranowski, P., \& Królak, A. 2010, CQGra, 27, 194015

Jaranowski, P., Królak, A., \& Schutz, B. F. 1998, PhRvD, 58, 063001

Johnson, T. J., Guillemot, L., Kerr, M., et al. 2013, ApJ, 778, 106

Johnson-McDaniel, N. K. 2013, PhRvD, 88, 044016

Johnson-McDaniel, N. K., \& Owen, B. J. 2013, PhRvD, 88, 044004

Johnston, S., Hobbs, G., Vigeland, S., et al. 2005, MNRAS, 364, 1397

Jones, D. I. 2004, PhRvD, 70, 042002

Jones, D. I. 2010, MNRAS, 402, 2503

Jones, D. I. 2012, MNRAS, 420, 2325

Jones, D. I. 2015, MNRAS, 453, 53

Jones, D. I., \& Andersson, N. 2002, MNRAS, 331, 203

Jones, D. I., Ashton, G., \& Prix, R. 2017, PhRvL, 118, 261101

Keith, M. J., Johnston, S., Weltevrede, P., \& Kramer, M. 2010, MNRAS, 402, 745

Kothes, R. 2013, A\&A, 560, A18

Kramer, M., \& Johnston, S. 2008, MNRAS, 390, 87

LIGO Scientific Collaboration 2018, LIGO Algorithm Library, Caltech Library, doi:10.7935/GT1W-FZ16

Manchester, R. N., Hobbs, G. B., Teoh, A., \& Hobbs, M. 2005, AJ, 129, 1993

Marelli, M., Harding, A., Pizzocaro, D., et al. 2014, ApJ, 795, 168

Mastrano, A., \& Melatos, A. 2012, MNRAS, 421, 760

McNamara, B. J., Harrison, T. E., \& Baumgardt, H. 2004, ApJ, 602, 264

Melatos, A., \& Phinney, E. S. 2001, PASA, 18, 421

Middleton, H., Del Pozzo, W., Farr, W. M., Sesana, A., \& Vecchio, A. 2016, MNRAS, 455, L72

Mingarelli, C. M. F., Anderson, L., Bedell, M., \& Spergel, D. N. 2018, arXiv: 1812.06262

Neyman, J. 1937, RSPTA, 236, 333

Ng, C., Bailes, M., Bates, S. D., et al. 2014, MNRAS, 439, 1865

Ng, C.-Y., \& Romani, R. W. 2004, ApJ, 601, 479

Ng, C.-Y., \& Romani, R. W. 2008, ApJ, 673, 411

Ortolani, S., Barbuy, B., Bica, E., Zoccali, M., \& Renzini, A. 2007, A\&A, 470,1043

Ostriker, J. P., \& Gunn, J. E. 1969, ApJ, 157, 1395

Owen, B. J. 2005, PhRvL, 95, 211101

Palfreyman, J. 2016, ATel, 9847, 1

Palfreyman, J., Dickey, J. M., Hotan, A., Ellingsen, S., \& van Straten, W. 2018, Natur, 556, 219

Palomba, C. 2000, A\&A, 354, 163

Payne, D. J. B., \& Melatos, A. 2004, MNRAS, 351, 569

Pitkin, M., Gill, C., Jones, D. I., Woan, G., \& Davies, G. S. 2015, MNRAS, 453, 4399

Pitkin, M., Isi, M., Veitch, J., \& Woan, G. 2017, arXiv:1705.08978v1

Pitkin, M., Messenger, C., \& Fan, X. 2018, PhRvD, 98, 063001

Pitkin, M., \& Woan, G. 2007, PhRvD, 76, 042006

Reardon, D. J., Hobbs, G., Coles, W., et al. 2016, MNRAS, 455, 1751

Rees, R. F., \& Cudworth, K. M. 1991, AJ, 102, 152

Romani, R. W., \& Shaw, M. S. 2011, ApJL, 743, L26

Shklovskii, I. S. 1970, SvA, 13, 562

Spiewak, R., Bailes, M., Barr, E. D., et al. 2018, MNRAS, 475, 469

Stairs, I. H., Lyne, A. G., Kramer, M., et al. 2019, MNRAS, 485, 3230

Ushomirsky, G., Cutler, C., \& Bildsten, L. 2000, MNRAS, 319, 902

Valenti, E., Ferraro, F. R., \& Origlia, L. 2007, AJ, 133, 1287

Valenti, E., Ferraro, F. R., \& Origlia, L. 2010, MNRAS, 402, 1729

Vallisneri, M., Kanner, J., Williams, R., Weinstein, A., \& Stephens, B. 2015 , JPhCS, 610, 012021

Verbiest, J. P. W., \& Lorimer, D. R. 2014, MNRAS, 444, 1859

Verbiest, J. P. W., Weisberg, J. M., Chael, A. A., Lee, K. J., \& Lorimer, D. R. 2012, ApJ, 755, 39

Vigeland, S. J., Deller, A. T., Kaplan, D. L., et al. 2018, ApJ, 855, 122

Wang, W. 2011, RAA, 11, 824

Woan, G., Pitkin, M. D., Haskell, B., Jones, D. I., \& Lasky, P. D. 2018, ApJL, 863, L40

Worley, A., Krastev, P. G., \& Li, B.-A. 2008, ApJ, 685, 390

Yao, J. M., Manchester, R. N., \& Wang, N. 2017, ApJ, 835, 29

Zimmermann, M. 1980, PhRvD, 21, 891

Zimmermann, M., \& Szedenits, E., Jr. 1979, PhRvD, 20, 351 


\title{
New Star Observations with NuSTAR: Flares from Young Stellar Objects in the $\rho$ Ophiuchi Cloud Complex in Hard X-Rays
}

\author{
Juliana T. Vievering ${ }^{1}$ (D), Lindsay Glesener ${ }^{1}$ (D), Brian W. Grefenstette ${ }^{2}$ (D), and David M. Smith ${ }^{3}$ (D) \\ ${ }^{1}$ School of Physics \& Astronomy, University of Minnesota, Twin Cities, Minneapolis, MN 55455, USA; vieve004@umn.edu \\ ${ }^{2}$ Cahill Center for Astronomy and Astrophysics, California Institute of Technology, Pasadena, CA 91125, USA \\ ${ }^{3}$ Santa Cruz Institute for Particle Physics and Department of Physics, University of California, Santa Cruz, CA 95064, USA \\ Received 2018 October 25; revised 2019 June 7; accepted 2019 June 26; published 2019 September 4
}

\begin{abstract}
We study the structure and dynamics of extreme flaring events on young stellar objects (YSOs) observed in hard X-rays by the Nuclear Spectroscopic Telescope Array (NuSTAR). During 2015 and 2016, NuSTAR made three observations of the star-forming region $\rho$ Ophiuchi, each with an exposure $\sim 50 \mathrm{ks}$. NuSTAR offers unprecedented sensitivity above $\sim 7 \mathrm{keV}$, making this data set the first of its kind. Through improved coverage of hard X-rays, it is finally possible to directly measure the high-energy thermal continuum for hot plasmas and to sensitively search for evidence of nonthermal emission from YSO flares. During these observations, multiple flares were observed, and spectral and timing analyses were performed on three of the brightest flares. By fitting an optically thin thermal plasma model to each of these events, we found flare plasma heated to high temperatures $(\sim 40-80 \mathrm{MK})$ and determined that these events are $\sim 1000$ times brighter than the brightest flares observed on the Sun. Two of the studied flares showed excess emission at $6.4 \mathrm{keV}$, and this excess may be attributable to iron fluorescence in the circumstellar disk. No clear evidence for a nonthermal component was observed, but upper limits on nonthermal emission allow for enough nonthermal energy to account for the estimated thermal energy in the flare on protostar IRS 43, which is consistent with the standard model for solar and stellar flares.
\end{abstract}

Unified Astronomy Thesaurus concepts: Star forming regions (1565); Stellar flares (1603); Stellar x-ray flares (1637); Pre-main sequence stars (1290); Young stellar objects (1834); Stellar activity (1580)

\section{Introduction}

Observed flares on distant stars are typically assumed to be similar to flares on our own Sun. Standard models for solar flares theorize that these energetic events are driven by magnetic reconnection, and during this process, a significant portion of the dissipated magnetic energy $(\sim 40 \%)$ is converted into kinetic energy of particles (e.g., Aschwanden et al. 2016). These accelerated particles then travel along magnetic field lines, producing nonthermal bremsstrahlung emission through interactions with dense chromospheric plasma (Brown 1971) and heating the ambient plasma to high temperatures $(>10 \mathrm{MK})$. This heated plasma then expands into the flare loop in a process called chromospheric evaporation and produces thermal bremsstrahlung emission. In this model, both thermal and nonthermal processes result in emission of X-rays, and thus, spectroscopic measurements in the X-ray regime are key to understanding the nature of energy release and transfer in flares.

When studying stellar flares, young stellar objects (YSOs) are particularly interesting targets as their heightened magnetic activity leads to extreme flaring events. The term YSO covers the early stages of a star's life, from infalling protostar $\left(\sim 10^{4} \mathrm{yr}\right)$ to weak-lined T Tauri stars $\left(\sim 10^{7} \mathrm{yr}\right)$. From early infrared-millimeter observations of YSOs, Lada \& Wilking (1984) developed an evolutionary classification system (Class I through Class III) based on characteristics of spectral energy distributions in this waveband, with higher class numbers corresponding to more evolved YSOs (Wilking \& Lada 1983).

Along with hosting extreme flaring events, YSOs also prove to be interesting sources due to the presence of circumstellar disks, which allows for the possibility of different flare loop configurations, such as photosphere-disk and disk-disk, in addition to the photospheric footpoints for flares on solar-type and M dwarf stars (Feigelson \& Montmerle 1999). Though the dense circumstellar material associated with YSOs strongly attenuates emission in certain wavebands, including the optical, higher-energy emission in the X-ray regime can be transmitted and measured by X-ray observatories.

Observations of intense X-ray flares on YSOs can additionally provide an opportunity to investigate the impact of high-energy radiation on the surrounding environment. One major question regarding YSOs is whether their flaring activity has an impact on planet formation. If enough high-energy $\mathrm{X}$-ray emission penetrates the protoplanetary disk, it is possible that the disk material could become sufficiently ionized to lead to magnetorotational instabilities (MRIs; Feigelson 2010).

YSOs have previously been observed in the X-ray regime by observatories such as Chandra and XMM-Newton (see Imanishi et al. 2001; Pillitteri et al. 2010). Surveys of the nearby starforming region $\rho$ Ophiuchi ( $\sim 120 \mathrm{pc}$; Loinard et al. 2008) by both observatories have detected many YSO flares and found through spectral analyses, that Class I sources are associated with hotter temperatures and larger absorption columns than their older counterparts. Additionally, these surveys have led to the discovery of interesting spectral features, such as the first detected $6.4 \mathrm{keV}$ line from a Class I source, which has been attributed to iron fluorescence (Imanishi et al. 2001). However, due to limited sensitivity at higher X-ray energies, these studies do not measure or place constraints on nonthermal emission, which is essential for understanding the energy transfer from nonthermal electrons to heating of plasma.

The Nuclear Spectroscopic Telescope Array $(N u S T A R)$ is the first satellite to use focusing optics in the hard X-ray regime and overtakes the effective are of previous X-ray imaging observatories above $\sim 6-7 \mathrm{keV}$. With improved 
Table 1

NuSTAR Observations of $\rho$ Ophiuchi

\begin{tabular}{lccc}
\hline \hline Sequence ID & Start Date and Time (UT) & End Date and Time (UT) & Exposure (ks) \\
\hline 30102028002 & 2015-May 10 10:31:07 & 2015-May-11 14:51:07 & 55 \\
30102028004 & 2015-Aug-25 18:56:08 & 2015-Aug-26 23:36:08 & 51 \\
30102028006 & 2016-Apr-29 09:36:08 & 2016-Apr-30 11:11:08 & 46 \\
\hline
\end{tabular}

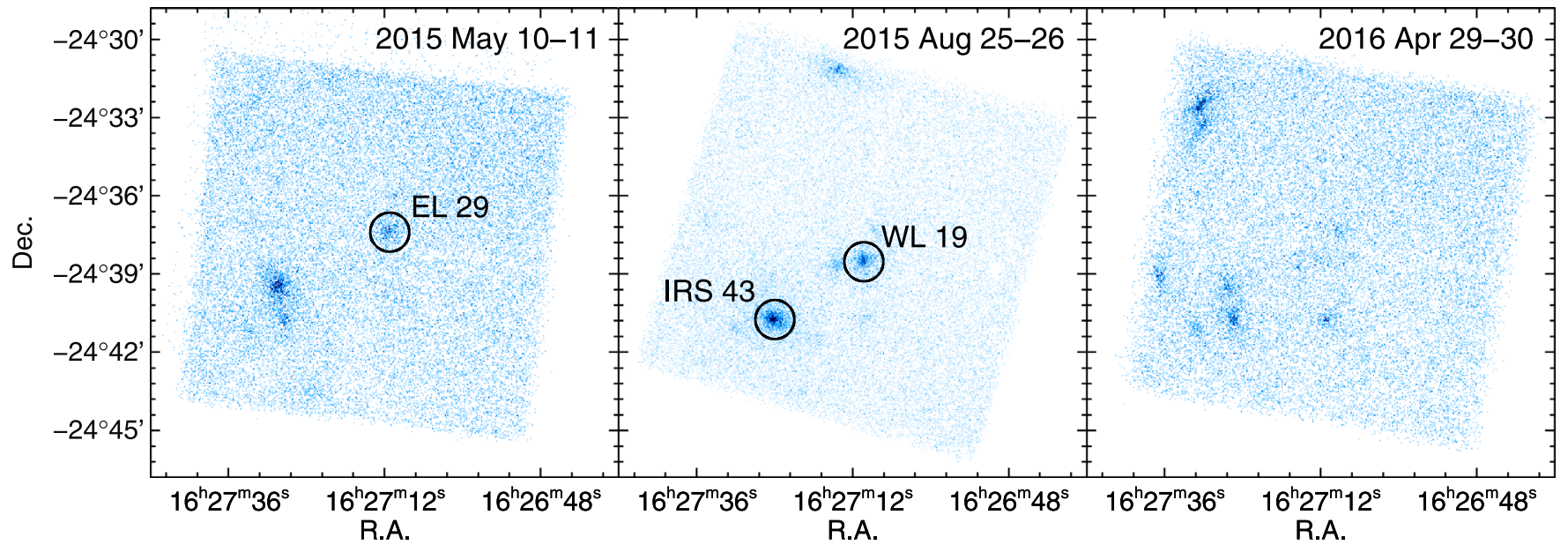

Figure 1. Images from FPMA show the time integrated NuSTAR observations of $\rho$ Ophiuchi over the whole FOV and the full energy range of 3-79 keV. Images are not background subtracted.

coverage of higher-energy X-rays, it is possible to search for evidence of nonthermal emission, to directly measure thermal continuum of hot plasmas, and to investigate the impact of high-energy radiation on circumstellar disks. NUSTAR performed the first focused hard X-ray $(\gtrsim 10 \mathrm{keV})$ observations of YSOs during 2015 and 2016 through three $\sim 50 \mathrm{ks}$ exposures of $\rho$ Ophiuchi. Over the course of these observations, NUSTAR observed multiple X-ray flares from YSOs, and the brightest of these events are analyzed here. Section 2 introduces the observations and outlines the process for data reduction. In Section 3, the analysis of flare spectra is described, and the corresponding results are presented. Section 4 offers a discussion of the results, and Section 5 provides a summary of our study.

\section{Observations and Data Processing}

The star-forming region $\rho$ Ophiuchi was observed by NuSTAR over three $\sim 50 \mathrm{ks}$ exposures during 2015 and 2016 as part of NuSTAR's Guest Observer Program (see Table 1).The NuSTAR science instrument is composed of two grazing incidence telescopes that are optimized over the energy range of 3-79 keV (Harrison et al. 2013). Each focal plane module, focal plane module (FPMA) and focal plane module B (FPMB), contains a $2 \times 2$ array of pixellated cadmium zinc telluride detectors, leading to small crosshair gaps in the image. Data from FPMA/FPMB were processed using the NuSTAR data analysis software (NuSTARDAS ${ }^{4}$ v1.6.0).

The flares analyzed here were selected by eye from full fieldof-view (FOV) images integrated over the entire observation period (see Figure 1). Multiple distinct flares were observed during the three observing intervals, and three of these flares have been analyzed in depth (see Figure 2 for corresponding

\footnotetext{
4 https://heasarc.gsfc.nasa.gov/docs/nustar/analysis/nustar_swguide.pdf
}
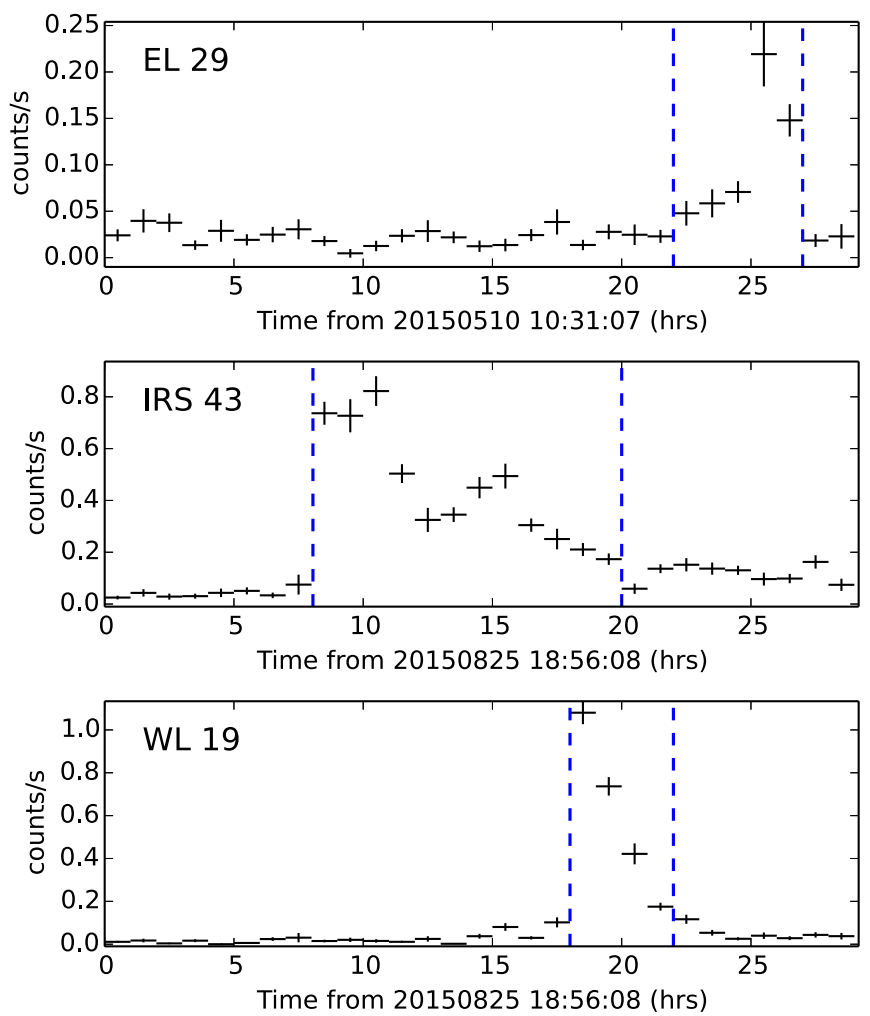

Figure 2. Light curves (binned by hour) of three YSO flares during the first two NuSTAR observations of $\rho$ Ophiuchi with combined data from FPMA and FPMB over the full energy range of 3-79 keV. Dashed lines indicate the time interval selected for the flare spectral analysis.

light curves). Sources were identified by comparing the flare location with catalogs from previous surveys of $\rho$ Ophiuchi (Imanishi et al. 2001; Pillitteri et al. 2010). During the first 
observation, Class I protostar Elias 29 (hereafter EL 29; Elias 1978) produced a flare that lasted $\sim 5 \mathrm{hr}$. Two large flares occurred during the second observation: one from Class I protostar IRS 43 (Wilking et al. 1989) and one from Class III source WL 19 (Wilking \& Lada 1983). These flares lasted $\sim 12.6$ and $\sim 4.2 \mathrm{hr}$, respectively. In future analyses, we will both examine the bright additional flares visible in Figure 1 and search for other potential sources near the sensitivity limits of NUSTAR.

\section{Analysis}

\subsection{Background Estimation}

The relatively low background of NUSTAR $\left(\sim 10^{-3}\right.$ counts $\mathrm{s}^{-1}$ at $10-30 \mathrm{keV}$ ) within the half-power diameter includes focused cosmic X-ray background (CXB), unfocused CXB through the open light path, environmental neutrons, and instrument background (e.g., fluorescence lines). The backgrounds for the sources studied here were simulated through use of the nuskybgd suite of IDL routines (Wik et al. 2014). In this method, a source-free region of the FOV is selected-in our case, an annulus around each flaring YSO; each background component has a known spectral shape, and a fit of the normalizations of these components is performed based on the selected background region. Once this fit is performed, the background is determined for the whole FOV, and we can estimate the background at the source position.

\subsection{Spectral Analysis}

For each flaring source, a time frame was selected by eye to encompass the rise through decline of the flare (see Figure 2). The source extraction region was reduced to a circular region with a radius of $15^{\prime \prime}$ centered on the source. Counts outside the calibrated NUSTAR energy range (3-79 keV) were removed prior to the analysis, and spectra were binned so that each bin included a minimum of 30 counts. The high end of the energy range for the spectral analysis was further limited by low statistics (i.e., not enough counts at higher energies to make a bin with at least 30 counts), and most spectra extend up to $\sim 20 \mathrm{keV}$. The spectral analysis was performed for each source in XSPEC (version 12.9.0u), simultaneously fitting data from both FPMA and FPMB (see Figure 3). EL 29 falls close to the chip gap for FPMA (closer than for FPMB) during this observation, which is likely the cause of the difference in normalization between the focal plane modules for this spectrum.

\subsubsection{Single Temperature Model}

For each source, the flare data were modeled as an optically thin thermal plasma (vapec) with an absorption component (tbabs) to account for attenuation by circumstellar material, which is mainly important at lower X-ray energies. The free parameters for this model, labeled " $1-\mathrm{T}+\mathrm{abs}$ " in Table 2, included temperature $(k T)$, absorption column $\left(N_{\mathrm{H}}\right)$, and a normalization factor $(n)$. From this normalization, we compute an emission measure (EM) by using the
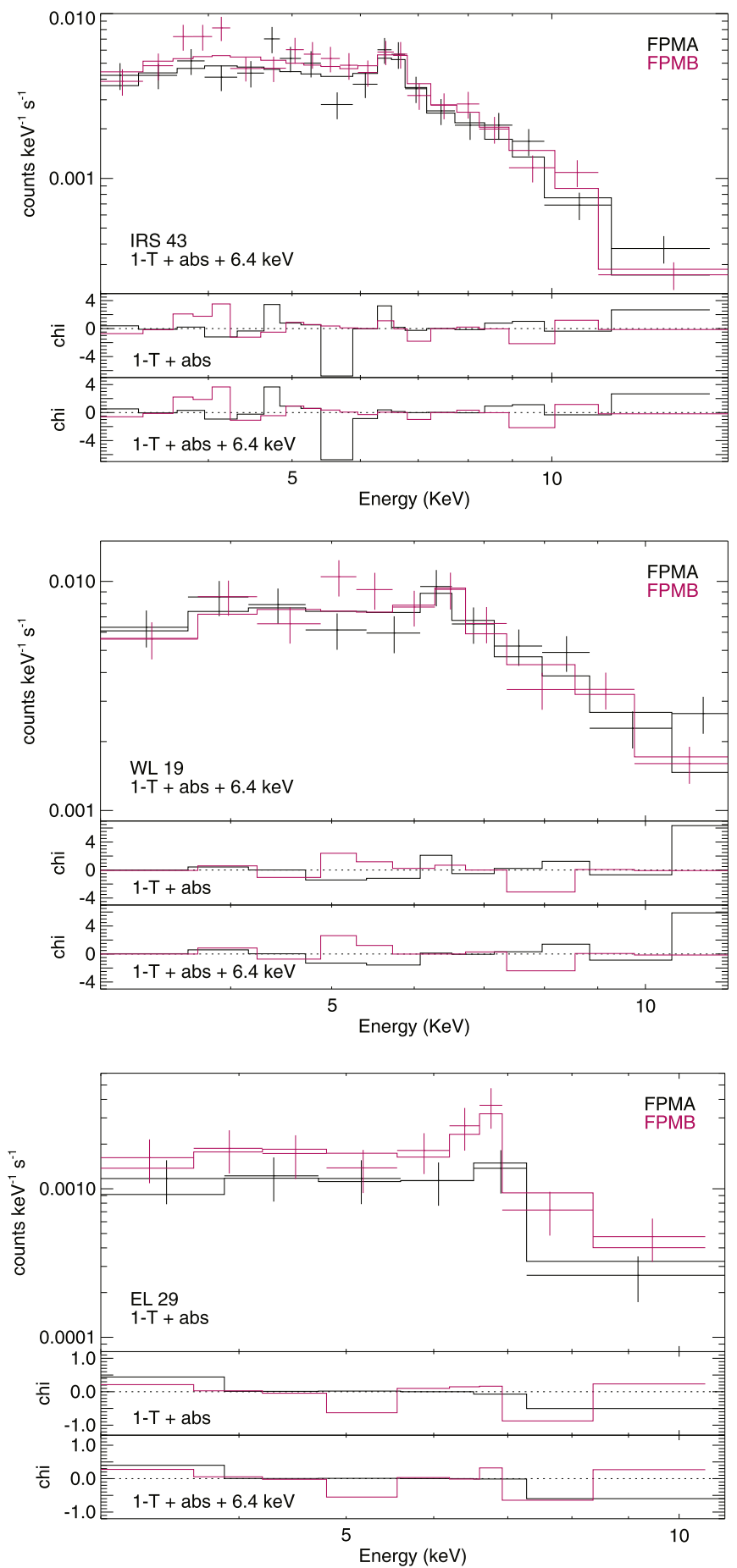

Figure 3. Flare spectra for (top) IRS 43, (middle) WL 19, and (bottom) EL 29. The top panel of each plot shows data from FPMA (black) and FPMB (magenta) along with the best-fit model. Data from FPMA and FPMB are simultaneously fit, with all parameters tied together except for a cross-normalization factor. EL 29 falls close to the chip gap for FPMA (closer than for FPMB) during this observation, which is likely the cause of the difference in normalization between the focal plane modules for this spectrum. The middle panel shows the contribution to the chi-squared value, with sign according to the difference of the data and the model for each data point using an optically thin thermal plasma model (vapec) plus an absorption component (tbabs). With this model, excess emission is observed around $6.4 \mathrm{keV}$ for IRS 43 and WL 19 (but not for EL 29). The bottom panel shows the contribution to the chi-squared value for each data point when a $6.4 \mathrm{keV}$ line is added to the model. For the flares in IRS 43 and WL 19, including this additional emission line improves the fit. 
Table 2

Flare Model Parameters from the Spectral Analysis Described in Section 3.2

\begin{tabular}{|c|c|c|}
\hline Parameters & $1-\mathrm{T}+\mathrm{abs}$ & $1-\mathrm{T}+\mathrm{abs}+6.4 \mathrm{keV}$ \\
\hline \multicolumn{3}{|c|}{ IRS 43} \\
\hline$N_{\mathrm{H}}^{\mathrm{a}}\left(10^{22} \mathrm{~cm}^{-2}\right)$ & $2.3_{0.0}^{4.9}$ & $2.3^{\mathrm{b}}$ \\
\hline$T^{\mathrm{c}}(\mathrm{MK})$ & $57_{50}^{68}$ & $58_{53}^{65}$ \\
\hline$Z_{\mathrm{Fe}}{ }^{\mathrm{d}}$ & $0.2_{0.1}^{0.4}$ & $0.2_{0.0}^{0.3}$ \\
\hline$n_{A}^{\mathrm{e}}\left(10^{-3} \mathrm{~cm}^{-5}\right)$ & $7.7_{5.9}^{9.8}$ & $7.5_{6.6}^{8.5}$ \\
\hline$\chi_{\text {red }}^{2}$ & 1.2 & 1.1 \\
\hline $\mathbf{E M}^{\mathrm{f}}\left(10^{54} \mathrm{~cm}^{-3}\right)$ & $1.3_{1.0}^{1.7}$ & $1.3_{1.1}^{1.5}$ \\
\hline $\mathbf{E} \mathbf{W}^{\mathrm{g}}(\mathbf{e V})$ & $\cdots$ & 190 \\
\hline \multicolumn{3}{|c|}{ WL 19} \\
\hline$N_{\mathrm{H}}\left(10^{22} \mathrm{~cm}^{-2}\right)$ & $3.4_{0.0}^{7.7}$ & $3.4^{\mathrm{b}}$ \\
\hline$T(\mathrm{MK})$ & $76_{57}^{113}$ & $81_{65}^{102}$ \\
\hline$Z_{\mathrm{Fe}}$ & $0.2_{0.0}^{0.5}$ & $0.1_{0.0}^{0.3}$ \\
\hline$n_{A}\left(10^{-3} \mathrm{~cm}^{-5}\right)$ & $9.8_{6.7}^{14.5}$ & $9.4_{7.9}^{11.3}$ \\
\hline$\chi_{\text {red }}^{2}$ & 1.4 & 1.3 \\
\hline EM $\left(10^{54} \mathrm{~cm}^{-3}\right)$ & $1.7_{1.2}^{2.5}$ & $1.6_{1.4}^{1.9}$ \\
\hline $\mathbf{E W}(\mathbf{e V})$ & $\cdots$ & 230 \\
\hline \multicolumn{3}{|c|}{ EL 29} \\
\hline$N_{\mathrm{H}}\left(10^{22} \mathrm{~cm}^{-2}\right)$ & $7.6^{\mathrm{h}}$ & $7.6^{\mathrm{h}}$ \\
\hline$T(\mathrm{MK})$ & $37_{28}^{52}$ & $38_{28}^{54}$ \\
\hline$Z_{\mathrm{Fe}}$ & $0.6_{0.2}^{1.0}$ & $0.5_{0.1}^{1.0}$ \\
\hline$n_{B}^{\mathrm{i}}\left(10^{-3} \mathrm{~cm}^{-5}\right)$ & $4.3_{2.7}^{6.6}$ & $4.2_{2.5}^{6.4}$ \\
\hline$\chi_{\text {red }}^{2}$ & 0.6 & 0.6 \\
\hline EM $\left(10^{54} \mathrm{~cm}^{-3}\right)$ & $0.7_{0.5}^{1.1}$ & $0.7_{0.4}^{1.1}$ \\
\hline EW (eV) & $\ldots$ & 120 \\
\hline
\end{tabular}

Notes. Subscripts and superscripts on the parameter values indicate the lower and upper limits to the $90 \%$ confidence interval, respectively. Bolded values are those derived from fit parameters.

${ }^{\mathrm{a}} N_{\mathrm{H}}$ : hydrogen column density.

${ }^{\text {b }} N_{\mathrm{H}}$ : fixed to fit value from $1 \mathrm{~T}+$ abs model.

${ }^{\mathrm{c}} T$ : temperature.

${ }^{\mathrm{d}} Z_{\mathrm{Fe}}$ : iron abundance relative to solar (Anders \& Grevesse 1989).

e $n_{A}$ : normalization for FPMA data.

${ }^{\mathrm{f}} \mathrm{EM}$ : emission measure, the distance to the source is $\sim 120 \mathrm{pc}$.

${ }^{\mathrm{g}} \mathrm{EW}$ : equivalent width of $6.4 \mathrm{keV}$ emission line.

${ }^{\text {h }} N_{\mathrm{H}}$ : fixed using best-fit value from Imanishi et al. (2001).

${ }^{\mathrm{i}} n_{B}$ : normalization for FPMB data, used instead of $n_{A}$ due to the chip gap issue noted in Section 3.2.

normalization formula for vapec described in the XSPEC manual $^{5}$ and accounting for the distance to the source-in this case, $\sim 120$ pc (Loinard et al. 2008).

In addition, the vapec model allows for a number of elemental abundances to vary with respect to solar abundances, which provides greater flexibility for working with the differing compositions of young stars. In our model, all lighter elements were fixed to solar abundances (Anders \& Grevesse 1989) while the abundance for iron $\left(Z_{\mathrm{Fe}}\right)$, which is an element affecting our energy range and that is typically less abundant for younger stars (Maggio et al. 2007), was allowed to vary. Data from FPMA and FPMB were simultaneously fit, with all parameters tied between data sets apart from a cross-normalization factor. Fit parameters are shown in Table 2 and spectra are shown in Figure 3.

\footnotetext{
5 https://heasarc.gsfc.nasa.gov/xanadu/xspec/manual/node133. html\#vapec
}

\subsection{2. $6.4 \mathrm{keV}$ Emission Line}

After applying an optically thin thermal plasma model, which already includes an iron emission line at $\sim 6.7 \mathrm{keV}$ from the thermal plasma, the flare spectra for IRS 43 and WL 19 showed excess emission around $6.4 \mathrm{keV}$ (see Figure 3). To account for this excess, we added a Gaussian emission line centered at $6.4 \mathrm{keV}$ with $\sigma=0.1 \mathrm{keV}$ (both fixed) and a normalization parameter that was left free. We note that the flux in the $6.4 \mathrm{keV}$ line will, in a sense, trade off with the iron abundance in the vapec model since our coarse energy binning and $N U S T A R$ 's finite resolution will allow the 6.4 and the 6.7 keV lines to share flux in the bins around 6-7 keV.

Since $N_{\mathrm{H}}$ is not well-constrained in our energy range, we fix this parameter to the value from the initial fit (IRS 43 and WL 19) or to a value found in previous studies of the same sources (EL 29); this reduction of free parameters allows for the normalization of the $6.4 \mathrm{keV}$ line, and hence the equivalent width (EW), to be constrained. Best values for the $\mathrm{EW}$, computed in XSPEC, are shown in Table 2. Upon initial comparison with the "1T+abs" model, the addition of a $6.4 \mathrm{keV}$ line to the model slightly improves the fit for the flares on IRS 43 and WL 19, while there is no improvement in the fit quality for the flare observed on EL 29. Even so, for all sources studied, the uncertainty on the EW is large enough to be consistent with zero, so though some of the fits are suggestive of a possible line, the line is not statistically significant. We note that, though we did not find evidence for a $6.4 \mathrm{keV}$ line for EL 29, observations of EL 29 by Chandra and XMM-Newton have yielded positive detections of the $6.4 \mathrm{keV}$ line, during both flaring and quiescent times (Favata et al. 2005; Giardino et al. 2007).

\subsection{GOES Class}

These YSO flares are clearly very bright since they can be observed from $120 \mathrm{pc}$ away. A common measure for flare magnitude when classifying solar flares is the X-ray intensity of the flare peak as observed by the Geostationary Observational Environmental Satellite (GOES) series of Earth-orbiting spacecraft, ${ }^{6}$ which is then classified from faintest to brightest as GOES class A, B, C, M, or X (X-class $>10^{-4} \mathrm{~W} \mathrm{~m}^{-2}$ ). From our isothermal fits, we can estimate what the GOES flux $\left(\mathrm{W} \mathrm{m} \mathrm{m}^{-2}\right.$ ) would be if an event of a certain temperature and emission measure were to occur at a distance of 1 au from Earth. For the YSO flares presented here, the equivalent GOES class ranges from $\mathrm{X} 10^{4}-10^{5}$, which is roughly 1000 times the classification of the largest solar flare on record (Kane et al. 2005).

\subsection{Neupert Effect}

The physical processes behind these flares can be further understood by considering the time evolution of the X-ray emission. We examined the flares for evidence of the Neupert effect, which describes a relationship in which the nonthermal (higher-energy) X-ray output traces the rate of input of thermal plasma from the footpoints to the flare loop, over timescales shorter than the loop cooling time (Neupert 1968; Veronig et al. 2002).

In order to study this, the light curve of the WL 19 flare was split into three energy bands, including a low- (3-6 keV),

\footnotetext{
6 www.swpc.noaa.gov/products/goes-X-ray-flux
} 


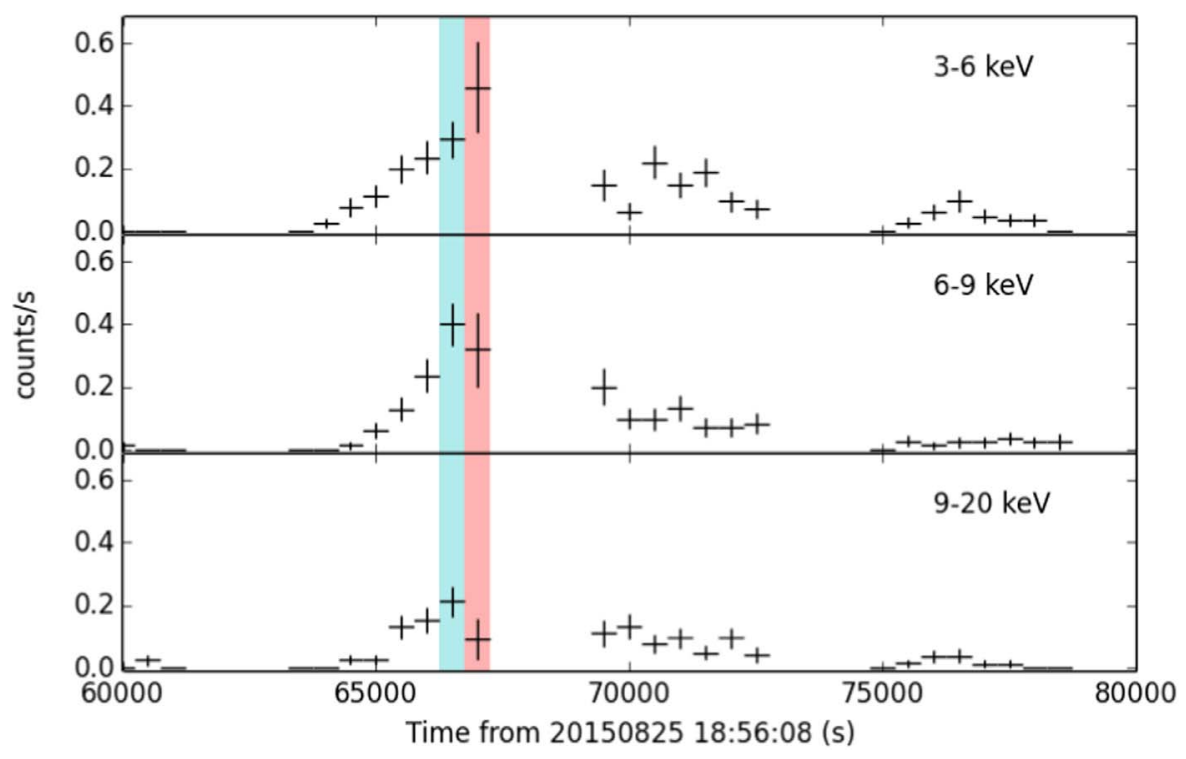

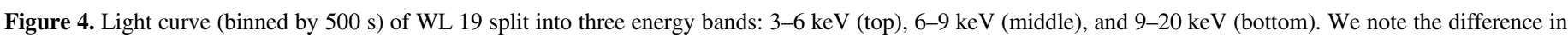

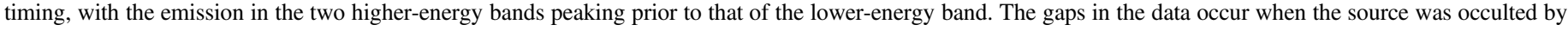
the Earth.

medium- (6-9 keV), and high-energy band (9-20 keV), as seen in Figure 4. From these light curves, we note the difference in peak time between bands, with the two higher-energy bands peaking $\sim 500 \mathrm{~s}$ (one time bin) prior to the lowest energy peak. This effect was not studied in depth for the other two sources due to low statistics and, in the case of IRS 43, gaps in the data because of the source being occulted by the Earth during the rising interval of the flare.

\section{Discussion}

Spectral analysis of three NuSTAR YSO flares, each lasting $15-45 \mathrm{ks}$, found high-temperature plasma ( 40-80 MK) and revealed that these flares are $\sim 1000$ times brighter than the brightest flares on the Sun. An isothermal model effectively described the data for each flare, and no clear evidence for a nonthermal component was found, although the flux at higher energies does peak earlier for the flare on WL 19 (Figure 4). Two of the observed flares, those from IRS 43 and WL 19, showed evidence of an additional emission line at $6.4 \mathrm{keV}$, which is typically attributed to iron fluorescence (discussed further in Section 4.2).

By comparison, our temperatures are found to be consistent with the range of flare temperatures observed in the surveys of $\rho$ Ophiuchi by Chandra (Imanishi et al. 2003) and XMMNewton (Pillitteri et al. 2010). The flares we studied are on the brighter end of those observed in these surveys, which is consistent with our selection method of choosing the brightest events. Similar to our analysis, the Chandra study considered individual flares (in addition to quiescent measurements), and the flare durations are found to be of the same order, averaging $\sim 10-20 \mathrm{ks}$.

\subsection{Flare Energetics}

When considering the energetics of these flares, one thing to address is whether there could be enough energy in nonthermal electrons to account for the observed heating, despite having no clear detection of a nonthermal component above the thermal spectrum. This scenario would allow for a model similar to the standard model for solar flares (Brown 1971) in which energetic electrons deposit energy in the footpoints and heat the ambient plasma, leading to chromospheric evaporation and subsequent thermal emission. The flare on IRS 43 is selected for this energetics analysis since it has the best counting statistics of the sources studied in this paper when integrating over the whole flaring period $(>12 \mathrm{hr}$ ).

\subsubsection{Nonthermal Electron Energy}

With the flares studied so far, a high-temperature plasma model is sufficient to account for emission at high energies, and we see no clear evidence of nonthermal emission. By assuming that these flares are similar to those observed on the Sun and other stars, scaling laws were used to explore whether or not $N U S T A R$ would be sensitive enough to observe nonthermal emission for flares of the observed magnitudes.

In Battaglia et al. (2005), correlations between parameters for a broad population of solar flares, from GOES class A to class M (soft X-ray flux $\sim 10^{-8}-10^{-4} \mathrm{~W} \mathrm{~m}^{-2}$ ), were studied. By using a scaling relation between the maximum GOES flux and nonthermal flux, we estimated the nonthermal NuSTAR flux at $35 \mathrm{keV}$ to be $\sim 1.4 \times 10^{-6}$ counts $\mathrm{keV}^{-1} \mathrm{~s}^{-1}$ for the flare on IRS 43. In addition, a similar scaling law from Isola et al. (2007) was used to estimate the peak nonthermal NuSTAR flux over the energy range $20-40 \mathrm{keV}$. Along with solar flares, that study also includes a number of stellar flares that are closer in magnitude to the ones observed here by $N u S T A R$. This scaling law estimates the peak nonthermal flux from $20-40 \mathrm{keV}$ to be $\sim 3.5 \times 10^{-5}$ counts $\mathrm{keV}^{-1} \mathrm{~s}^{-1}$. When comparing to the NUSTAR background near this energy range, $\sim 10^{-5}$ counts $\mathrm{keV}^{-1} \mathrm{~s}^{-1}$, we note that the nonthermal flux estimates for IRS 43 are either of the order of or below the NUSTAR background. With the very limited statistics above $20 \mathrm{keV}$ for our observed flares, it is plausible that there could be an undetected nonthermal component among the background. Thus, the absence of a clear nonthermal component to the model does not eliminate the possibility that YSO flares follow the standard model for solar flares. In addition, we are optimistic that NUSTAR may be able to detect a nonthermal 


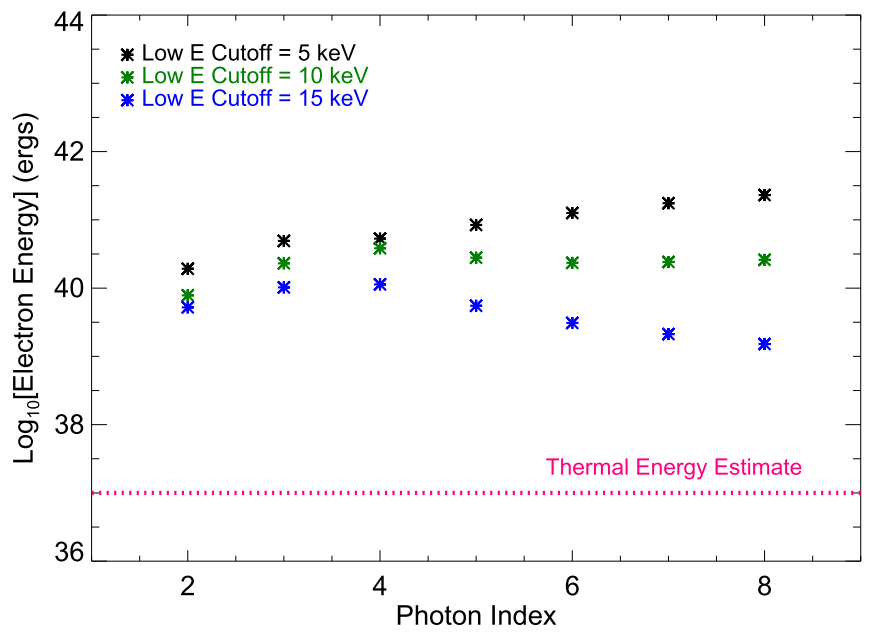

Figure 5. Estimated upper limits on nonthermal electron energy for the flare on IRS 43 over a range of photon indices and cutoff energies of $5 \mathrm{keV}$ (black), $10 \mathrm{keV}$ (green), and $15 \mathrm{keV}$ (blue). For each scenario, the upper limit to the nonthermal electron energy far exceeds the estimated thermal energy (magenta dotted line), indicating that the energy in an undetected nonthermal electron population, if present, could plausibly account for the thermal energy in the flare.

component for larger magnitude flares in future observations of YSOs.

In a separate line of reasoning, we consider how large of a nonthermal component could be present and undetected within our current model. To test this, we add a model to our spectral analysis of IRS 43 that represents nonthermal bremsstrahlung emission-in this case, a broken power-law model (bknpower). Using the thick-target model described in Brown (1971), the parameters from a broken power law in the photon spectrum can be translated into a corresponding electron spectrum with a spectral index, $\delta$, and a low-energy cutoff, $E_{c}$, from which the nonthermal electron energy can be calculated.

For the broken power-law model, the index below an assumed break energy ${ }^{7}\left(E_{\text {break }} \leqslant E_{c}\right)$ is set to $\gamma_{1}=1$, and we test a series of photon indices above $E_{\text {break }}$ at integer intervals from $\gamma_{2}=2$ to $\gamma_{2}=8$. The photon index $\gamma_{2}$ corresponds to the electron spectral index $\delta$ such that $\delta=\gamma_{2}+1$ for a thick-target model. For each $\gamma_{2}$, all parameters of the broken power-law model are fixed, and the normalization is raised until the quality of the fit to the data is affected, ${ }^{8}$ i.e., until the chi-squared value increases by a certain amount. In order to be conservative in our estimate of the plausible nonthermal energy available in the flare, we only allowed for a small increase in chi-squared, ${ }^{9}$ corresponding to a $5 \%$ increase in the confidence with which the fit can be rejected; allowing for additional degradation of the fit quality by raising the normalization further would only increase the amount of nonthermal energy available. With these model parameters, we determine the electron spectrum, test a range of $E_{c}$ values, and compute a rough upper limit on the nonthermal electron energy in the flare (see results in Figure 5). From this plot, we find that the upper limit to the nonthermal

\footnotetext{
$E_{\text {break }}$ is set to $5 \mathrm{keV}$ for $E_{c}=5 \mathrm{keV}$ and set to $10 \mathrm{keV}$ for $E_{c}=10 \mathrm{keV}$ and $E_{c}=15 \mathrm{keV}$.

${ }^{8}$ For other model components (e.g., isothermal plasma, absorption, etc.), the same parameters are left free, as described in Section 3.2.

9 We allowed for an increase in chi-squared from 37.65 to 38.97 (dof $=34$ ), which corresponds to a $5 \%$ increase in the confidence with which the fit can be rejected.
}

electron energy ranges from $\sim 10^{39}-10^{41} \mathrm{erg}$. We note that some combinations of parameters even improved the quality of the fit; the setting an index of $\gamma_{2}=2(\delta=3)$ and a normalization of $\sim 6 \times 10^{-5}$ photons $\mathrm{keV}^{-1} \mathrm{~cm}^{2} \mathrm{~s}^{-1}$ (at $1 \mathrm{keV}$ ) resulted in the best fit of the parameters tested. These parameters correspond to $E_{\mathrm{NT}} \sim(3-4) \times 10^{39} \mathrm{erg}$, depending on the value of $E_{c}$ used.

Given the limited sensitivity of previous X-ray instrumentation above $\sim 10 \mathrm{keV}$, estimates of nonthermal energy for stellar flares in the literature are currently lacking, particularly for YSOs. Studies on surveys of star-forming regions by chargecoupled device (CCD)-based instruments, such as the observations of $\rho$ Ophiuchi by Chandra and XMM-Newton, do not make attempts to place limits on a nonthermal component. A study by Osten et al. (2007), which analyzes a superflare observed by the Swift X-Ray Telescope (XRT) and Burst Alert Telescope (BAT) on the active binary II Pegasi (II Peg), states that it is the first study to provide evidence for a nonthermal component during a stellar flare. Though this is an inherently different flare on a different stellar source, it is nonetheless interesting to note their findings when considering trends for large magnitude events. In this study of II Peg, the best-fit electron spectral indices for two time intervals are $\delta \sim 2.8$ and $\delta \sim 3.1$, which are similar in hardness to the fixed index in our study corresponding to an improved overall fit quality $(\delta=3)$. These findings for extreme stellar events are consistent with patterns observed for solar flares, where a correlation is found between higher nonthermal flux and lower (harder) electron spectral indices (Battaglia et al. 2005). The energetics analysis in Osten et al. (2007) estimates a total electron energy that exceeds the thermal radiative losses; however, challenges with constraining conductive losses in this study make it difficult to state conclusively whether the nonthermal energy can account for total thermal energy in the flare.

\subsubsection{Thermal Energy}

To determine if the estimated upper limits to the nonthermal energy are sufficient to account for the thermal energy, we estimate both radiative and conductive losses for the flare on IRS 43. For an estimate of the radiative losses, we take our thermal model for this flare and extend the model to a broad range of energies in XSPEC. We considered the range of $0.01-200 \mathrm{keV}$, which is a typical energy range for radiation from the corona that has been used in previous stellar X-ray studies (Audard et al. 1999; Osten et al. 2007). By integrating the spectrum over this range, we obtain a radiative flux (erg $\left.\mathrm{cm}^{-2} \mathrm{~s}^{-1}\right)$; the total radiative energy can be computed by accounting for the duration of the flare $(\Delta t)$ and the distance to the source $(D)$. For the observed flare on IRS $43(\Delta t \sim 21 \mathrm{ks}$, $\left.D \sim 3.7 \times 10^{20} \mathrm{~cm}\right)$, the estimated radiated energy released in the corona is $E_{\text {rad }} \sim 6 \times 10^{35} \mathrm{erg}$. We note that this is a lower limit to the radiative losses; multiple studies of solar flares indicate that a majority of the total radiated flare energy comes from the visible and infrared wavebands (Woods et al. 2004, 2006). The actual value for thermal energy could be five times larger than our estimate, bringing the possible value for radiative losses up to $\sim 3 \times 10^{36} \mathrm{erg}$.

In addition to radiative losses, a flare study by Warmuth $\&$ Mann (2016) has also found conductive losses to be significant 
in flares. Conductive energy losses can be computed by

$$
E_{\text {cond }}=\frac{\kappa T^{7 / 2} \mathrm{~A} \Delta t}{l} \mathrm{erg},
$$

where $\kappa$ is the Spitzer conductivity $\left(8.8 \times 10^{-7} \mathrm{erg} \mathrm{cm}^{-1} \mathrm{~s}^{-1}\right.$ $\mathrm{K}^{-7 / 2}$ ), $l$ describes the length scale of energy loss, $A$ is the area of the footpoint, and $\Delta t$ is the flare time interval. For this estimate, we assume a geometry of a cylindrical loop of length $2 l$ with footpoint radius $r$, which is related by the aspect ratio of $\alpha=\frac{r}{2 l}=0.1$ (considered an upper limit for solar flares). With these assumptions incorporated into Equation (1), the conductive losses can be computed in terms of an unknown length, $l$. For the flare on IRS 43 , the conductive energy losses are estimated as $E_{\text {cond }} \sim(4 \times$ $\left.10^{24}\right) \times l$ erg. By freezing the aspect ratio, we note that $A \propto l^{2}$ and thus the conductive losses increase with the loop length rather than being inversely proportional.

We expect that the combined energy in radiative and conductive losses should be equal to the overall energy in the thermal plasma:

$$
E_{\text {therm }}=3 k T \sqrt{E M * V},
$$

where $V$ is the volume of the heated flare plasma. Using this relationship, $E_{\text {therm }} \sim E_{\text {rad }}+E_{\text {cond }}$, and the cylindrical loop geometry described above $\left(V=\pi r^{2}(2 l)\right)$, we can solve for the loop length. Through this process, we estimate $l \sim 5 \times 10^{11} \mathrm{~cm}$, which is consistent with typical values of stellar flare loop lengths ${ }^{10}\left(\sim 10^{11}-10^{12} \mathrm{~cm}\right)$. In a case where conductive losses occur early in the flare, it may be more accurate to assume $E_{\text {therm }} \sim E_{\text {rad }}$. In this scenario, the estimated length is of the same order, with $l \sim 4 \times 10^{11} \mathrm{~cm}$. In either case, the resulting total thermal energy is of the order of $E_{\text {therm }} \sim 10^{37} \mathrm{erg}$, which is orders of magnitude smaller than the upper limits to nonthermal energy, as shown in Figure 5.

Taking this exploration a step further, we can then consider what loop length of $l$ would be required in order for conductive losses to exceed the possible energy in nonthermal electrons $\left(E_{\mathrm{NT}}\right)$. Assuming the upper limit of $E_{\mathrm{NT}} \sim 10^{41} \mathrm{erg}$, an unreasonably large loop length of $l \sim 10^{17} \mathrm{~cm}$ is required for $E_{\text {cond }}>E_{\mathrm{NT}}$. We note that this result assumes a fixed aspect ratio of $\alpha=0.1$, which is considered an upper limit for solar flares; using a smaller aspect ratio would require an even larger loop length for conductive losses to exceed energy in nonthermal electrons. This unlikely scenario further supports the case for the energy in conductive losses being much lower than our upper limits to the nonthermal electron energy. Therefore, we find that electron energy could plausibly account for both radiative and conductive losses in the flare on IRS 43.

\subsection{Iron Fluorescence and Disk Ionization}

By considering the $\mathrm{EW}$ of the $6.4 \mathrm{keV}$ emission line, we can learn more about the ionizing radiation causing fluorescence, the fluorescing material, and the geometry of the source. For IRS 43 and WL 19, we find the best estimates of the EW to be relatively large: $\sim 190 \mathrm{eV}$ and $\sim 230 \mathrm{eV}$, respectively. If we assume that fluorescence comes from photoionization of spherically distributed material around the source, the EW is

\footnotetext{
${ }^{10}$ These values for stellar flare loop lengths were estimated in Shibata \& Yokoyama (1999) using scaling relations and data from a variety of star types, including YSOs, binaries, and red dwarfs.
}

predicted to be around $\sim 10 \mathrm{eV}$, based on $N_{\mathrm{H}}$ and $Z_{\mathrm{Fe}}$ (Inoue 1985). Thus, if we utilize the best-fit EW values for our spectra, the fluorescence cannot be produced by material in the line of sight but instead requires interaction with denser material (George \& Fabian 1991; Sekimoto et al. 1997).

In the case of YSOs, a $6.4 \mathrm{keV}$ line in the flare spectrum with a large $\mathrm{EW}$ is often attributed to fluorescence in the circumstellar disk (Imanishi et al. 2001; Favata et al. 2005; Tsujimoto et al. 2005). Assuming photoionization of disk material, we would expect an EW only up to $120 \mathrm{eV}$, which is still smaller than the best-fit values, aside from that of EL 29 (George \& Fabian 1991). However, in a case where the flare is hidden behind the star's limb, the EW may be larger than $120 \mathrm{eV}$ due to attenuation of the continuum relative to the fluorescence emission from the disk (Drake et al. 2008).

Another way to account for large EWs is through fluorescence by collisional ionization, as was suggested in a study of EL 29 by Giardino et al. (2007). In addition to observing relatively large EWs $(>200 \mathrm{eV})$, this study found that a source of accelerated electrons could better explain the significant variability in EWs than changes in the thermal spectrum.

Given the large uncertainties of the EWs for our study (noted in Section 3.2.2), we refrain from proposing one specific mechanism for our flares but highlight that the potentially large EW may be consistent with a scenario where the disk serves as the main fluorescing material as opposed to the photosphere or material in the line of sight.

One important question regarding high-energy X-ray emission is how this emission impacts the process of planet formation in the protoplanetary disk (Feigelson \& Montmerle 1999; Glassgold et al. 2000; Feigelson 2010). The presence of a $6.4 \mathrm{keV}$ line and the associated EW provide evidence that X-rays from these YSO flares are plausibly interacting with their surrounding disks. X-ray emission can potentially alter the dynamics of the disk by ionizing disk material and, therefore, coupling this material to the magnetic field that, at sufficient ionization levels, could lead to MRI and eventually magnetohydrodynamical turbulence (Balbus 2011).

For a "typical" quiescent YSO $\left(k T=1 \mathrm{keV}, L \sim 10^{29} \mathrm{erg} \mathrm{s}^{-1}\right)$, work by Krolik \& Kallman (1983) estimates that ionization by stellar X-rays could dominate ionization of disk material out to $\sim 1000$ au and that much of the outer disk layer could be sufficiently ionized for MRI. During flaring times, both higher plasma temperatures and higher luminosities can lead to further penetration and an increased ionization rate (Glassgold et al. 2000). For our observed flares, we find $k T$ ranging from $3-7 \mathrm{keV}$ and average luminosities from $10^{30}-10^{31} \mathrm{erg} \mathrm{s}^{-1}$, which would correspond to broader ionization than what is found for the "typical" values. However, work by Ilgner \& Nelson (2006) indicates that the timescale for MRI is much longer than the duration of these X-ray flares, so more research is needed to determine whether transient X-ray events can lead to persistent turbulence in the disk.

\section{Summary}

During three $\sim 50 \mathrm{ks}$ observations of the $\rho$ Ophiuchi cloud complex by $N U S T A R$, multiple bright X-ray flares from YSOs were observed. NuSTAR offers unprecedented sensitivity in the hard X-ray regime above $\sim 7 \mathrm{keV}$, making this data set the first of its kind. Spectral analyses of flares on IRS 43, WL 19, and EL 29 found temperatures ranging from $\sim 40-80 \mathrm{MK}$ and emission measures of the order of $10^{54} \mathrm{~cm}^{-3}$ using an isothermal 
model. These results offer confirmation of what has been found for temperatures and brightnesses in previous X-ray surveys of flaring YSOs in the $\rho$ Ophiuchi region.

The flares presented here show no clear evidence for a higher-energy nonthermal component, but estimates through scaling laws indicate that it may be possible to observe nonthermal emission with a hotter and brighter flare in future observations. Estimates were made of thermal radiative and conductive energy along with upper limits to the energy in nonthermal electrons for the flare on IRS 43. According to our estimates, the energy in nonthermal electrons, if present, could plausibly account for both radiative and conductive losses, which is consistent with the standard model for solar and stellar flares.

Spectral analyses of the flares on IRS 43 and WL 19 suggest the presence of a $6.4 \mathrm{keV}$ emission line. In these cases, the large estimated EW may be consistent with a scenario where flare radiation interacts with dense material in the surrounding disk, producing fluorescence through photoionization. In addition to fluorescence, X-rays from flaring YSOs may significantly ionize disk material, depending on the flare luminosity, temperature, and frequency. Additional modeling and observations in the high-energy X-ray regime with $N U S T A R$ are necessary in order to further investigate the impact of these extreme stellar events.

This work was supported by NSF grants AGS-1429512 and AGS-1752268 and by NASA Headquarters under the NASA Earth and Space Science Fellowship Program Grant 80NSSC17K0430. The authors would like to acknowledge and thank Eric Feigelson for encouraging the NuSTAR team early on to make these observations and for contributing to discussions of this analysis.

Software: NuSTARDAS (v1.6.0), nuskybgd (Wik et al. 2014), XSPEC (Arnaud 1996).

\section{ORCID iDs}

Juliana T. Vievering (1D https://orcid.org/0000-0002-7407-6740 Lindsay Glesener (D) https://orcid.org/0000-0001-7092-2703

Brian W. Grefenstette (iD https://orcid.org/0000-00021984-2932
David M. Smith (10 https://orcid.org/0000-0002-0542-5759

\section{References}

Anders, E., \& Grevesse, N. 1989, GeCoA, 53, 197

Arnaud, K. A. 1996, adass V, 101, 17

Aschwanden, M. J., Holman, G., O’Flannagain, A., et al. 2016, ApJ, 832, 27

Audard, M., Güdel, M., \& Guinan, E. F. 1999, ApJL, 513, L53

Balbus, S. A. 2011, in Physical Processes in Circumstellar Disks around Young Stars, ed. J. V. Garcia (Chicago, IL: Univ. Chicago Press), 232

Battaglia, M., Grigis, P. C., \& Benz, A. O. 2005, A\&A, 439, 737

Brown, J. C. 1971, SoPh, 18, 489

Drake, J. J., Ercolano, B., \& Swartz, D. A. 2008, ApJ, 678, 385

Elias, J. H. 1978, ApJ, 224, 453

Favata, F., Micela, G., Silva, B., Sciortino, S., \& Tsujimoto, M. 2005, A\&A, 433, 1047

Feigelson, E. D. 2010, PNAS, 107, 7153

Feigelson, E. D., \& Montmerle, T. 1999, ARA\&A, 37, 363

George, I. M., \& Fabian, A. C. 1991, MNRAS, 249, 352

Giardino, G., Favata, F., Pillitteri, I., et al. 2007, A\&A, 475, 891

Glassgold, A. E., Feigelson, E. D., \& Montmerle, T. 2000, in Protostars and Planets IV, ed. V. Mannings, A. P. Boss, \& S. S. Russell (Tucson, AZ: Univ. Arizona Press), 429

Harrison, F. A., Craig, W. W., Christensen, F. E., et al. 2013, ApJ, 770, 103 Ilgner, M., \& Nelson, R. P. 2006, A\&A, 455, 731

Imanishi, K., Nakajima, H., Tsujimoto, M., Koyama, K., \& Tsuboi, Y. 2003, PASJ, 55, 653

Imanishi, K., Tsujimoto, M., \& Koyama, K. 2001, ApJ, 563, 361

Inoue, H. 1985, SSRv, 40, 317

Isola, C., Favata, F., Micela, G., \& Hudson, H. S. 2007, A\&A, 472, 261

Kane, S. R., McTiernan, J. M., \& Hurley, K. 2005, A\&A, 433, 1133

Krolik, J. H., \& Kallman, T. R. 1983, ApJ, 267, 610

Lada, C. J., \& Wilking, B. A. 1984, ApJ, 287, 610

Loinard, L., Torres, R. M., Mioduszewski, A. J., \& Rodríguez, L. F. 2008, ApJL, 675, L29

Maggio, A., Flaccomio, E., Favata, F., et al. 2007, ApJ, 660, 1462

Neupert, W. M. 1968, ApJL, 153, L59

Osten, R. A., Drake, S., Tueller, J., et al. 2007, ApJ, 654, 1052

Pillitteri, I., Sciortino, S., Flaccomio, E., et al. 2010, A\&A, 519, A34

Sekimoto, Y., Tatematsu, K., Umemoto, T., et al. 1997, ApJL, 489, L63

Shibata, K., \& Yokoyama, T. 1999, ApJL, 526, L49

Tsujimoto, M., Feigelson, E. D., Grosso, N., et al. 2005, ApJS, 160, 503

Veronig, A., Vršnak, B., Dennis, B. R., et al. 2002, A\&A, 392, 699

Warmuth, A., \& Mann, G. 2016, A\&A, 588, A116

Wik, D. R., Hornstrup, A., Molendi, S., et al. 2014, ApJ, 792, 48

Wilking, B. A., \& Lada, C. J. 1983, ApJ, 274, 698

Wilking, B. A., Lada, C. J., \& Young, E. T. 1989, ApJ, 340, 823

Woods, T. N., Eparvier, F. G., Fontenla, J., et al. 2004, GeoRL, 31, L10802

Woods, T. N., Kopp, G., \& Chamberlin, P. C. 2006, JGRA, 111, A10S14 